\title{
T cells targeted to TdT kill leukemic lymphoblasts while sparing normal lymphocytes
}

\author{
Muhammad Ali ${ }^{1,2,22}$, Eirini Giannakopoulou ${ }^{1,2,22}$, Yingqian $\mathrm{Li}^{1,2}$, Madeleine Lehander ${ }^{3}$, \\ Stina Virding Culleton ${ }^{3}$, Weiwen Yang ${ }^{1,2}$, Cathrine Knetter ${ }^{1,2}$, Mete Can Odabasi ${ }^{4}$, \\ Ravi Chand Bollineni ${ }^{1,2}$, Xinbo Yang ${ }^{5}$, Zsofia Foldvari ${ }^{1,2}$, Maxi-Lu Böschen ${ }^{1,2}$, Eli Taraldsrud ${ }^{1,2}$, \\ Erlend Strønen ${ }^{1,2}{ }^{1}$, Mireille Toebes ${ }^{6}$, Amy Hillen ${ }^{19}$, Stefania Mazzi ${ }^{3}$, Arnoud H. de Ru ${ }^{7}$, \\ George M. C. Janssen ${ }^{7}$, Arne Kolstad ${ }^{2,8}$, Geir Erland Tjønnfjord ${ }^{9}$, Benedicte A. Lie ${ }^{10}$, \\ Marieke Griffioen (iD ${ }^{11}$, Sören Lehmann ${ }^{3,12}$, Liv Toril Osnes ${ }^{13}$, Jochen Buechner ${ }^{14}$,

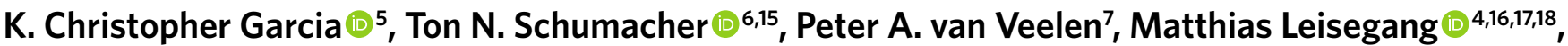 \\ Sten Eirik W. Jacobsen $3,19,20,21,23$, Petter Woll $\mathbb{1}^{3,23}$ and Johanna Olweus ${ }^{1,2} \bowtie$
}

\begin{abstract}
Unlike chimeric antigen receptors, T-cell receptors (TCRs) can recognize intracellular targets presented on human leukocyte antigen (HLA) molecules. Here we demonstrate that T cells expressing TCRs specific for peptides from the intracellular lymphoid-specific enzyme terminal deoxynucleotidyl transferase (TdT), presented in the context of HLA-A ${ }^{\star} 02: 01$, specifically eliminate primary acute lymphoblastic leukemia (ALL) cells of T- and B-cell origin in vitro and in three mouse models of disseminated B-ALL. By contrast, the treatment spares normal peripheral T- and B-cell repertoires and normal myeloid cells in vitro, and in vivo in humanized mice. TdT is an attractive cancer target as it is highly and homogeneously expressed in $80-94 \%$ of B- and T-ALLs, but only transiently expressed during normal lymphoid differentiation, limiting on-target toxicity of TdT-specific T cells. TCR-modified T cells targeting TdT may be a promising immunotherapy for B-ALL and T-ALL that preserves normal lymphocytes.
\end{abstract}

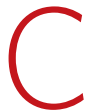
D19 chimeric antigen receptor (CAR) T-cell therapy frequently induces complete remission in acute B-lymphoblastic leukemia (B-ALL), dramatically improving the outcome for this patient group. However, approximately $40-50 \%$ of patients relapse $^{1,2}$, most frequently due to loss of CD19 (refs. ${ }^{3-5}$ ). CAR T-cell therapy directed at other B-cell-specific cell-surface molecules has shown a higher tendency to relapse ${ }^{6}$, possibly because of lower expression levels than CD19 on leukemia-propagating cells. Cell-based immunotherapies targeting alternative molecules expressed early during B-cell differentiation would thus be desirable. In spite of a multitude of T-cell-specific markers, no CAR therapy is approved for T-ALL that efficiently targets malignant cells across all T-ALL subtypes while at the same time sparing mature, normal T cells. This would be crucial to avoid depletion of normal
T cells, which is prohibitively toxic or even incompatible with life Moreover, no tumor-specific target has been identified in T-ALL and no immunotherapy has yet proven effective in clinical trials. In the event of failed chemotherapy (15-20\%), T-ALL has a dismal prognosis with overall survival $<25 \%$ (refs. ${ }^{7,8}$ ).

We hypothesized that the enzyme terminal deoxynucleotidyl transferase (TdT) might be an ideal target of immunotherapy in B-ALL and T-ALL, for several reasons. First, as the function of TdT is to add N-nucleotides to the V-D-J junctions during recombination of the TCR and B-cell receptor ${ }^{9}$, its expression is confined to the $\mathrm{B}$ and $\mathrm{T}$ lineages. Second, TdT is overexpressed in 80-94\% of ALL and lymphoblastic lymphoma of B- and T-cell origin $^{10-12}$, making it a widely used diagnostic marker ${ }^{13}$. Third, expression of $\mathrm{TdT}$ is restricted to a narrow window during

\footnotetext{
'Department of Cancer Immunology, Institute for Cancer Research, Oslo University Hospital Radiumhospitalet, Oslo, Norway. ${ }^{2}$ K.G. Jebsen Center for Cancer Immunotherapy, Institute of Clinical Medicine, University of Oslo, Oslo, Norway. ${ }^{3}$ Department of Medicine, Huddinge Center for Hematology and Regenerative Medicine, Karolinska Institutet, Stockholm, Sweden. ${ }^{4}$ Charité - Universitätsmedizin Berlin, Institute of Immunology, Berlin, Germany. ${ }_{5}^{5}$ Parker Institute for Cancer Immunotherapy, Howard Hughes Medical Institute, Departments of Molecular and Cellular Physiology and Structural Biology, Stanford University School of Medicine, Stanford, CA, USA. ${ }^{6}$ Division of Molecular Oncology \& Immunology, Oncode Institute, The Netherlands Cancer Institute, Amsterdam, the Netherlands. ${ }^{7}$ Center for Proteomics and Metabolomics, Leiden University Medical Center, Leiden, the Netherlands. ${ }^{8}$ Department of Oncology, Oslo University Hospital Radiumhospitalet, Oslo, Norway. ${ }^{9}$ Department of Haematology, Oslo University Hospital and KG Jebsen Center for B cell malignancies, Institute of Clinical Medicine, University of Oslo, Oslo, Norway. ${ }^{10}$ Department of Medical Genetics, University of Oslo and Oslo University Hospital, Oslo, Norway. "'Department of Hematology, Leiden University Medical Center, Leiden, the Netherlands. ${ }^{12}$ Department of Medical Sciences, Uppsala University Hospital, Uppsala, Sweden. ${ }^{13}$ Department of Immunology, Oslo University Hospital, Oslo, Norway. ${ }^{14}$ Department of Pediatric Hematology and Oncology, Oslo University Hospital, Oslo, Norway. ${ }^{15}$ Department of Immunohematology and Bloodtransfusion, Leiden University Medical Center, Leiden, the Netherlands. ${ }^{16}$ David and Etta Jonas Center for Cellular Therapy, The University of Chicago, Chicago, IL, USA. ${ }^{17}$ German Cancer Consortium, partner site Berlin, Berlin, Germany. ${ }^{18} \mathrm{German}$ Cancer Research Center, Heidelberg, Germany. ${ }^{19}$ Department of Cell and Molecular Biology, Karolinska Institutet, Stockholm, Sweden. ${ }^{20}$ Karolinska University Hospital, Stockholm, Sweden. ${ }^{21}$ MRC Molecular Haematology Unit, MRC Weatherall Institute of Molecular Medicine, University of Oxford, Oxford, UK. ${ }^{22}$ These authors contributed equally: Muhammad Ali, Eirini Giannakopoulou.

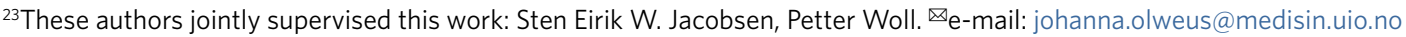




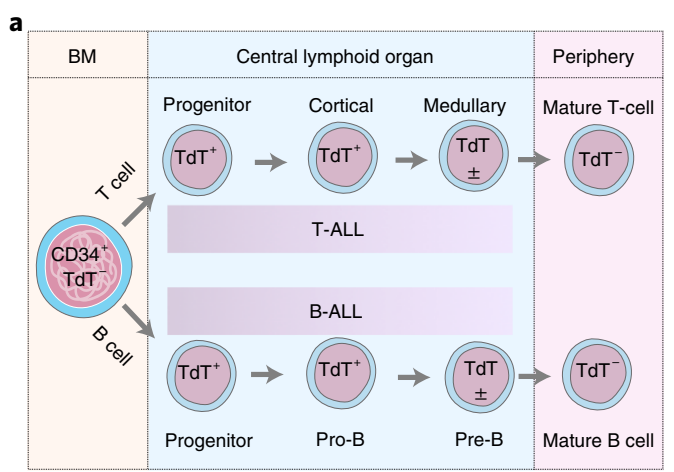

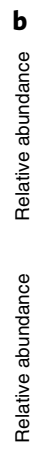
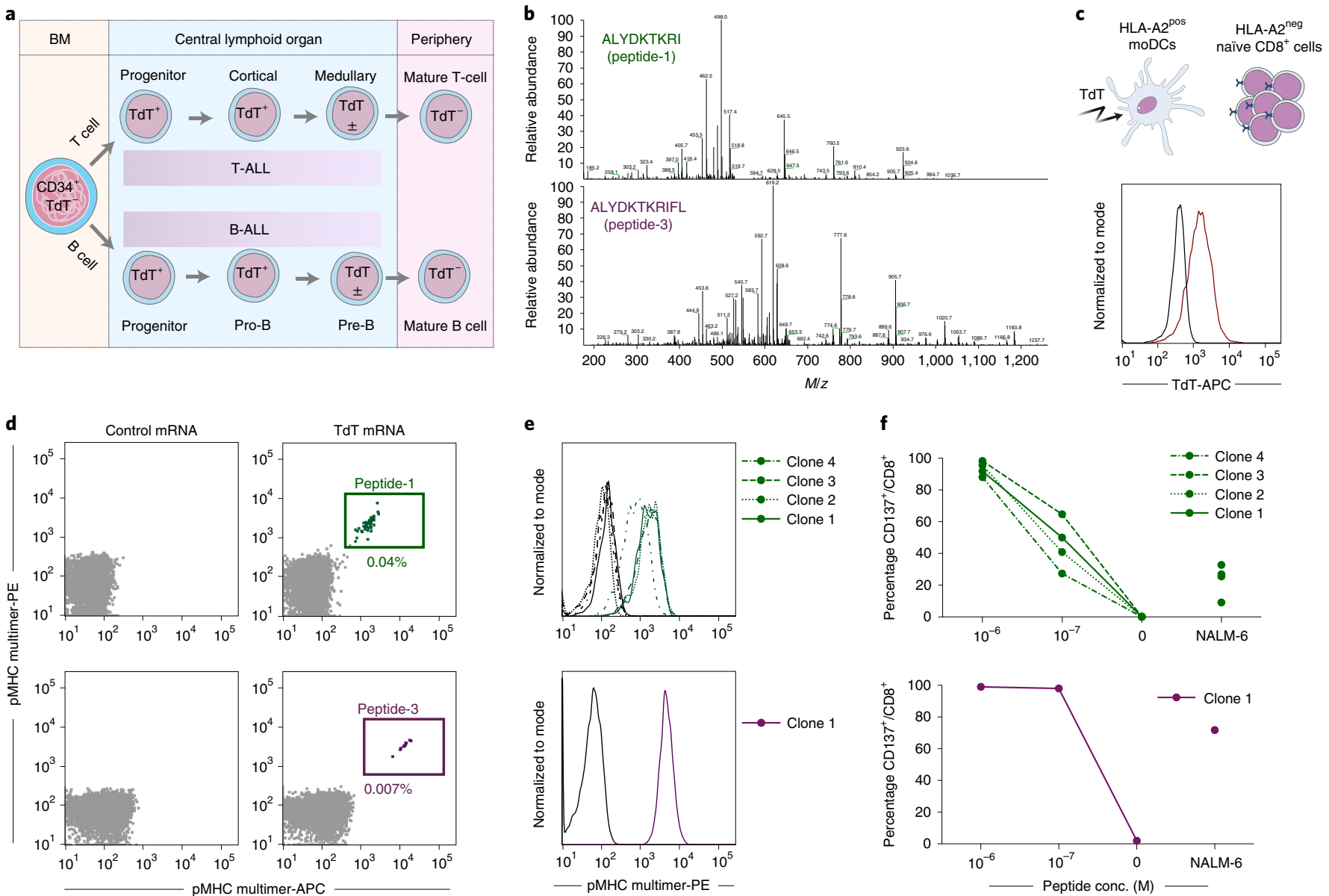

Fig. 1 | Discovery of CD8 ${ }^{+}$T-cell clones reactive to TdT peptides presented on HLA-A2. a, lllustration of TdT expression during lymphoid differentiation. b. Mass to charge $(\mathrm{m} / \mathrm{z})$ ratio spectra of TdT peptide-1 and -3 . c, Intracellular staining of TdT in HLA-A2 ${ }^{\text {pos }}$ moDCs after electroporation with mRNA encoding full-length TdT (red) or irrelevant control mRNA (black). HLA-A2 $2^{\text {pos }}$ moDCs were then cocultured with HLA-A2 $2^{\text {neg }}$ naïve CD8 ${ }^{+}$cells. d, Staining of $\mathrm{CD}^{+}$T cells with $\mathrm{pMHC}$ multimers complexed with peptide-1 or -3 (each multimer conjugated to both APC and PE, gating strategy shown in Extended Data Fig. 1a) following cocultures with moDCs transfected with TdT or control mRNA. e, Staining of T-cell clones reactive to peptide-1 (clones 1-4) and peptide-3 (clone 1) showing the relevant pMHC multimers (green and purple) and corresponding nonrelevant pMHC multimers (black). f, Upregulation of CD137 on T-cell clones reactive to peptide-1 (green, clones 1-4) and peptide-3 (purple, clone 1) following coincubation with TdT Thes HLA-A2 ${ }^{\text {pos }}$ EBV-LCL cells pulsed with indicated concentrations of cognate peptides, or the B-ALL cell line NALM-6, naturally positive for TdT and HLA-A2. conc., concentration.

differentiation of early B- and T-cell progenitors in bone marrow (BM) and thymus, and it is not expressed in hematopoietic stem cells ${ }^{14}$. Therefore, it is expected that both naïve and mature $\mathrm{B}$ and Tlymphocytes, as well as myeloid cells, would be spared ${ }^{15}$ (Fig. 1a). However, since TdT is localized intracellularly it cannot be targeted by a CAR. In contrast to CARs, TCRs can recognize intracellular antigens presented on the cell surface in complex with major histocompatibility complex (MHC) molecules. T cells that bind self-antigens with high affinity are, however, depleted from the autologous repertoire during thymic negative selection. To circumvent this, we used a technology whereby our previously published protocol for identification of healthy donor Tcells that recognize foreign neoantigens presented on self-HLA ${ }^{16}$ was modified to allow for selection of high-affinity T cells reactive to self-antigen presented in the context of foreign HLA. We identified TCRs recognizing TdT-derived peptides presented on HLA-A ${ }^{\star} 02: 01$ (HLA-A2), expressed in approximately $50 \%$ of people of European, Middle Eastern or North African ancestry. We also evaluated the safety and efficacy of these TCRs following treatment of leukemia with TCR-engineered T cells in vitro and in vivo.

\section{Results}

We determined the sequence of TdT-derived peptides that were naturally processed and presented on HLA-A2 from HLA-A2 $2^{\text {pos }}$ Epstein-Barr virus-transformed lymphoblastoid cells (EBV-LCL) transduced with full-length TdT, by mass spectrometry (MS). Two peptides identified as HLA-A2 binders (ALYDKTKRI (peptide-1) and ALYDKTKRIFL (peptide-3)) were further characterized (Fig. 1b, Supplementary Fig. 1a,b and Supplementary Table 1). Naïve T cells from HLA-A2 ${ }^{\text {neg }}$ donors were cocultured with monocyte-derived dendritic cells (MoDCs) generated from HLA-A2 $2^{\text {pos }}$ donors that were electroporated with TdT-encoding messenger RNA (Fig. $1 c)^{16}$. T cells staining positively with peptide-major histocompatibility complex (pMHC)-multimers (Fig. 1d) were sorted as single cells to generate T-cell clones. Clones reactive to peptide- 1 and peptide-3 stained positively with the relevant multimers (Fig. 1e) and were activated by peptide-pulsed HLA-A2 ${ }^{\text {pos }}$ EBV-LCL, and by a B-ALL cell line endogenously expressing TdT (Fig. 1f). One TCR sequence was identified from clones reactive to each peptide, named T1 (reactive to peptide 1) and T3 (reactive to peptide-3). Both TCRs were efficiently expressed in third-party peripheral blood (PB) $\mathrm{CD}^{+} \mathrm{T}$ cells, as demonstrated by staining with either 
pMHC-multimers or anti-mouse TCR- $\beta$ antibodies reactive to the mouse constant region introduced into the TCRs ${ }^{17}$ (Fig. 2a). All T3-transduced cells and the majority of T1-transduced cells that stained positively with anti-mouse TCR- $\beta$ were also pMHC-multimer positive, indicating preferential pairing of introduced TCR- $\alpha$ and $-\beta$ chains after retroviral transduction (Extended Data Fig. 1a) ${ }^{18}$. $\mathrm{T}$ cells from HLA-A2 $2^{\text {pos }}$ donors that were transduced with $\mathrm{T} 1$ and $\mathrm{T} 3$ ( $\mathrm{T} 1$ and $\mathrm{T} 3$ cells) expanded equally well as those transduced with the control TCR 1G4 (Extended Data Fig. 1b), specific for NY-ESO-1 and safely used in clinical trials ${ }^{19}$. The results indicate the absence of fratricide, as expected from lack of TdT expression in PB T cells ${ }^{15}$. The majority of transduced cells had a naïve or central memory phenotype (Extended Data Fig. 1c). T1 and T3 cells recognized their cognate peptides with high sensitivity (T1 half-maximal effective concentration $\left(\mathrm{EC}_{50}\right)=5.8 \mathrm{nM}$ and $\mathrm{T} 3 \mathrm{EC}_{50}=1.2 \mathrm{nM}$; Fig. $2 \mathrm{~b}$ ).

T1 and T3 cells recognized target cells in an HLA-A2-restricted and TdT-dependent manner. Thus, T1 and T3 cells were activated only by EBV-LCLs presenting TdT peptides introduced by either mRNA electroporation or external peptide loading when HLA-A2 was simultaneously expressed (Fig. 2c). Similarly, naturally $\mathrm{TdT}^{\mathrm{pos}}$ but HLA-A2 ${ }^{\text {neg }}$ cell lines REH (B-ALL origin) and HPB-ALL (T-ALL origin) activated T1 and T3 cells only when HLA-A ${ }^{\star} 02: 01$ was introduced. Activation was reduced by the addition of the MHC class I blocking antibody W6/32 (Fig. 2d). Moreover, CRISPRCas9-mediated knockout of TdT abolished recognition of the B-ALL cell line NALM-6 (naturally TdT ${ }^{\text {pos }}$ and HLA-A2 ${ }^{\text {pos }}$ ) by T1 and T3 cells (Fig. 2e and Extended Data Fig. 1d,e). T-cell activation was not observed when T1 and T3 cells were cocultured with a large panel of human $\mathrm{TdT}^{\text {heg }}$ cell lines of different tissue origin, unless loaded with relevant peptide and expressing HLA-A2, naturally or by genetic introduction (Fig. 2f). These results showed that T1 and T3 TCRs did not react with unintended targets presented on HLA-A2, or on a large variety of other HLA molecules expressed by the cell lines.

Precise mapping of TCR reactivity can inform about cross-reactivity of potential clinical importance. To this end, we synthesized peptide mimotope libraries in which each amino acid residue of peptide- 1 or -3 was replaced by all other natural amino acids, one at a time. T1 and T3 cells were combined with target cells loaded with single mimotopes from the relevant library. Resultant T-cell activation, as measured by production of IFN- $\gamma$ or upregulation of CD137, was highly correlated (Fig. $2 \mathrm{~g}$ and Extended Data Fig. 2a-c). Next, we queried the curated the human proteome databases UniProtKB/Swiss-Prot and Protein Data Bank for all combinations of amino acid substitutions that induced reactivity in T1 or T3 cells, by employing the ScanProsite tool (https:// prosite.expasy.org/scanprosite/) (Extended Data Fig. 2d). The search did not identify any naturally occurring 9- or 11-mer peptide in the human proteome that matched these combinations. When searching a noncurated database (UniProtKB/TrEMBL), one peptide derived from the ubiquitously expressed small integral membrane protein 19 matched reactivity combinations for T3 but did not induce any response in T3 cells (Extended Data Fig. 2e,f). Taken together, the data provided no evidence for off-target reactivities by $\mathrm{T} 1$ and T3. Moreover, peptides prolonged upstream and/ or downstream of peptide- 1 and -3 sequences in the TdT protein failed to activate T1 and T3 cells, unless longer variants encompassing the whole cognate peptide sequence were used (Extended Data Fig. 3a,b). Furthermore, peptide-1 failed to activate T3 cells whereas peptide- 3 activated T1 cells only at high concentrations, probably due to the breakdown of peptide-3 generating peptide-1 in culture (Extended Data Fig. 3c). Lack of cross-activation might be explained by a large difference in the structural configuration of the two peptide-MHC complexes modeled in Fig. $2 \mathrm{~h}$ and Extended Data Fig. 3d. Peptide-1 adopts a flattened conformation in the HLA-A2 peptide groove whereas peptide-3 displays a bulging conformation. To accommodate this, T3 is likely to be 'lifted up' and make more interactions with peptide- 3 but less contact with MHC, while T1 makes more evenly distributed interactions with peptide- 1 and MHC.

T1 and T3 cells responded strongly to B-cell leukemia cell lines BV173 and NALM-6 (naturally TdT ${ }^{\text {pos }}$ and HLA-A2 ${ }^{\text {pos }}$ ), as measured by the production of IFN- $\gamma$, proliferation and killing (96-99\% at an effector/target (E/T) ratio of 1/1) (Fig. 3a and Extended Data Fig. $4 \mathrm{a}-\mathrm{g}$ ). To study the efficacy in vivo, we engrafted BV173 ffluc-eGFP cells or NALM-6 ffluc-eGFP cells in NOD-scid IL2Rg ${ }^{\text {null }}$ (NSG) mice and started treatment with T1 or T3 cells after leukemia establishment (Fig. $3 \mathrm{~b}$ and Extended Data Figs. 5a-d and 6a,d). A very low (T1) or no (T3) tumor signal was observed in these mice on days 21 (BV173; Fig. 3c,d) and 14 (NALM-6, Fig. 3g,h), shortly after which untreated and 1G4-treated control mice had to be sacrificed because of high tumor burden (Fig. 3e,i). Notably, none of the mice treated with T3 cells died from leukemia during the observation period following injection of leukemic cells (Fig. 3e,i). Two T3 cell-treated mice in the NALM-6 model died from reasons unknown and not related to leukemia spread. Bioluminescence imaging (BLI) remained negative on day 57 in the T3 cell-treated mice in the NALM- 6 model, consistent with absence of tumor in the BM of sacrificed mice (Extended Data Fig. 6e-g). Only one out of five T3-treated BV173 mice had green fluorescent protein $(\mathrm{GFP})^{+}$tumor cells in the BM upon sacrifice, probably due to reduced T-cell numbers and not related to antigen downregulation (Extended Data Fig. 5e-g). Mice treated with T3 cells had higher IFN- $\gamma$ levels in serum on day 2 after adoptive T-cell transfer as compared with those treated with 1G4 T cells (Extended Data Fig. 6h). TCR-transduced Tcells were still detected in peripheral blood at the experimental endpoint (Fig. 3f,j and Extended Data Figs. 5b-d and $6 \mathrm{~b}-\mathrm{d}$ ), although their numbers decreased after tumor eradication. Control 1G4 TCR-transduced T cells also expanded initially, probably due to injections of IL-2. Survival benefit was highly significant also for BV173 mice treated with T1 cells (Fig. 3e), but several of these mice eventually succumbed to tumor, consistent with the lower peptide sensitivity for $\mathrm{T} 1$ as compared with $\mathrm{T} 3$.

We next quantified the ability of T1 and T3 cells to selectively recognize human primary ALL cells in samples also containing normal $\mathrm{B}$ and Tcells and nonlineage-committed hematopoietic progenitor cells. Cryopreserved $\mathrm{TdT}^{\mathrm{pos}}$ and HLA-A2 ${ }^{\text {pos }}$ diagnostic samples from nine patients with B-ALL and three with T-ALL (Extended Data Fig. 7a and Supplementary Table 2) were cocultured with T1 or T3 cells. Following 48-72 h of coculture, T3 cells eliminated on average $97 \%$, and T1 cells $69 \%$, of leukemic blasts (T3, mean 97\%, range 92-99.9\%; T1, mean 69\%, range $13-96 \%, n=12$ ). In contrast, normal B and T cells remained unaffected (Fig. 4a,b) as did noncancerous $\mathrm{CD}_{3} 4^{+} \mathrm{lin}^{-}$cells detected in four patients (Fig. $4 a, c)$. Moreover, T1 and T3 cells were activated only by HLA-A2 and TdT double-positive patient leukemia cells. HLA-A2 ${ }^{\text {neg }} \mathrm{TdT} \mathrm{T}^{\mathrm{pos}}$ malignancies failed to activate T1 and T3 cells, even in the presence of exogenously loaded peptide, while $\mathrm{TdT}^{\text {heg }} \mathrm{HLA}-\mathrm{A} 2^{\text {pos }}$ primary follicular lymphoma and T-ALL cells activated T1 and T3 cells only when loaded with TdT peptides (Extended Data Fig. 7b). We next showed that the introduction of $\mathrm{T} 3$ into normal $\mathrm{T}$ cells from a patient with HLA-A2 ${ }^{\text {pos }}$ TdT ${ }^{\text {pos }}$ B-ALL resulted in T-cell activation and elimination of virtually all autologous tumor cells. Normal B, T and $\mathrm{CD}_{34}{ }^{+} \mathrm{lin}^{-}$progenitor cells were spared (Fig. $4 \mathrm{~d}$ and Extended Data Fig. 8). To demonstrate directly that the results above reflect the presentation of peptides- 1 and -3 on patient-derived leukemia cells, we eluted peptides from HLA-A2 molecules of patient $119 \mathrm{~N}$, as well as from BV173 cells, and identified the sequences by MS (Fig. 4e and Supplementary Fig. 2). Taken together, these data indicate a high degree of target selectivity and therapeutic efficacy of T1 and T3 cells against patient-derived B- and T-ALL cells with representative TdT and HLA-A2 expression. 
a

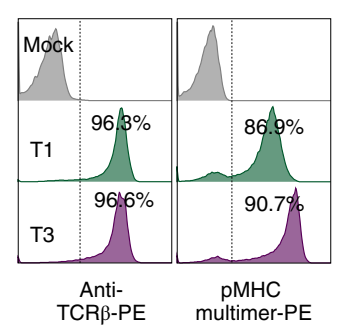

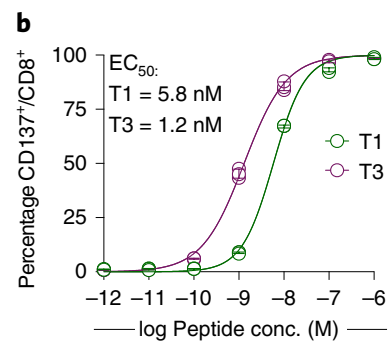

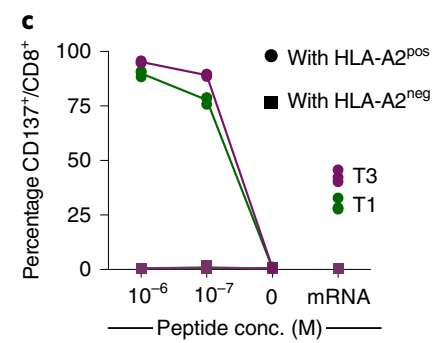

d
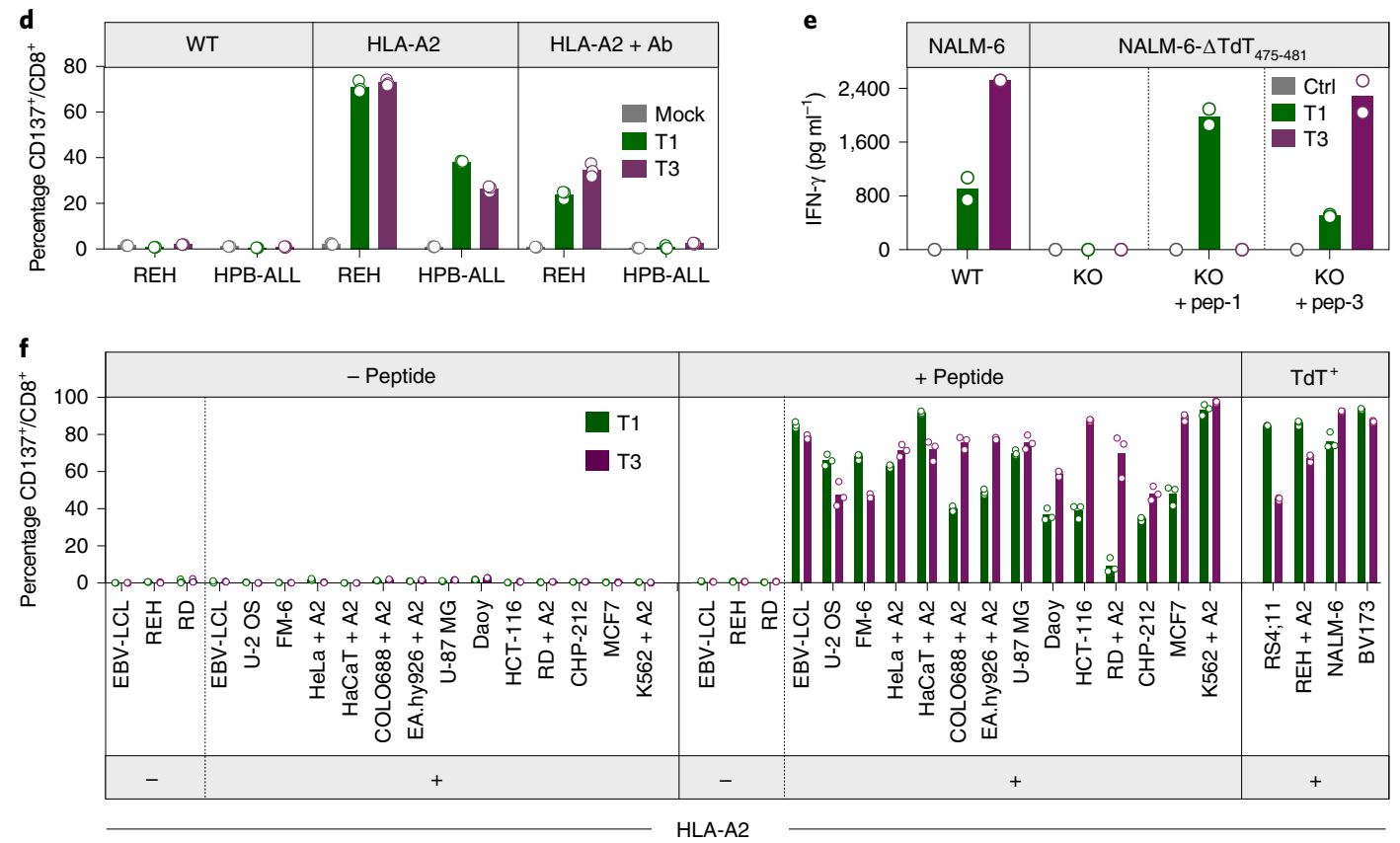

g

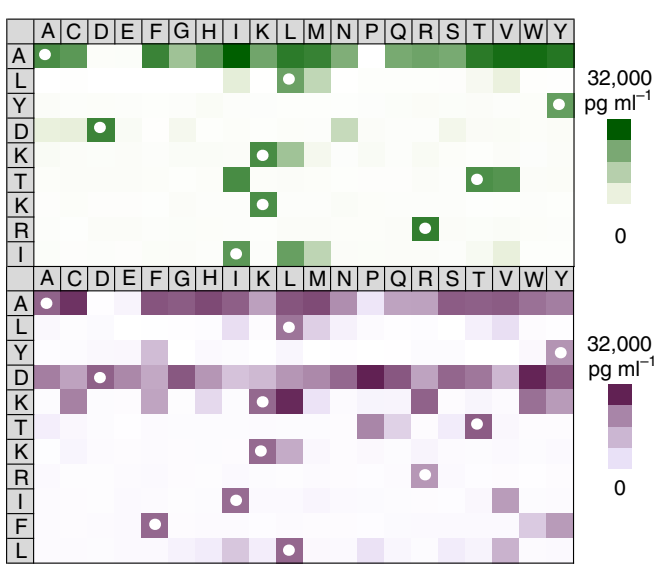

h
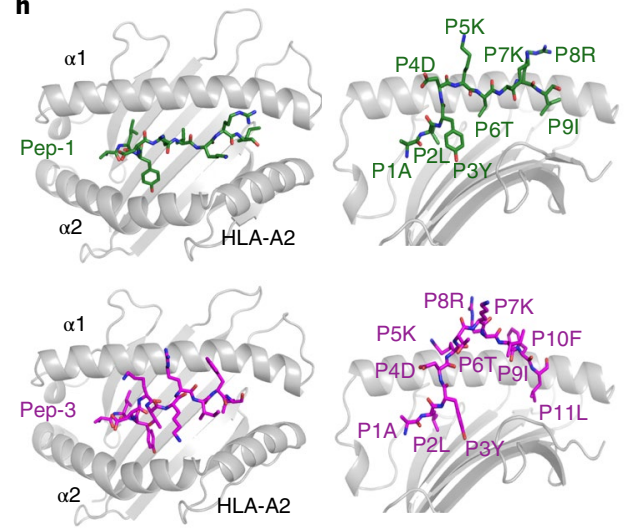

Fig. 2 | T1 and T3 are specific for TdT and HLA-A2 and do not show off-target reactivity. a, Histograms of viable CD8 ${ }^{+} T$ cells transduced with $\mathrm{T} 1$ and T3 TCRs stained with anti-mouse TCR- $\beta$ antibody or relevant peptide-MHC multimers. b. Activation of T1 and T3 cells following coincubation with peptide-pulsed T2 lymphoblast cells. Data are pooled from three independent experiments, with each circle representing the mean of three technical replicates in each experiment. Data are shown as mean \pm s.d. c, CD137 upregulation on $\mathrm{CD}^{+} \mathrm{T} 1$ (green) and T3 (purple) cells after coculture with EBV-LCLs derived from one HLA-A2 $2^{\text {pos }}$ and one HLA-A2 $2^{\text {neg }}$ donor, either pulsed with relevant peptides or electroporated with mRNA encoding full-length TdT. d, Activation of CD8 $8^{+} \mathrm{T} 1$ and T3 cells after coculture with TdToos cell lines REH (B-ALL) and HPB-ALL (T-ALL) in the presence/absence of introduced expression of HLA-A2, or pan-MHC class I blocking antibody W6/32 (Ab). e, IFN- $\gamma$ production by T1 and T3 cells following coincubation with TdTpos NALM- 6 cells (WT), or NALM- 6 cells in which TdT $475-481$ was deleted by CRISPR-Cas9 (KO) in the presence/absence of added peptide. f, Activation of T1 and T3 cells after coculture with various cell lines, with indicated HLA-A2 and TdT expression, loaded or not with TdT peptides $\left(2 \times 10^{-7} \mathrm{M}\right)$. The suffix + A2 denotes cell lines transduced with HLA-A*02:01. $\mathbf{c}-\mathbf{f}$, Results are from one experiment representative of two or three performed with different T-cell donors. Bars or connecting lines show mean, and individual data points represent either two $(\mathbf{e})$ or three $(\mathbf{c}, \mathbf{d}, \mathbf{f})$ technical replicates. $\mathbf{g}$, Heat maps of IFN- $\gamma$ produced by T1 (green) and T3 cells (purple) coincubated with EBV-LCLs pulsed with peptides from mimotope libraries. White circles, amino acid in wild-type peptide. IFN- $\gamma$ concentration range for positive reactions was 500-31,254 $\mathrm{g} \mathrm{mll}^{-1}$. One replicate per condition (see Extended Data Fig. $2 c$ for correlation with CD137 activation assay). h, Model structures of TdT peptide-1 (green) and -3 (purple) represented as sticks, bound to the HLA-A2 molecule, in gray, shown from top (left) and side views (right). Individual amino acids are labeled with positional number and symbol. 


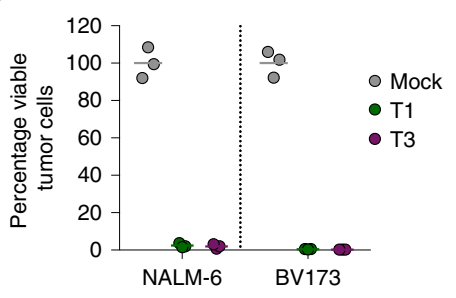

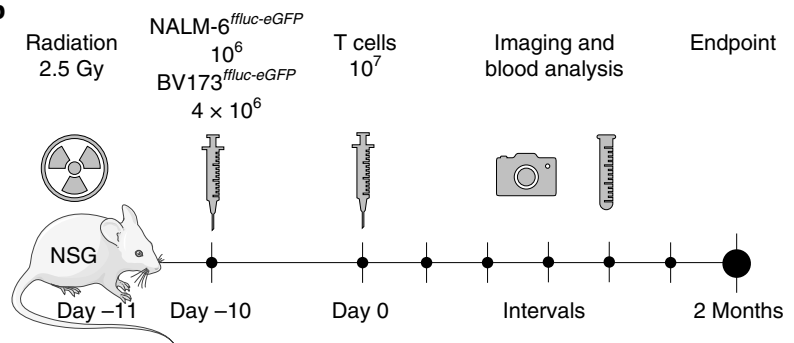

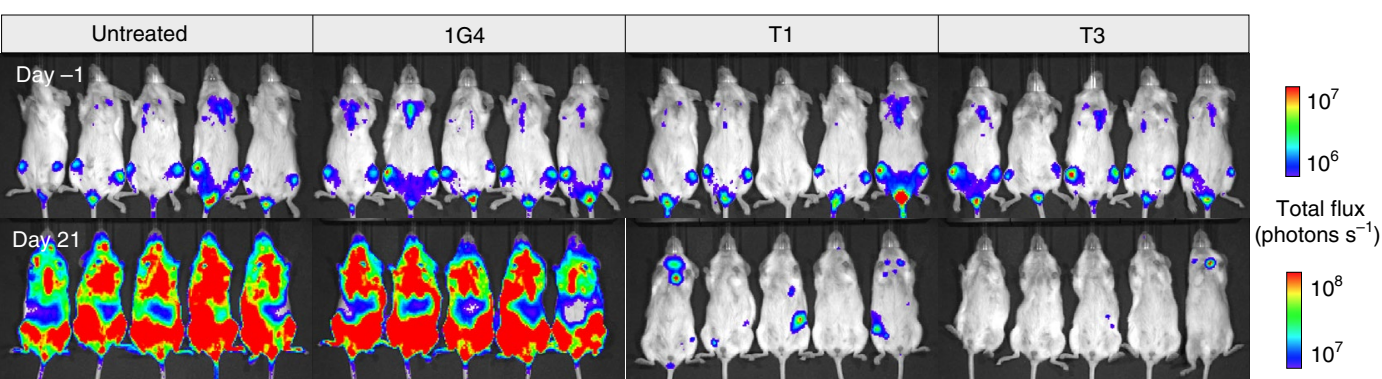

d

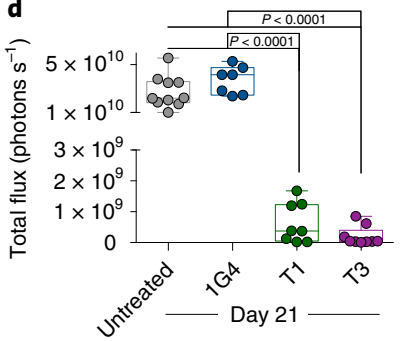

e

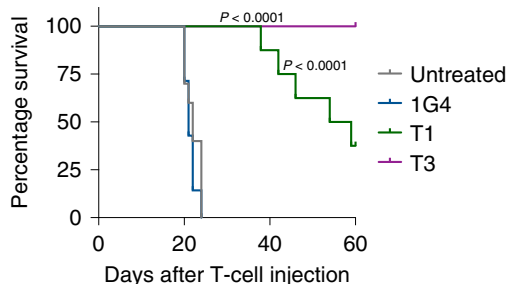

f

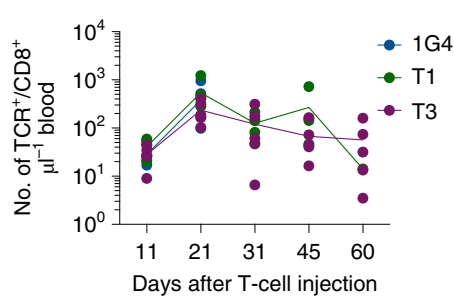

g

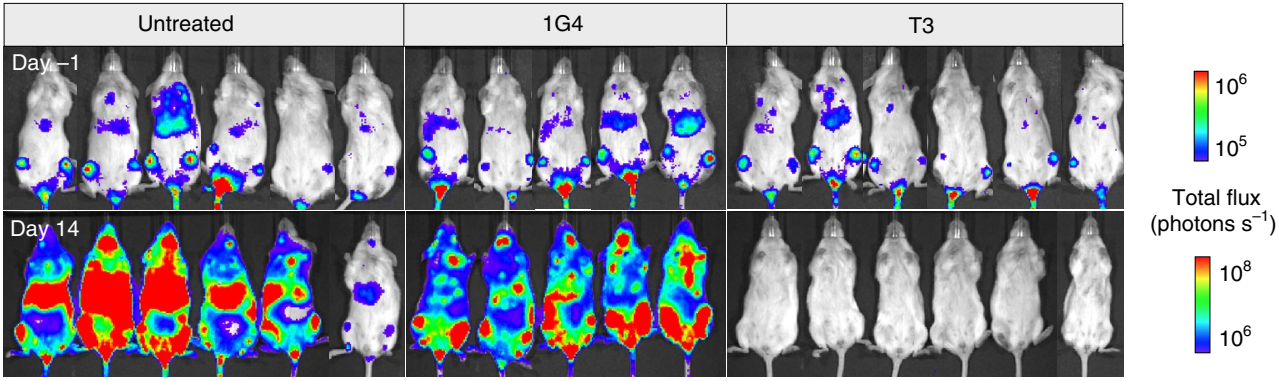

h

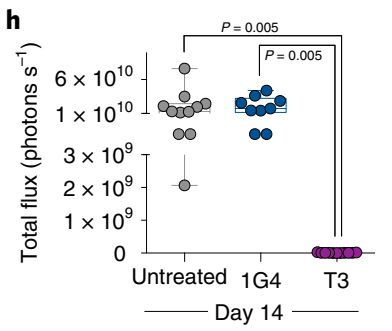

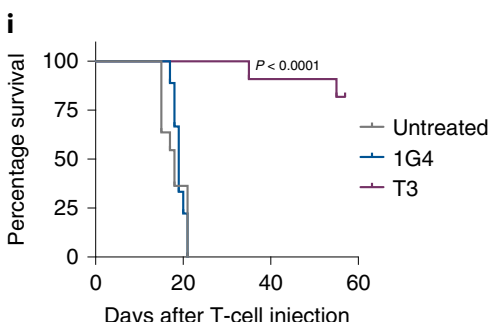

j

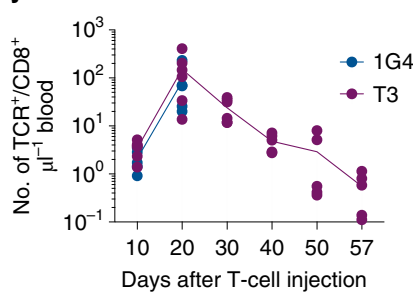

Fig. 3 | T1 and T3 cells efficiently kill leukemia cells in vivo. a, Viable TdT ${ }^{\text {pos HLA-A2 }}{ }^{\text {pos }}$ NALM-6 and BV173 cells after 48 h of coculture with T1 and T3 cells (E/T ratio, 1/1), in percentage of corresponding numbers following treatment with mock-transduced T cells as quantified by flow cytometry. Data points represent technical replicates in one experiment representative of three performed. b, Experimental overview of BV173 and NALM-6 in vivo models. BLI (c) and quantification (d) of BV173-bearing mice 1 day before and 21 days after treatment with human T cells transduced with 1G4, T1 or T3, or left untreated. Survival analysis (e) and numbers (f) of TCR-transduced CD8 ${ }^{+}$T cells $\mu l^{-1}$ blood at indicated time points in the aforementioned experimental groups. BLI (g) and quantification (h) of NALM-6-bearing mice 1 day before and 14 days after treatment with human T cells transduced with 1G4, T3, or left untreated. Survival analysis (i) and numbers ( $\mathbf{j}$ ) of TCR-transduced $\mathrm{CD} 8^{+} \mathrm{T}$ cells $\mu \mathrm{l}^{-1}$ blood at indicated time points in the aforementioned experimental groups.

$\mathbf{d}, \mathbf{e}, \mathbf{h}, \mathbf{i}$, Data are pooled from two independent experiments: untreated, $n=10$ (BV173) or $n=11$ (NALM-6); 1G4, $n=7$ (BV173) or $n=9$ (NALM-6); T1, $n=8$ (BV173); T3, $n=9$ (BV173) or $n=11$ (NALM-6). d,h, Box plots showing interquartile range (25th-75th percentile) with central bar indicating the median and whiskers indicating the range. Dots represent individual mice and data analyzed by one-way ANOVA with adjustment for multiple comparisons with Tukey's post-test. e,i, Survival analysis performed by two-sided log-rank (Mantel-Cox) test. $\mathbf{f}, \mathbf{j}$, Data shown are from one representative experiment out of two performed. $n=5-6$ mice per group. Connecting lines, mean; dots, individual mice. 
Pediatric patients with B-ALL that were treated with CD19 CAR T-cell therapy, and subsequently relapsed with either CD19pos or CD19 $19^{\text {neg }}$ disease, had high levels of TdT expression in leukemic blasts both before and after CAR T-cell therapy (Fig. 4f and Supplementary Table 3). Before CAR T-cell infusion, all patients were extensively pretreated with several lines of therapy, including allogeneic stem-cell transplantation in two patients. Likewise, we observed high TdT levels in leukemic cells from a patient with T-ALL at diagnosis, at time points with measurable minimal residual disease (MRD) after chemotherapy and following relapse (Fig. 4g and Supplementary Table 4). This further suggested that TdT-targeting TCRs might represent an attractive therapeutic option for these patient groups with refractory and relapsed disease and a dismal prognosis.

We next investigated the efficacy of T3-cell treatment on NSG mice stably engrafted with cells derived from a patient with primary B-ALL leukemia (Fig. 5a). Untreated mice and those treated with control MART-1 (DMF5) ${ }^{20}$ or T3 TCR-transduced T cells showed a high and comparable leukemia burden in BM at baseline (untreated, mean 9.7 $\pm 3.4 \%$; DMF5, mean $15.6 \pm 5.7 \%$; T3, mean $15.4 \pm 7.2 \%)$, representing a stringent condition for testing of therapeutic efficacy (Fig. 5d). In a clinical setting TdT TCRs will most probably be introduced into autologous $\mathrm{T}_{\text {cells }}{ }^{1,2}$. Here, we could not obtain sufficient numbers of T cells from the patient sample and we therefore utilized Tcells matched for HLA-A2 expression but otherwise not HLA-matched with ALL. Infused T cells contained mainly naïve and central memory T cells and, on average, $34 \% \mathrm{CD}^{+}$ T cells (Extended Data Fig. 1c), resembling a clinical product facilitating antitumor reactivity in adoptive T-cell therapy ${ }^{21,22}$. However, a high fraction of naïve T cells also drives alloreactivity ${ }^{23,24}$, as does the presence of a high burden of leukemic cells partially HLA mismatched with Tcells. We therefore monitored mice for the likely development of a graft-versus-leukemic effect by comparing leukemic engraftment in the blood of DMF5 (control TCR)-treated relative to untreated mice (Fig. 5b). Detailed end-stage analysis was performed 11 days after T-cell treatment, before the results would be confounded by alloreactivity. At this experimental endpoint, leukemia burden in control mouse groups was very high and comparable in the BM (untreated, mean $42.9 \pm 3.4 \%$; DMF5, mean $40.1 \pm 4.5 \%$ ) whereas it was reduced to as little as $0.009 \pm 0.005 \%$ following treatment with T3 cells (Fig. $5 \mathrm{c}-\mathrm{e}$ ). Leukemia burden in $\mathrm{PB}$ and spleen in untreated and DMF5-cell-treated mice was lower than in BM. Nevertheless, a similar and almost complete elimination of leukemic cells was observed in mice treated with T3 cells (PB, mean $0.003 \pm 0.001 \%$; spleen, mean $0.002 \pm 0.001 \%$ ) (Fig. $5 \mathrm{~d}$ ). Taken together, leukemic burden was reduced to a level considered negative for MRD $(<0.1 \%)$ according to criteria set by the Nordic Society of Paediatric Haematology and Oncology (NOPHO) 2008 protocol (no. NCT00816049). TCR-transduced T cells were detected in the BM, PB and spleen of all treated mice (Fig. 5f), with similar numbers of total mononuclear cells (MNCs) between groups (Fig. 5g). Mouse hematopoiesis, which is suppressed in the BM of individuals with leukemic engraftment, was significantly higher in T3 than in DMF5-treated mice (Fig. 5h).

Normal peripheral B- and T-cell repertoires are TdT negative and were not affected by $\mathrm{T} 3$ or T1 TCRs in in vitro killing assays or during expansion of transduced cells (Fig. $4 \mathrm{~b}$ and Extended Data Fig. 1b). Studies in which the homeostasis of naïve T cells in normal individuals was measured using retrospective ${ }^{14} \mathrm{C}$ dating ${ }^{25}$ support the view that the naïve T-cell repertoire would be sufficiently diverse to sustain peripheral adaptive T-cell immunity for life also in young individuals, and that potential toxic effects on thymocytes would therefore not be a major concern even during prolonged therapy. Thymic contribution to the peripheral repertoire is, however, higher in individuals $<20$ years of age ${ }^{26}$, raising the possibility that toxicity could be higher in this age group. To address this question, we studied the expression of TdT and HLA-A2 during human thymocyte differentiation. Interestingly, we found that HLA-A2 expression was high only on TdT ${ }^{\text {heg }}$ thymocytes, including early double-negative (DN) and single-positive (SP) cells, whereas HLA-A2 expression was downregulated concomitantly with upregulation of TdT on late DN cells transitioning to double-positive (DP) cells (Fig. 6a,b and Extended Data Fig. 9a). We next explored the effect of T3 cell therapy on thymocytes in humanized CD $34^{+}$NSG mice injected with T3- or 1G4-transduced autologous human T cells harvested from mouse spleen (Fig. 6c). $\mathrm{T} 3$ cells were demonstrated to effectively kill a $\mathrm{TdT}^{\text {pos }}$ cell line in vitro before injection (Extended Data Fig. 9b,c). At the end of the experiment, the fraction of $\mathrm{TdT}^{\mathrm{p} o s}$ thymocytes was similar in T3- and 1G4-treated mice. HLA-A2 levels were similarly low on $\mathrm{TdT}^{\text {pos }}$ thymocytes compared to surface $\mathrm{CD}^{+} \mathrm{TdT}^{\text {neg }}$ thymocytes in both experimental groups (Fig. 6d,e and Extended Data Fig. 9d). Correspondingly, only a minor fraction of human thymocytes, replenished by cord blood progenitors in the mouse thymus, were $\mathrm{TdT}^{\text {high }}$ and HLA-A2 $2^{\text {high }}$ in both groups, as was also observed for thymocytes derived from human thymus (Fig. 6a and Extended Data Fig. 9e). The experiment was ended on day 17 after T-cell injections when TCR-transduced T cells were still detectable in PB, with the fraction of 1G4-transduced T cells declining faster than T3-transduced T cells in PB, possibly suggesting low-level antigen stimulation of T3 T cells (Fig. $6 \mathrm{f}$ and Extended Data Fig. 10a,b). No difference between 1G4 and T3 groups was observed following analysis of the distribution of human myeloid and lymphoid lineages in PB, BM, spleen and thymus, as well as cellularity in BM (Extended Data Fig. 10a-g). Further supporting lack of toxicity on hematopoietic stem cells and myeloid progenitor cells, the formation of myeloid and erythroid colonies from adult CD $34^{+} \mathrm{BM}$ progenitor cells was not negatively affected following coculture with T1 or T3 cells (Fig. $6 \mathrm{~g}$ ), unless coculture was performed in the presence of peptide-1 or -3 (Extended Data Fig. 10h).

Fig. 4 | T1 and T3 cells deplete patient-derived cancer cells while sparing normal B and T lymphocytes and nonlineage-committed hematopoietic progenitor cells. a, Representative $t$-SNE plots showing live HLA-A2 ${ }^{\text {pos }}, \mathrm{TdT}^{\text {pos }} \mathrm{B}-\mathrm{ALL}$ tumor cells $\left(\mathrm{CD} 19^{+} \mathrm{CD} 10^{+}\right.$events, left) and T-ALL tumor cells $\left(\mathrm{CD} 5^{+} \mathrm{CD} 7^{+} \mathrm{CD} 99^{+}\right.$and surface $\mathrm{CD} 3^{-} \mathrm{CD} 4^{-}$events, right), normal B cells $\left(\mathrm{CD} 19^{+} \mathrm{CD} 10^{-}\right)$, normal $\mathrm{T}$ cells $\left(\mathrm{CD} 3^{+}\right.$and $\mathrm{CD} 8^{+}$or $\left.\mathrm{CD}^{+}\right)$and $\mathrm{CD} 34^{+}$lin ${ }^{-}$ progenitor cells following $72 \mathrm{~h}$ of coculture with mock-, T1- or T3-transduced T cells (E/T ratio, 1/1), as quantified by flow cytometry. b, Diagnostic samples from 12 patients (Pt.) with HLA-A2 ${ }^{\text {pos }, ~ T d T p o s ~ B-A L L ~ o r ~ T-A L L, ~ a s s a y e d ~ a s ~ d e s c r i b e d ~ i n ~} \mathbf{a}$. Each dot represents the number of live tumor, normal B or T cells after coculture with $\mathrm{T} 1$ (green) or T3 (purple) cells, as percentage of corresponding numbers in cultures treated with mock-transduced T cells (gray). c, Dots showing numbers of live CD34+lin- cells after coculture with $\mathrm{T} 1$ (green) and T3 (purple) cells as percentage of corresponding numbers in cocultures treated with mock-transduced T cells (gray). b,c, Data points represent three or four technical replicates and horizontal lines denote mean. Data shown are from one experiment representative of at least two performed for each patient sample. $\mathbf{d}, t$-SNE plot of PB diagnostic sample from B-ALL patient no. $1 \mathrm{~N}$ after $72 \mathrm{~h}$ of coculture with autologous $\mathrm{T}$ cells transduced with $\mathrm{T} 1, \mathrm{~T} 3$ or mock. $\mathbf{a}, \mathbf{d}$, Inset numbers denote absolute event counts of the indicated cell populations. e, Tandem mass spectrometry fragmentation spectra of TdT peptide-1 and -3 identified from leukemia cells of patient no. 119N. f, Expression of CD19 and TdT in leukemia cells before and after relapse from CD19-specific CAR T-cell therapy in two patients with B-ALL. Ctrl, control. g, Expression of TdT in leukemia cells from a patient with T-ALL at the time of primary diagnosis (Dx) and at relapse after chemotherapy. 


\section{Discussion}

Our study shows that Tcells transduced with TCRs recognizing peptides from the cancer target TdT in the context of allogeneic HLA-A2 efficiently killed TdT ${ }^{\text {pos }} \mathrm{HLA}-\mathrm{A} 2^{\text {pos }}$ patient-derived lymphoblastic leukemia cells of B- and T-cell origin in vitro. While the killing efficacy mediated by TdT TCRs will depend on expression levels of TdT, it will also be affected by a number of other factors, such as the expression level of the restricting HLA-A ${ }^{\star} 02: 01$ a

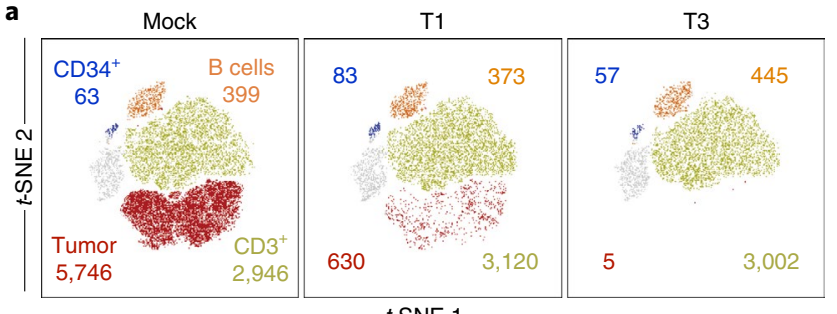

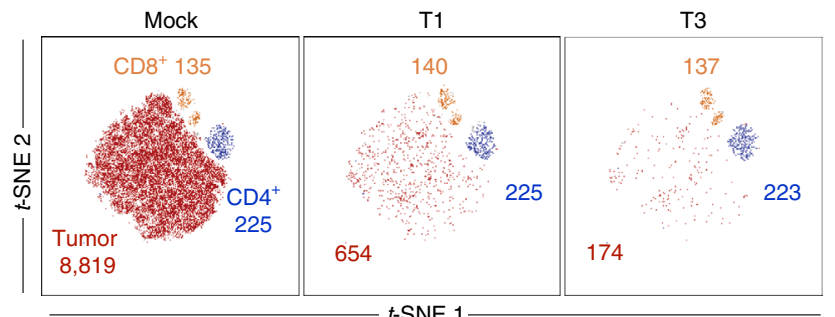

$t$-SNE 1

b

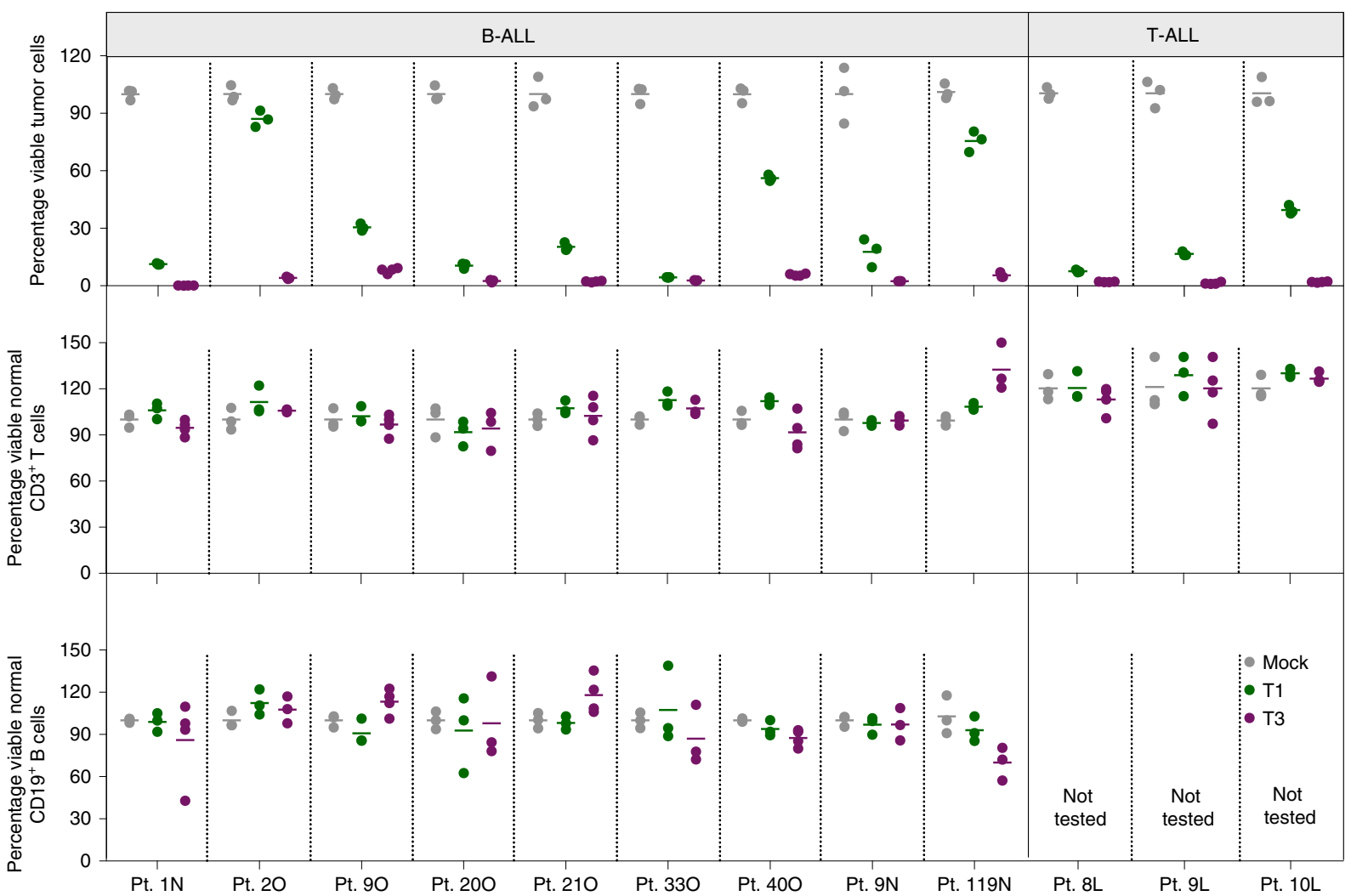

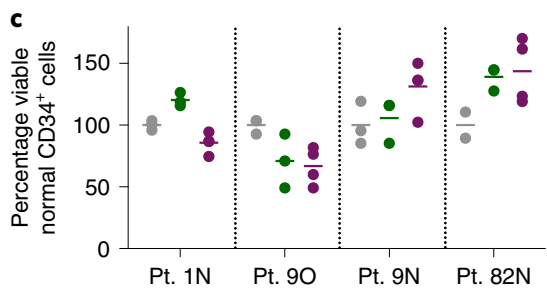

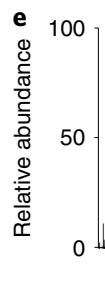
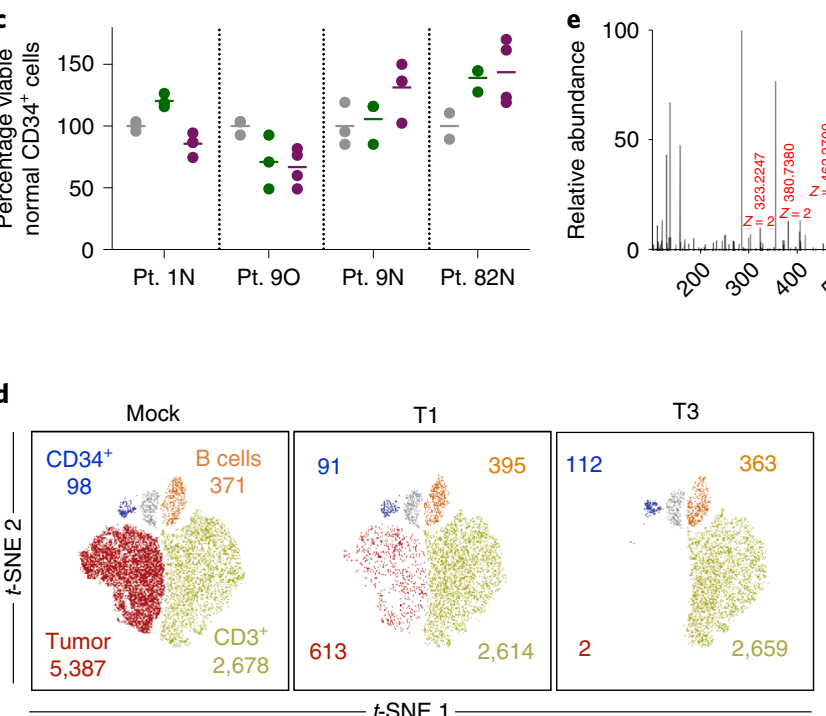

ALYDKTKR (peptide-1)

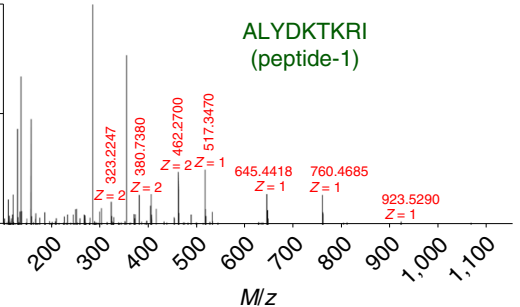

$M / z$

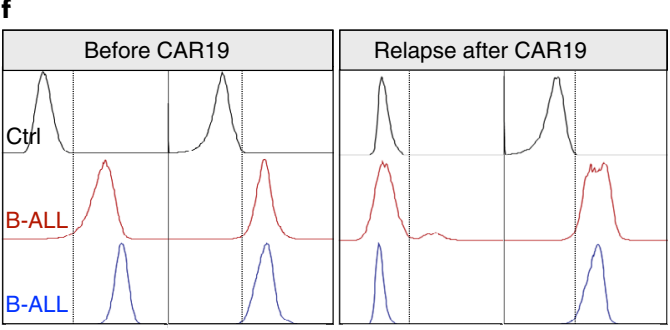

CD19
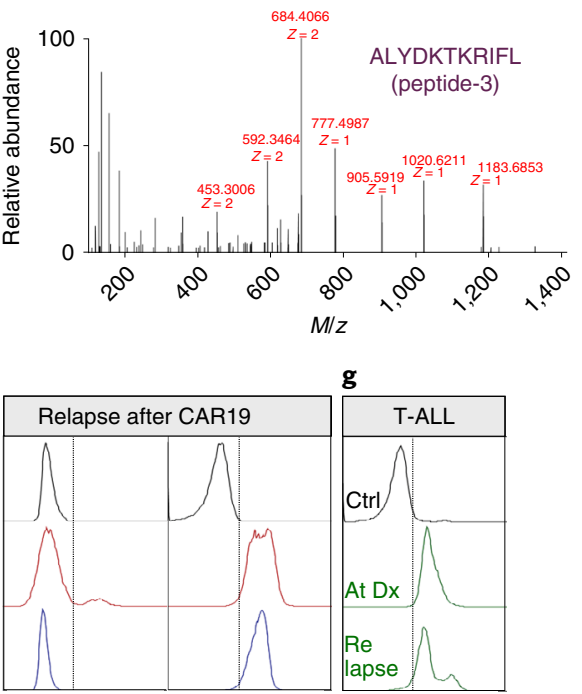

$-\mathrm{TdT}$ 


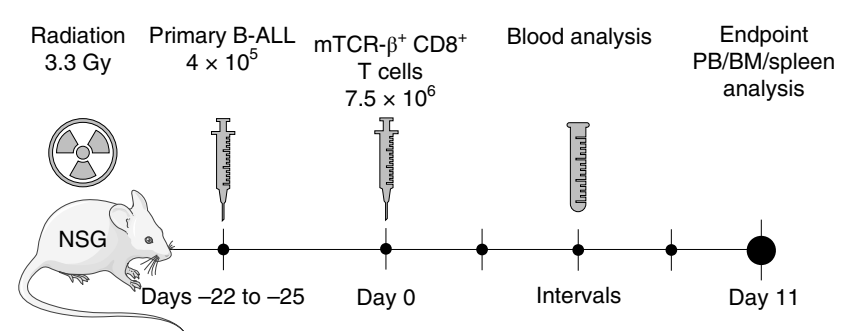

b

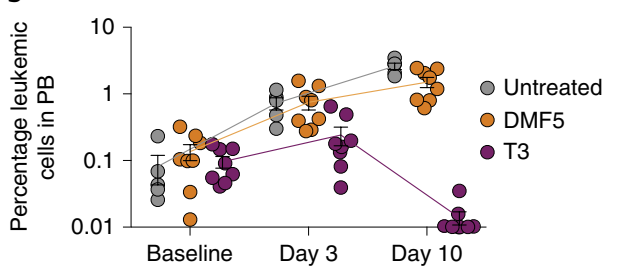

c

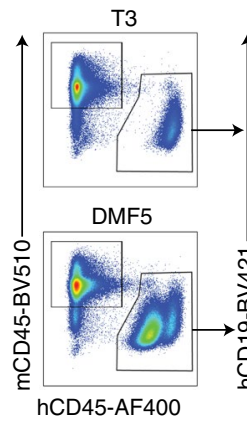

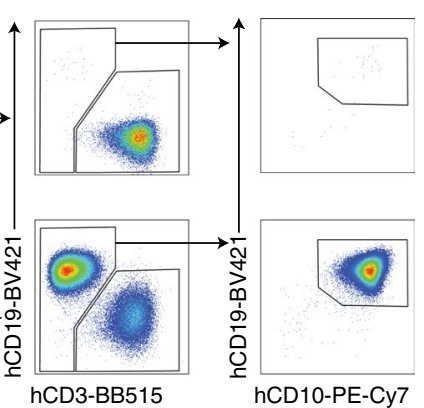

f

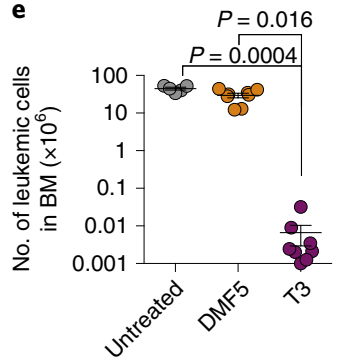

d

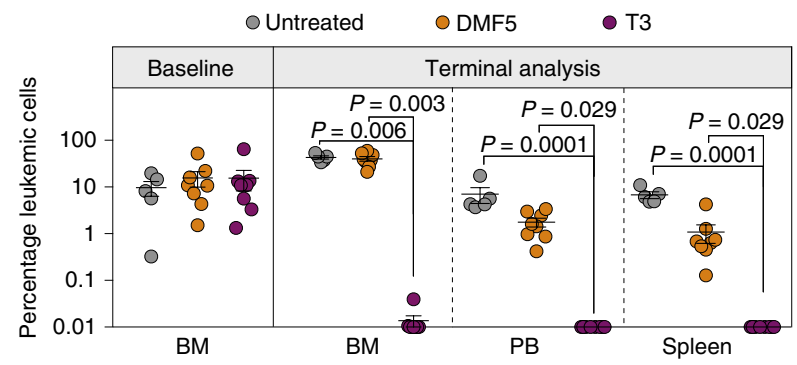

h

Fig. 5 | T3 cells efficiently eliminate primary B-ALL cells in vivo. a, Experimental overview of the PDX model. b, Percentage leukemic cells adjusted for human T cells in PB at baseline and at indicated time points after T-cell infusion. c, Representative FACS plots of viable single MNCs from BM of T3-treated (top) and DMF5-treated (bottom) NSG mice engrafted with primary human B-ALL cells. d, Percentage leukemic cells (hCD45+CD19+CD10 $)$ adjusted for human T cells at baseline (BM) and at terminal analysis on day 11 after T-cell infusion (BM, PB and spleen). e, Number of leukemic cells present in BM. f, Number of TCR-transduced human CD8 $8^{+}$cells in BM, PB and spleen. $\mathbf{g}$, Total numbers of human- and mouse-derived MNCs in BM, PB and spleen. $\mathbf{h}$, Number of mouse CD45+ cells in BM. $\mathbf{b}, \mathbf{d}-\mathbf{h}$, Data are pooled from two independent experiments and presented as mean \pm s.e.m. of untreated ( $n=5$ ), DMF5-treated $(n=8)$ and T3-treated $(n=8)$ mice. Populations identified by flow cytometry according to gating strategy shown in $\mathbf{c}$. Kruskal-Wallis one-way ANOVA by Dunn's multiple comparisons test $(\mathbf{d}, \mathbf{e})$ and two-tailed Mann-Whitney test $(\mathbf{f}-\mathbf{h})$ were performed for statistical analyses.

molecule, the rate of processing of TdT and presentation on HLA as well as the susceptibility of the target cells to T-cell-mediated killing. In light of that, it was reassuring that TdT TCR-expressing T cells were highly efficient at killing a large number of $\mathrm{TdT}^{\text {pos }} \mathrm{ALL}$ cell lines as well as primary ALL cells.

The TdT TCRs also mediated depletion of engrafted leukemia in vivo in three different mouse models of B-ALL. This included a model of primary leukemia with a high pretreatment leukemic burden. We used TCR-transduced T cells containing a high proportion of naïve T cells, which facilitates persistence and antitumor reactivity in adoptive T-cell therapy ${ }^{21,22}$. In a clinical setting TdT TCRs will most probably be introduced into autologous $\mathrm{T}$ cells, but patient-derived T cells were not available in sufficient numbers for the primary diagnosis (PDX) in vivo experiment pursued here. Allogeneic T cells (not HLA-matched except for HLA-A2) containing a high fraction of naïve Tcells will, however, also promote alloreactivity ${ }^{23,24}$. To assess the in vivo efficacy and specificity of TdT TCRs, mice were therefore euthanized and all relevant tissues analyzed at a time point before alloreactivity would considerably confound the antileukemic effects specifically induced by TdT TCR. However, despite a very high leukemic burden in control TCR mice, leukemic cells were almost completely eliminated in TdT TCR-treated mice at this time.
In the two xenograft cell line models we observed no evidence of an alloresponse after 14-21 days (determined by imaging analysis, which may be less sensitive than the flow cytometric assessment of PB used in the PDX model), at which time almost complete elimination of leukemic cells was observed in mice treated with TdT TCRs. These mice were also examined after 2 months, at which time ALL cells remained undetectable in almost all T3-treated mice, providing support for the longer-term efficacy of TdT TCRs. Since untreated and control TCR-treated mice died or had to be euthanized after 2-3 weeks due to leukemia, as expected in aggressive xenograft cell line models, it cannot be ruled out that alloreactivity in part contributes to the long-term effects in such models. In the future, longer follow-up could be studied in PDX models in which TCR-transduced Tcells are derived from the same patient. For T-ALL, in vivo activity of TdT TCRs remains to be tested.

Our data indicate that TCRs could be used in therapy without detrimental toxicity. First, normal Tcells, Bcells and nonlineage-committed $\mathrm{CD} 34^{+}$cells were selectively spared in samples from patients with B- and T-ALL. Second, treatment of humanized mice engrafted with $\mathrm{CD}_{3} 4^{+}$cord blood cells with autologous human TdT TCR-transduced T cells did not reveal a negative impact on normal hematopoiesis-most importantly, myelopoiesis 

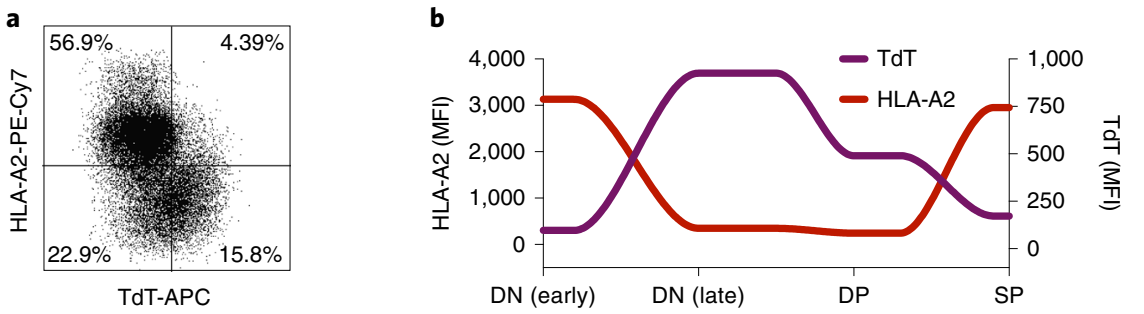

c
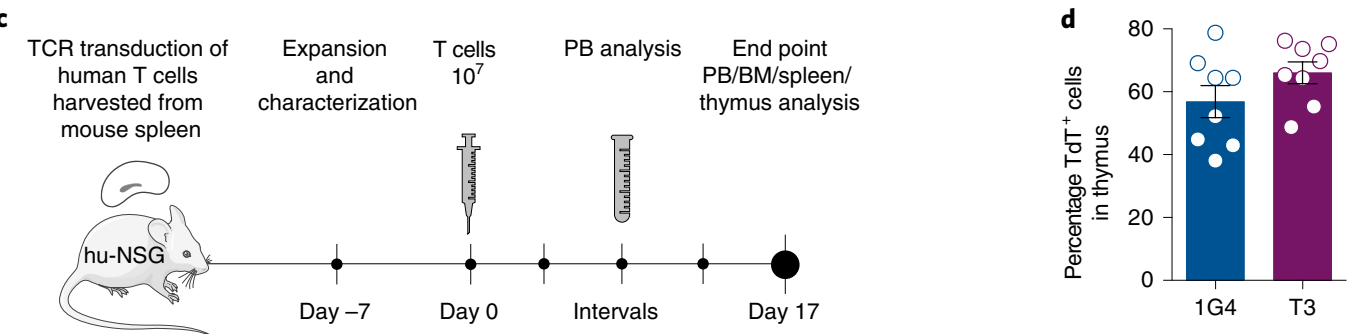

e

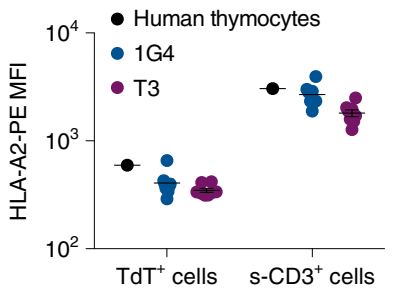

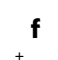

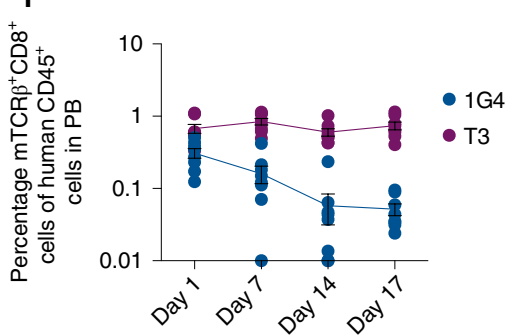

$\mathbf{g}$

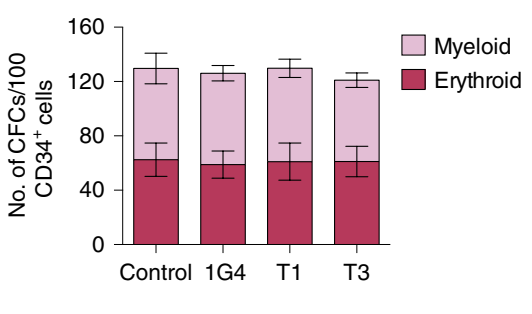

Fig. 6 | T3 cells preserve healthy hematopoiesis in vivo. a,b, FACS analysis of viable human CD19-HLA-DR neg thymocytes (a), and spaghetti-plot illustrating HLA-A2 and TdT mean fluorescence intensity (MFI) at distinct stages of T-cell development (b), defined as shown in Extended Data Fig. 9a, in human thymus. c, Experimental overview of humanized NSG (hu-NSG) mouse model used to investigate the effect of T3-cell therapy on healthy human hematopoiesis. d, Percentage of human TdTpos cells in thymus of hu-NSG mice ( $n=8$ per group). e, HLA-A2 mean fluorescence intensity of TdTos cells (hCD45 $\left.{ }^{+} \mathrm{TdT}^{\mathrm{pos}}\right)$ and surface $\mathrm{CD}^{+}\left(\mathrm{s}-\mathrm{CD} 3^{+}\right)$human thymocytes derived from one normal human thymus and from thymus of $1 \mathrm{G} 4(\mathrm{n}=8)$ and $\mathrm{T} 3$ $(n=8)$-treated humanized mice. $\mathbf{f}$, Percentage of TCR-transduced CD8 ${ }^{+}$cells in PB at indicated days after T-cell infusion ( $n=8$ per group). $\mathbf{g}$, Myeloid and erythroid colonies generated from sorted normal adult human BM CD34 ${ }^{+}$cells ( $n=4$ biological replicates, data pooled from two independent experiments) following coculture with or without $1 \mathrm{G} 4, \mathrm{~T} 1$ or $\mathrm{T} 3$ cells for $72 \mathrm{~h}$ at an $\mathrm{E} / \mathrm{T}$ ratio of $2 / 1$. All data are presented as mean $\pm \mathrm{s}$.e.m.; dots in $\mathbf{d}-\mathbf{f}$ represent individual mice at terminal analysis on day 17 post treatment, unless otherwise stated.

or T-cell development. These data are supported by in vitro results showing (1) lack of toxicity on myelopoiesis in colony-forming assays and (2) that the majority of thymocytes are $\mathrm{TdT}^{\text {neg }}$ and that HLA-A2 is downregulated concomitantly with upregulation of TdT, with only a minority of thymocytes expressing both at high levels.

Specificity for TdT peptides and HLA-A2-restriction was confirmed by loss of reactivity to $\mathrm{TdT}^{\text {pos }}$ leukemia cells in which $\mathrm{TdT}$ was knocked out or HLA-A2 was absent, and cognate peptides presented on HLA of primary leukemia cells were directly identified by MS. A variety of HLA-A2 $2^{\text {pos }}$ cell lines that were $\mathrm{TdT}^{\text {neg }}$ were not recognized, and mapping of TCR reactivity did not identify any naturally occurring 9- or 11-mer peptide in the human proteome that matched the combinations of substituted peptides to which T1 or T3 were reactive. Taken together, the data indicating high efficacy and specificity of TdT TCRs, combined with the unique expression profile of $\mathrm{TdT}$, open new possibilities for the treatment of $\mathrm{TdT}^{\mathrm{pos}}$ acute leukemia, including patient groups that currently have a dismal prognosis. While our in vitro and in vivo analyses provided no evidence for a major negative impact of TdT TCRs on thymopoesis, the first clinical testing may preferentially focus on adolescent or adult patients in whom thymic output is expected to be modest or very low ${ }^{25}$.

In spite of the success of CAR T-cell therapies in B-cell malignancies, there are currently few options for the $40-50 \%$ of patients that relapse $e^{2,5}$, and B-ALL patients $>25$ years are currently ineligible for commercially available CAR $19 \mathrm{~T}$ cell therapy due to toxicity concerns. Patients with T-ALL that either have refractory disease, a relapse after allogeneic stem-cell transplantation or very early after first-line therapy, or MRD after relapse induction and consolidation chemotherapy, also have a very poor prognosis. This has prompted the development of CARs targeting pan-T-cell markers such as CD7, CD5, CD3 and CD4 (ref. ${ }^{27}$ ). Although fratricide-resistant CAR T cells lacking targets can be generated by genetic engineering $^{28,29}$, the toxicities related to prolonged T-cell depletion might prove unacceptable in the clinic. CAR T cells targeting CD1a ${ }^{30}$, not expressed on mature Tcells, might prove attractive for a smaller subset of patients suffering from cortical T-ALL, but that does not include other T-ALL subtypes with higher risk and worse prognosis $^{10,31}$. A CAR targeting the TCR- $\beta$ chain constant-domain 1 , that leaves normal Tcells expressing TRBC2 unaffected, is currently under test in a clinical trial including mature T-cell non-Hodgkin lymphoma (no. NCT03590574). However, this CAR is not suitable for T-ALL because only a minority of T-ALL cases have surface expression of $\mathrm{TCR}^{27}$.

Off-target toxicity is a serious concern regarding therapeutic TCRs. This risk has proven particularly high for affinity-enhanced TCRs, in some cases causing lethal cross-reactivity in heart and brain $^{32,33}$, and might be related to the fact that such TCRs have not undergone negative thymic selection against any HLA molecule. Due to the inherent degeneracy of TCRs, monospecificity 
is probably impossible ${ }^{34}$. Nevertheless, recent clinical studies have provided proof of concept for safe and efficacious TCRs ${ }^{19,35,36}$. The goal is thus to identify TCRs that do not show clinically important cross-reactivity. In contrast to affinity-enhanced TCRs, allorestricted TCRs have been negatively selected on up to 12 different HLA alleles. This depletion is substantial, as sequence comparison between, for example, HLA-A alleles shows that TCR contact residues are highly conserved ${ }^{37}$. The highly selective killing mediated by the TdT-specific TCRs T1 and T3 refutes the idea that allorecognition is inherently more cross-reactive than conventional TCR recognition, and the data are supported by structural analysis of allogeneic TCR-pMHC complexes ${ }^{38,39}$. Pioneering studies by Sadovnikova and Stauss $^{40}$, and later by us $^{41,42}$ and others ${ }^{43}$, suggest that allorestricted TCRs can be utilized in cancer immunotherapy. However, the dogma that allo-HLA-restricted TCRs are inherently cross-reactive and mainly recognize the HLA molecule itself has prevailed, limiting therapeutic utility. The question has thus remained whether the few identified allo-HLA-restricted TCRs that seemed to be peptide specific represent rare exceptions, or whether peptide specificity has not been sufficiently investigated. Recent advances in technologies for mapping of TCR reactivity allow for highly sensitive identification of peptides to which a candidate therapeutic TCR might be cross-reactive. Screening of TCR reactivity to unbiased peptide libraries, including yeast two-hybrid assays ${ }^{44}$ and genome-wide libraries introduced into target cells by lentivirus ${ }^{45}$, demonstrated that the tested TCRs cross-reacted to only those peptides harboring an amino acid sequence partly shared with the cognate peptide. Moreover, studies investigating binding of peptide-MHC tetramers with single versus multiple simultaneous amino acid substitutions showed that bioinformatic searches for combinations of hits for single amino acid substitutions, such as that performed for TdT TCRs, were sufficient to identify candidate cross-reactive peptides ${ }^{46}$.

Cell-type-specific antigens are often, unlike cancer testis antigens $^{47}$, homogeneously expressed in malignant cells. Targeting of broadly expressed lineage markers may, however, be prohibitively toxic. The identification of TCRs that efficiently and selectively recognize $\mathrm{TdT}$ paves the way for targeting of cell-type-specific, intracellular antigens that are transiently expressed during differentiation. TdT-specific TCRs could represent an attractive therapeutic option for patients with B-ALL ineligible for, or relapsing from, CD19-specific CAR T-cell therapy, or for patients with T-ALL relapsing from chemotherapy or allogeneic stem-cell transplantation and for whom no cellular immunotherapy currently exists. If successful, the patient groups that might benefit from such T-cell therapy could be expanded.

\section{Online content}

Any methods, additional references, Nature Research reporting summaries, source data, extended data, supplementary information, acknowledgements, peer review information; details of author contributions and competing interests; and statements of data and code availability are available at https://doi.org/10.1038/ s41587-021-01089-x.

Received: 25 March 2021; Accepted: 2 September 2021; Published online: 6 December 2021

\section{References}

1. Maude, S. L. et al. Tisagenlecleucel in children and young adults with B-cell lymphoblastic leukemia. N. Engl. J. Med. 378, 439-448 (2018).

2. Grupp, S. A. et al. Updated analysis of the efficacy and safety of tisagenlecleucel in pediatric and young adult patients with relapsed/refractory ( $\mathrm{r} / \mathrm{r}$ ) acute lymphoblastic leukemia. Blood 132, 895-895 (2018).

3. Orlando, E. J. et al. Genetic mechanisms of target antigen loss in CAR19 therapy of acute lymphoblastic leukemia. Nat. Med. 24, 1504-1506 (2018)

4. Shah, N. N. \& Fry, T. J. Mechanisms of resistance to CAR T cell therapy. Nat. Rev. Clin. Oncol. 16, 372-385 (2019).
5. Ghorashian, S. et al. Enhanced CAR T cell expansion and prolonged persistence in pediatric patients with ALL treated with a low-affinity CD19 CAR. Nat. Med. 25, 1408-1414 (2019).

6. Fry, T. J. et al. CD22-targeted CAR T cells induce remission in B-ALL that is naive or resistant to CD19-targeted CAR immunotherapy. Nat. Med. 24, 20-28 (2018).

7. Quist-Paulsen, P. et al. T-cell acute lymphoblastic leukemia in patients 1-45 years treated with the pediatric NOPHO ALL2008 protocol. Leukemia 34, 347-357 (2020)

8. Sellar, R. S. et al. Relapse in teenage and young adult patients treated on a paediatric minimal residual disease stratified ALL treatment protocol is associated with a poor outcome: results from UKALL2003. Br. J. Haematol. 181, 515-522 (2018).

9. Komori, T., Okada, A., Stewart, V. \& Alt, F. W. Lack of N regions in antigen receptor variable region genes of TdT-deficient lymphocytes. Science 261, 1171-1175 (1993).

10. Coustan-Smith, E. et al. Early T-cell precursor leukaemia: a subtype of very high-risk acute lymphoblastic leukaemia. Lancet Oncol. 10, 147-156 (2009)

11. Drexler, H. G., Menon, M. \& Minowada, J. Incidence of TdT positivity in cases of leukemia and lymphoma. Acta Haematol. 75, 12-17 (1986).

12. Patel, J. L. et al. The immunophenotype of T-lymphoblastic lymphoma in children and adolescents: a Children's Oncology Group report. $\mathrm{Br}$. J. Haematol. 159, 454-461 (2012).

13. Bene, M. C. et al. Immunophenotyping of acute leukemia and lymphoproliferative disorders: a consensus proposal of the European LeukaemiaNet Work Package 10. Leukemia 25, 567-574 (2011).

14. Pellin, D. et al. A comprehensive single cell transcriptional landscape of human hematopoietic progenitors. Nat. Commun. 10, 2395 (2019).

15. Monaco, G. et al. RNA-seq signatures normalized by mRNA abundance allow absolute deconvolution of human immune cell types. Cell Rep. 26, 1627-1640 (2019).

16. Ali, M. et al. Induction of neoantigen-reactive T cells from healthy donors. Nat. Protoc. 14, 1926-1943 (2019).

17. Cohen, C. J., Zhao, Y., Zheng, Z., Rosenberg, S. A. \& Morgan, R. A. Enhanced antitumor activity of murine-human hybrid T-cell receptor (TCR) in human lymphocytes is associated with improved pairing and TCR/CD3 stability. Cancer Res. 66, 8878-8886 (2006).

18. Jin, B. Y. et al. Engineered T cells targeting E7 mediate regression of human papillomavirus cancers in a murine model. JCI Insight 3, e99488 (2018).

19. Rapoport, A. P. et al. NY-ESO-1-specific TCR-engineered T cells mediate sustained antigen-specific antitumor effects in myeloma. Nat. Med. 21, 914-921 (2015).

20. Johnson, L. A. et al. Gene transfer of tumor-reactive TCR confers both high avidity and tumor reactivity to nonreactive peripheral blood mononuclear cells and tumor-infiltrating lymphocytes. J. Immunol. 177, 6548-6559 (2006).

21. Sommermeyer, D. et al. Chimeric antigen receptor-modified $\mathrm{T}$ cells derived from defined $\mathrm{CD} 8^{+}$and $\mathrm{CD} 4^{+}$subsets confer superior antitumor reactivity in vivo. Leukemia 30, 492-500 (2016).

22. Busch, D. H., Fräßle, S. P., Sommermeyer, D., Buchholz, V. R. \& Riddell, S. R. Role of memory $\mathrm{T}$ cell subsets for adoptive immunotherapy. Semin. Immunol. 28, 28-34 (2016).

23. Bleakley, M. et al. Outcomes of acute leukemia patients transplanted with naive T cell-depleted stem cell grafts. J. Clin. Invest. 125, 2677-2689 (2015).

24. Foster, A. E. et al. Human CD62 $\mathrm{L}^{-}$memory T cells are less responsive to alloantigen stimulation than $\mathrm{CD} 62 \mathrm{~L}^{+}$naive $\mathrm{T}$ cells: potential for adoptive immunotherapy and allodepletion. Blood 104, 2403-2409 (2004).

25. Mold, J. E. et al. Cell generation dynamics underlying naive T-cell homeostasis in adult humans. PLoS Biol. 17, e3000383 (2019).

26. Bains, I., Antia, R., Callard, R. \& Yates, A. J. Quantifying the development of the peripheral naive $\mathrm{CD} 4^{+} \mathrm{T}$-cell pool in humans. Blood 113, 5480-5487 (2009).

27. Scherer, L. D., Brenner, M. K. \& Mamonkin, M. Chimeric antigen receptors for T-cell malignancies. Front. Oncol. 9, 126 (2019).

28. Cooper, M. L. et al. An 'off-the-shelf' fratricide-resistant CAR-T for the treatment of $\mathrm{T}$ cell hematologic malignancies. Leukemia 32, 1970-1983 (2018).

29. Gomes-Silva, D. et al. CD7-edited T cells expressing a CD7-specific CAR for the therapy of T-cell malignancies. Blood 130, 285-296 (2017).

30. Sanchez-Martinez, D. et al. Fratricide-resistant CDla-specific CAR T cells for the treatment of cortical T-cell acute lymphoblastic leukemia. Blood 133, 2291-2304 (2019).

31. Marks, D. I. \& Rowntree, C. Management of adults with T-cell lymphoblastic leukemia. Blood 129, 1134-1142 (2017).

32. Cameron, B. J. et al. Identification of a Titin-derived HLA-A1-presented peptide as a cross-reactive target for engineered MAGE A3-directed T cells. Sci. Transl. Med. 5, 197ra103 (2013).

33. Morgan, R. A. et al. Cancer regression and neurological toxicity following anti-MAGE-A3 TCR gene therapy. J. Immunother. 36, 133-151 (2013). 
34. Wooldridge, L. et al. A single autoimmune T cell receptor recognizes more than a million different peptides. J. Biol. Chem. 287, 1168-1177 (2012).

35. Robbins, P. F. et al. Tumor regression in patients with metastatic synovial cell sarcoma and melanoma using genetically engineered lymphocytes reactive with NY-ESO-1. J. Clin. Oncol. 29, 917-924 (2011).

36. Chapuis, A. G. et al. T cell receptor gene therapy targeting WT1 prevents acute myeloid leukemia relapse post-transplant. Nat. Med. 25, 1064-1072 (2019).

37. Stauss, H. J. Immunotherapy with CTLs restricted by nonself MHC. Immunol. Today 20, 180-183 (1999).

38. Wang, Y. et al. How an alloreactive T-cell receptor achieves peptide and MHC specificity. Proc. Natl Acad. Sci. USA 114, E4792-E4801 (2017).

39. Simpson, A. A. et al. Structural and energetic evidence for highly peptide-specific tumor antigen targeting via allo-MHC restriction. Proc. Natl Acad. Sci. USA 108, 21176-21181 (2011).

40. Sadovnikova, E. \& Stauss, H. J. Peptide-specific cytotoxic T lymphocytes restricted by nonself major histocompatibility complex class I molecules: reagents for tumor immunotherapy. Proc. Natl Acad. Sci. USA 93, 13114-13118 (1996).

41. Abrahamsen, I. W. et al. Targeting B cell leukemia with highly specific allogeneic T cells with a public recognition motif. Leukemia 24, 1901-1909 (2010).

42. Kumari, S. et al. Alloreactive cytotoxic T cells provide means to decipher the immunopeptidome and reveal a plethora of tumor-associated self-epitopes. Proc. Natl Acad. Sci. USA 111, 403-408 (2014).

43. Amir, A. L. et al. PRAME-specific allo-HLA-restricted T cells with potent antitumor reactivity useful for therapeutic T-cell receptor gene transfer. Clin. Cancer Res. 17, 5615-5625 (2011).
44. Birnbaum, M. E. et al. Deconstructing the peptide-MHC specificity of T cell recognition. Cell 157, 1073-1087 (2014).

45. Kula, T. et al. T-Scan: a genome-wide method for the systematic discovery of T cell isotopes. Cell 178, 1016-1028 (2019).

46. Bentzen, A. K. et al. T cell receptor fingerprinting enables in-depth characterization of the interactions governing recognition of peptide-MHC complexes. Nat. Biotechnol. 36, 1191-1196 (2018).

47. Sigalotti, L. et al. Intratumor heterogeneity of cancer/testis antigens expression in human cutaneous melanoma is methylation-regulated and functionally reverted by 5-aza-2'-deoxycytidine. Cancer Res. 64, 9167-9171 (2004).

Publisher's note Springer Nature remains neutral with regard to jurisdictional claims in published maps and institutional affiliations.

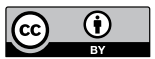

Open Access This article is licensed under a Creative Commons Attribution 4.0 International License, which permits use, sharing, adaptation, distribution and reproduction in any medium or format, as long as you give appropriate credit to the original author(s) and the source, provide a link to the Creative Commons license, and indicate if changes were made. The images or other third party material in this article are included in the article's Creative Commons license, unless indicated otherwise in a credit line to the material. If material is not included in the article's Creative Commons license and your intended use is not permitted by statutory regulation or exceeds the permitted use, you will need to obtain permission directly from the copyright holder. To view a copy of this license, visit http://creativecommons. org/licenses/by/4.0/.

(C) The Author(s) 202 


\section{Methods}

This study was approved by the Regional Committee for Medical and Health Research Ethics (REC) South-East, Norway (nos. 2018/879, 2018/1246, 2019/31516), the Institutional Review Board and the Data Protection Officer, Oslo University Hospital, Swedish Ethical Review Authority, Stockholm (no. EPN 2018/901-31) and was performed in accordance with the Declaration of Helsinki.

Primary patient cells, healthy blood donor cells and cell lines. Pediatric and young adult relapsed/refractory patients with B-ALL were enrolled into and treated according to CAR T-cell trials ClinicalTrials.gov Identifier nos. NCT02435849 and NCT03123939, and pediatric patients with T-ALL according to NOPHO-ALL-2008 (no. NCT00816049). Institutional review board and REC approvals for the use of primary human diagnostic blood and BM samples from pediatric and adult patients in the study were obtained, as was informed written consent from patients or their guardians. Thymocytes were isolated from human thymus removed as a consequence of routine procedures for open cardiac surgery to correct a congenital cardiac defects (in an otherwise healthy child) following informed written consent from guardians (ethical approval no. 2019/31516). PB MNCs (PBMCs) from healthy donor buffy coats were obtained from the blood bank of Oslo University Hospital, and PB or BM MNCs from leukemia patients were obtained from biobanked, cryopreserved material (ethical approval nos. 2018/879 and 2018/1246). MNCs were isolated by density-gradient centrifugation (Axis-Shield) and were typed for HLA-A2 expression by flow cytometry. EBV-LCLs were generated from HLA-A2 $2^{\text {pos }}$ and HLA-A2 $2^{\text {neg }} P B M C s$ as described previously ${ }^{48}$. Thymic tissue was cleaned from blood clots, connective tissue, fat tissue and necrotic tissue and cut into small pieces that were gently triturated with a 1-l micropipette attached to a wide-bore pipette tip to release thymocytes into cold RPMI-1640 medium. Thymocytes were washed twice in medium and then cryopreserved.

The following cell lines were used in the study: NALM-6, BV173, EBV-LCL HPB-ALL, T2, REH, RD, U-2 OS, FM-6, HeLa, HaCaT, COLO688, EA.hy926, U-87 MG, Daoy, HCT-116, CHP-212, MCF7, K562, RS4;11 and Phoenix-AMPHO. Cell lines were obtained from the American Tissue Culture Collection and Deutsche Sammlung von Mikroorganismen und Zellkulturen, and cryopreserved aliquots were labeled according to passage. Only low passages (one to four) were used to start cultures. The identity of cell lines with high passages (five or higher) used experimentally was ascertained by short tandem repeat DNA profiling, a service provided by Labcorp DNA Identification Lab (formerly Genetica, https:// cellineauthentication.com/). Cell lines were cultured in humidified cell incubators containing $5 \% \mathrm{CO}_{2}$ at $37^{\circ} \mathrm{C}$ in media as instructed by the supplier, and regularly tested for mycoplasma contamination.

Induction of antigen-specific $\mathrm{T}$ cells. Induction of $\mathrm{T}$ cells reactive to $\mathrm{Td} \mathrm{T}$ peptides and generation of cytotoxic $\mathrm{T}$-cell lines and clones were performed as previously described $^{16}$, with some modifications. Briefly, monocytes were isolated from PBMCs of HLA-A2 ${ }^{\text {pos }}$ healthy donors on day 4 using CD14-reactive microbeads and AutoMACS Pro Separator (Miltenyi Biotec), and cultured for 3 days in CellGro GMP DC medium (CellGenix) supplemented with $1 \%(\mathrm{v} / \mathrm{v})$ human serum (HS, Trina biotech), $1 \%$ (v/v) penicillin/streptomycin, $50 \mathrm{IU} \mathrm{ml}^{-1}$ interleukin (IL)-4 (PeproTech) and $800 \mathrm{IU} \mathrm{ml}^{-1} \mathrm{GM}-\mathrm{CSF}$ (Genzyme). Subsequently MoDCs were matured for $14-16 \mathrm{~h}$ by the addition of lipopolysaccharide (Sigma-Aldrich) and IFN- $\gamma$ (PeproTech) to final concentrations of $10 \mathrm{ng} \mathrm{ml}^{-1}$ and $100 \mathrm{IU} \mathrm{ml}^{-1}$, respectively. On day -1 , naive $\mathrm{CD} 8^{+} \mathrm{T}$ cells were isolated from PBMCs from HLA-A2 ${ }^{\text {neg }}$ donors by the AutoMACS Pro Separator and CD8 ${ }^{+} \mathrm{T}$-cell isolation kit premixed with CD45RO- and CD57-reactive beads (Miltenyi Biotec). On day 0, MoDCs were harvested, electroporated with mRNA encoding full-length TdT and cocultured with naïve T cells in DC-T cell medium supplemented with $30 \mathrm{ng} \mathrm{ml}^{-1}$ IL-21 (PeproTech) at a DC/T-cell ratio of 1/4. Parallel control cocultures were initiated with MoDCs transfected with irrelevant mRNA. Between days 8 and 10, cocultures were screened for the presence of TdT pMHC multimer-reactive CD8 ${ }^{+}$ Tcells. pMHC multimers labeled with phycoerythrin (PE) and allophycocyanin (APC) were prepared in-house as described previously ${ }^{49,50}$. Live $\mathrm{CD} 8^{+} \mathrm{T}$ cells staining positively for PE- and APC-conjugated pMHC multimers were sorted subsequently by fluorescence activated cell sorting (FACS).

Sorting and cloning of pMHC multimer ${ }^{+} \mathrm{CD8}^{+} \mathrm{T}$ cells. Single-cell cloning of peptide- 1 and peptide- 3 MHC multimer ${ }^{+} \mathrm{CD}^{+}$T cells was performed as described previously ${ }^{16}$. Briefly, PBMCs from three different donors were mixed in $1 / 1 / 1$ ratio, irradiated with $35 \mathrm{~Gy}$, washed and resuspended in X-vivo 20 medium (Lonza, BioNordika) supplemented with 5\% (v/v) human serum and $1 \%(\mathrm{v} / \mathrm{v})$ penicillin/streptomycin (T-cell medium). Feeder cells were added to 96 -well tissue-culture-treated plates $\left(0.2 \times 10^{6}\right.$ cells per well in a volume of $100 \mu \mathrm{l}$ ) and $100 \mu \mathrm{l}$ of T-cell medium containing $2 \mu \mathrm{g} \mathrm{m}^{-1}$ phytohaemagglutinin (Remel Thermo Scientific), $80 \mathrm{ng} \mathrm{ml}^{-1} \mathrm{IL}-2$ (R\&D Systems), and $4 \mathrm{ng} \mathrm{ml}^{-1} \mathrm{IL}-15$ (PeproTech) was added to the feeder cells. MoDC/T-cell cocultures were harvested and stained with anti-CD3 and -CD8a antibodies, and PE- and APC-conjugated pMHC multimers. CD8 ${ }^{+}$and double-pMHC multimer ${ }^{+}$cells were sorted as single cells into 96-well plates containing feeder cells using either FACS Aria II (BD Biosciences) or a SH800 cell sorter (Sony Biotechnology). Every 7 days, cultures were supplied with fresh T-cell medium containing IL-2 and IL-15 and expanding clones were identified by microscopy. On day 14 after FACS, growing clones were restimulated with feeder cells prepared as described above. T-cell clones were stained with relevant pMHC multimers. As negative controls, peptide-1 reactive clones were stained with peptide- 3 MHC multimers and vice versa. To assess functionality, T-cell clones were stimulated with EBV-LCLs pulsed with relevant peptides and the NALM- 6 cell line naturally expressing TdT, and assessed for upregulation of CD137.

TCR sequencing and cloning. Paired TCR- $\alpha$ and $-\beta$ chains from three clones reactive to peptide- 1 and one reactive to peptide- 3 were amplified using a protocol described previously, which was modified and adapted for the targeted amplification of TCR $\alpha$ and $\beta$ transcripts ${ }^{51,52}$. In brief, RNA was extracted and processed to obtain TCR-specific complementary DNA. Four pairs of TCR- $\alpha / \beta$ constant-domain-specific primers were used to run PCR with reverse transcription for each clone, followed by the addition of a poly-G tail and template switch to obtain double-stranded DNA. Finally, two rounds of nested PCR amplification were performed using additional constant-domain primers and adapter primers annealing to an anchor sequence introduced in the poly-G domain. A Kappa Illumina kit was utilized to prepare libraries, which were later sequenced on an Illumina MiSeq. MiTCR script was used to analyze sequencing data, and an in-house python script TCRprimer was used to reconstruct full-length TCR chains as described previously $y^{51,53}$. Output was manually verified for each sample in IMGT/V-Quest ${ }^{54}$. Variable TCR- $\alpha$ and $-\beta$ fragments of identified TCRs were codon-optimized, synthesized and cloned by Genscript.

Gene transfer to human PBMCs and cell lines. T1 and T3 TCRs were transduced into HLA-A2 $2^{\text {pos }}$ donor-derived and patient-derived PBMCs. 1G4 and DMF5 TCRs were also transduced into HLA-A2 ${ }^{\text {pos }}$ donor-derived PBMCs as controls for in vivo experiments. For stimulation of human PBMCs, 6- or 12-well tissue-culture-treated plates were coated with anti-CD3 (clone OKT3, eBioscience) and anti-CD28 (clone CD28.6, eBioscience) antibodies. PBMCs $\left(2 \times 10^{6} \mathrm{cells} \mathrm{ml}^{-1}\right)$ in T-cell medium supplemented with IL-7 and IL-15 (5 $\mathrm{ng} \mathrm{ml}^{-1}$ each, PeproTech) were added to antibody-coated plates and incubated at $37^{\circ} \mathrm{C}$ and $5 \% \mathrm{CO}_{2}$ for $72 \mathrm{~h}$. For generation of retroviral supernatants, $4 \times 10^{6}$ Phoenix-AMPHO packaging cells were plated in $10-\mathrm{cm}$ Petri dishes for $24 \mathrm{~h}$, and cells were transfected with $\gamma$-retroviral vector DNA and mixed in X-tremeGENE 9 DNA Transfection reagent (Roche Diagnostics) and Opti-MEM. The following day, medium was refreshed and cells were incubated at $32^{\circ} \mathrm{C}$ and $5 \% \mathrm{CO}_{2}$ for $24 \mathrm{~h}$. Subsequently, PBMCs were harvested, resuspended in T-cell medium supplemented with IL-7 and IL-15, mixed with retroviral supernatant, placed in non-tissue-culture-treated 6-well plates precoated with Retronectin $\left(20 \mathrm{\mu g} \mathrm{ml}^{-1}\right.$, Takara) and spinoculated at $900 \mathrm{~g}$ for $60 \mathrm{~min}$. A second spinoculation was performed the following day with fresh retroviral supernatant, and transduction efficiency was determined after 3-5 days by staining with anti-mouse TCR- $\beta$ chain antibody and/or pMHC multimer followed by flow cytometry. Before functional experiments, cells were cultured for $48-72 \mathrm{~h}$ in T-cell medium containing low concentrations of cytokines $\left(0.5 \mathrm{ng} \mathrm{ml}^{-1}\right.$ IL-7 and IL-15). Alternatively, cells were frozen for later experiments.

Retroviral supernatants containing viral DNA encoding full-length HLA-A2 and TdT were also produced as described above and utilized to transduce REH, RD, HeLa, K562, HaCaT, COLO688, EA.hy926 and HPB-ALL cell lines with HLA-A2, and EBV-LCL with TdT. For in vivo experiments, the BV173 and NALM-6 cell lines were stably transduced to express firefly luciferase and GFP, and were designated as BV173 $3^{\text {ffuc-eGFP }}$ and NALM-6 $6^{\text {ffuc-eGFP. }}$. All transduced cell lines were subsequently purified by FACS sorting, expanded and frozen for use in later experiments.

Complementary DNA for TdT and HLA-A2 was cloned into the pCIpA102 vector for mRNA production, as previously described ${ }^{42}$

Antibodies and flow cytometry. Flow cytometry was performed on either a BD LSR II flow cytometer (BD Biosciences), FACSCanto II (BD Biosciences), FACS Aria Fusion (BD Biosciences) or MACSQuant (Miltenyi Biotec), and data were analyzed using FlowJo (TreeStar) or FACS DIVA (BD Biosciences) software. For surface antibody staining, antibodies were added to cells for 15-20 min on ice followed by washing steps. For intracellular staining, cells were suspended in Cytofix/Cytoperm (BD Bioscience) solution for $20 \mathrm{~min}$, washed with Perm/ Wash buffer (BD Bioscience) and then stained with antibodies. The following fluorescently conjugated anti-human antibodies were acquired from BD Biosciences or BioLegend, unless otherwise specified: anti-CD14 (no. HCD14, 61D3, eBioscience), -HLA-A2 (no. BB7.2), -CD62L (no. DREG-56), CD56 (no. HCD56, B159), -CD57 (no. HNK-1), -CD45RO (no. UCHL1), -CD45RA (no. HI100), -CCR7 (no. 150503), -CD137 (no. 4B4-1), -CD45 (no. HI30), -TdT (no. E17-1519), -CD10 (no. HI10a), -CD19 (no. HIB19, SJ25C1), -CD38 (no. HIT2), -CD34 (no. 581, 8G12), -CD1a (no. HI149), -CD2 (no. S5.2, RPA-2.10), -CD3 (no. UCHT1, OKT3, HIT3a), -CD8a (no. RPA-T8), -CD4 (no. RPA-T4), -CD5 (no. UCHT2, L17F12), -CD7 (no. M-T701), -CD33 (no. WM-53), -CD11b (no. ICRF44), -CD20 (no. 2H7), -CD235a,b (no. HIR2) anti-mouse CD45 (no. 30-F11), -CD45.1 (no. A20), -CD99 (no. DN16, Bio Rad), Ter-119 (no. TER-119) and -CD3 (no. SK7, eBioscience). Anti-mouse TCR- $\beta$ chain PE (no. H57-597, BD 
Biosciences) was used to test the transduction efficiency of T1 and T3 TCRs in human cells, and to monitor transduced T cells used for in vivo treatment in mice. Live/Dead Fixable Near-IR Dead Cell Stain kit (Life Technologies), Live/Dead Fixable Aqua Dead Cell Stain kit (Life Technologies), DAPI (Invitrogen) or 7-AAD (BioLegend) was used to exclude dead cells in flow-cytometry experiments. A list of antibodies used for flow cytometry or ELISA is provided in Supplementary Table 5, including information about supplier, species, clone name, fluorochromes, dilution factor used and application. Unconjugated anti-HLA class I antibody (no. W6/32, BioLegend), at a concentration of $20 \mu \mathrm{g} \mathrm{ml}^{-1}$, was used to block MHC class I on REH and HPB-ALL cell lines transduced with HLA-A2.

T-cell activation assays. The reactivity of T-cell clones and TCR-transduced T cells was investigated by measurement of CD137 upregulation or IFN- $\gamma$ release. Briefly, 100,000 cells per well of indicated target cell lines or primary patient tumor cells were coincubated with T-cell clones or TCR-transduced PBMC (50,000 cells per well). Where indicated, target cells were pulsed with the specified concentrations of peptide for 1-2 h or electroporated with mRNA encoding full-length TdT, washed and then cocultured with effector cells. Following 14-16 h of coincubation, plates were centrifuged at $400 \mathrm{~g}$ for $3 \mathrm{~min}$. Culture supernatants were harvested for measurement of IFN- $\gamma$ by ELISA, and remaining cells were stained for flow cytometry to measure upregulation of CD137 on live CD8 ${ }^{+} \mathrm{T}$ cells. Results are reported either as percentage of $\mathrm{CD} 137^{+}$of $\mathrm{CD}^{+}$cells (labeled as $\mathrm{CD} 137^{+} / \mathrm{CD}^{+}$ cells) or percentage of $\mathrm{CD}_{137^{+}}$events among TCR-transduced CD8 ${ }^{+} \mathrm{T}$ cells (only for TCR-transduced patient T cells in Extended Data Fig. 8). In some experiments, cells were labeled with $0.75 \mu \mathrm{M}$ of the fluorescent cell staining dye CellTrace Violet (CTV, Life Technologies) or carboxyfluorescein succinimidyl ester (CFSE, Life Technologies) to distinguish between target and effector cells. The following reagents were acquired from BD Pharmingen or R\&D Systems: mouse anti-human IFN- $\gamma$ capture antibody (no. NIB42), Biotin Mouse Anti-Human IFN- $\gamma$ detection antibody (no. 4S.B3), streptavidin-HRP, stabilized tetramethylbenzidine and hydrogen peroxide as substrate solutions, sulfuric acid as stop solution and recombinant human IFN- $\gamma$ protein as standard. Assays were performed according to the manufacturers' instructions. Levels of IFN- $\gamma$ in serum harvested from mouse PB on day 2 after T-cell therapy were measured by Multiplexed Bead-Based Cytokine Assay from BD Pharmingen-the Human Th1/Th2/Th17 CBA Kit. The assay was preformed according to the manufacturer's instructions.

Proliferation assay. For the proliferation assay (Extended Data Fig. 4), 100,000 target cells were cocultured with 1G4, T1 or T3 TCR-transduced PBMCs for 5 days in T-cell medium at a 1/1 ratio in a round-bottom, 96-well plate in triplicate. To observe the presence or absence of proliferation for both $\mathrm{CD}^{+}$and $\mathrm{CD}^{+}$, transduced cells were labeled with $0.75 \mu \mathrm{M}$ of CSFE (Life Technologies). Following coculture, cells were harvested, washed and stained with human anti-CD3,-CD4, -CD8, -CD19, -CD10 and Live/Dead Fixable Near-IR to exclude dead cells. After $15 \mathrm{~min}$, cells were washed and resuspended in $200 \mu \mathrm{l}$ of FACS buffer containing 10,000 CountBright Absolute Counting Beads (ThermoFisher). Following data acquisition, an equal number of bead events $(3,000)$ was recorded from every well. Data are shown as histograms displaying CFSE levels for both $\mathrm{CD}^{+}$and $\mathrm{CD}^{+}$populations among $\mathrm{CD}^{+}, \mathrm{CD} 19^{-}, \mathrm{CD} 10^{-}$and Live/Dead Fixable Near-IR negative events, and as a percentage of the mean of live proliferating CD4 ${ }^{+}$ and $\mathrm{CD}^{+} \mathrm{T}$ cells acquired from three parallel wells.

Flow cytometry-based cytotoxicity assay using cell lines as targets. For cytotoxicity assays on B- and T-ALL cell lines, 50,000 target cells in T-cell medium were cocultured for $48 \mathrm{~h}$ with $\mathrm{T} 1$ or T3 TCR-transduced PBMCs in a round-bottom, 96-well plate in triplicate. Effector cells were defined as TCR-transduced CD8 ${ }^{+}$T cells in the PBMC product (routinely $>90 \%$ transduction efficiency and $55-65 \% \mathrm{CD}^{+} \mathrm{T}$ cells, the remainder being $\mathrm{CD} 4^{+} \mathrm{T}$ cells), with an $\mathrm{E} / \mathrm{T}$ ratio of $1 / 1$. Following coculture, cells were harvested, washed and stained with human anti-CD3, -CD8, -CD19 and Live/Dead Fixable Near-IR to exclude dead cells. After $15 \mathrm{~min}$, cells were washed and resuspended in $200 \mu \mathrm{l}$ of FACS buffer containing 10,000 CountBright Absolute Counting Beads (ThermoFisher) Following data acquisition, an equal number of bead events $(5,000)$ was recorded from every well. Data were normalized and reported as a percentage of the mean of live tumor cell numbers acquired from three parallel wells cocultured with mock-transduced T cells from the same donor. An example of the gating strategy used to identify live tumor cells is shown in Extended Data Fig. $4 \mathrm{f}$.

Flow cytometry-based assays for T-cell activation and cytotoxicity using primary human B- and T-ALL samples. Peripheral blood or BM samples from patients with B- and T-ALL were thawed and resuspended in T-cell medium containing low concentrations of IL-7 and IL-15 $\left(0.5 \mathrm{ng} \mathrm{ml}^{-1}\right)$. Cells were transferred to round-bottom, 96-well plates for assays measuring CD137 upregulation on TCR-transduced T cells or cytotoxicity on target cells. Individualized antibody panels and gating strategies used to identify malignant blasts and normal leukocyte populations were designed after review of diagnostic phenotyping available in hospital records. Allogeneic or, for patient no. $1 \mathrm{~N}$, autologous patient-derived, T cells transduced with TCRs were used in experiments. TCR-transduced cells were prelabeled with CTV dye to distinguish them from target cells. Wherever indicated, target cells were loaded with relevant peptides for 1-2 h, washed and then coincubated with TCR-transduced T cells for measurement of CD137 upregulation, as described above. For cytotoxicity assays, 50,000 target cells per well were coincubated with equal numbers of effector cells in two to four parallels per condition for $48-72 \mathrm{~h}$ and then stained with individualized antibody panels for flow cytometry. CountBright Absolute Counting Beads were utilized for acquisition of standardization, and data were normalized and reported as described above. For visual display of flow-cytometry plots, we utilized unsupervised nonlinear dimensionality reduction algorithms such as $t$-SNE using FlowJo (TreeStar) software.

Knockout of TdT in NALM-6 cells. CRISPR-Cas9-mediated knockout was done as previously described ${ }^{55}$ using the guide RNA 5'-ggc gct atg cca cac atg ag-3' to target the TdT epitope located at the end of exon 10 of the TdT gene. Following electroporation, modified NALM- 6 cells were enriched by FACS to generate a bulk culture showing heterogeneous TdT knockout (NALM-6- $\Delta$ TdT-bulk). A clone was generated by limited dilution that showed the same partial deletion of the target epitope (NALM-6- $\Delta \mathrm{TdT}_{475-481}$ ) in both alleles. To verify TdT knockout, genomic DNA was isolated from indicated cell lines (GeneJET Genomic DNA Purification Kit, ThermoFisher) and the targeted region was amplified by PCR (Phusion, ThermoFisher: sense, 5'-tca cta gag gga tgt agc cac c-3'; antisense, 5'-act cat tgc caa cac caa gg-3'), then PCR fragments were purified (Invisorb Fragment CleanUp, Stratec) and sent for sequencing (Eurofins Genomics) using the indicated PCR primers.

In vivo TdT TCR T-cell activity in two xenograft B-ALL cell line models. This work was approved by the Norwegian Food Safety Authority (application ID: 17500). All experiments were performed in compliance with the institutional guidelines and 2010/63/EU directive on the protection of animals used for scientific purposes. Male and female NOD-scid IL2Rg ${ }^{\text {null }}$ (NSG) mice, 8-10 weeks old and bred in-house were used in these experiments. On day 11 , mice were sublethally irradiated with 2.5-Gy radiation using a MultiRad225 X-ray irradiator (RPS services). Next, $4 \times 10^{6}$ or $1 \times 10^{6}$ cells of human B-ALL cell lines BV173 or NALM-6, retrovirally transduced to express GFP and firefly luciferase, were injected on day 10 through the tail vein. After leukemia was established and confirmed by BLI on day -1 , mice were treated with $10^{7} \mathrm{PBMC}$ transduced with either T1, T3 or a control TCR targeting NY-ESO-1 (1G4) ${ }^{19}$. A separate group of control mice did not receive any T-cell injections. To ensure T-cell survival, mice were injected intraperitoneally daily with 2,500 IU IL-2 (R\&D Systems) followed by BLI (IVIS Spectrum in vivo imaging system, and analysis by Living image software v.4.5.2, PerkinElmer), and blood analysis was performed by flow cytometry at different intervals. For survival analysis, mice were observed for clinical signs of tumor spread and were sacrificed if they developed $>20 \%$ weight loss, hunched posture, ruffled fur or limb paralysis. Experiments were terminated 2 months after T-cell injection to avoid graft-versus-host disease, and surviving mice in treated groups were humanely sacrificed. In two experimental cohorts (BV173 or NALM6), BM from surviving T3-treated mice at the end of experiment (day 57 or 60), or from 1G4-treated or untreated mice sacrificed due to high leukemia burden, was harvested and processed for flow cytometry to determine the presence of T cells and tumor cells, and expression of TdT and HLA-A2.

In vivo TdT TCR T-cell activity in a patient-derived xenograft model. Experiments were performed according to the guidelines and permissions obtained from the ethics committees at Stockholm Norra Djurförsöksetisks Nämd (no. 17978-2018). Experimental mice were housed at two to five per cage in IVC-Mouse GM 500 cages with a light cycle of $06.00-18.00,21^{\circ} \mathrm{C}$ and $50 \%$ humidity. Female NOD.Cg-Prkdc ${ }^{\text {scid }} \mathrm{Il} 2 \mathrm{rg}^{\mathrm{tm} 1 \mathrm{Wjl}_{\mathrm{j}}} / \mathrm{SzJ}$ mice (NSG; Jackson Laboratory, no. 005557) 9-15 weeks of age were sublethally irradiated with two doses of $1.65 \mathrm{~Gy}$ (X-ray source) $4 \mathrm{~h}$ apart. Viable, T-cell-depleted BM cells from HLA-A $2^{\text {pos }}$ B-ALL patient no. $20 \mathrm{O}$ were yield sorted on a BD FACS Aria Fusion by exclusion of $7-\mathrm{AAD}^{+}$and $\mathrm{CD}^{+}$cells, and $4 \times 10^{5}$ cells were injected via the tail vein of NSG mice $4-6 \mathrm{~h}$ after the final irradiation dose. Stable engraftment was confirmed by $\mathrm{PB}$ analysis and BM aspiration from all transplanted mice 18-19 and 20-26 days after transplantation, respectively. NSG mice were allocated into untreated, DMF5 and T3 T-cell groups based on their engraftment levels so that mean human leukemic engraftment was comparable among groups. Then, $7.5 \times 10^{6}$ mTCR $-\beta^{+}$CD $8^{+}$T cells transduced with DMF5 TCR or T3 TCR were injected 22-25 days after transplantation of primary B-ALL cells, and all groups received a daily intraperitoneal injection of 2,500 IU of IL-2 (R\&D Systems) per mouse. Engraftment was monitored in PB 3 and 10 days after T-cell infusion. Following euthanization of the mice 11 days after T-cell infusion, BM, spleen and $\mathrm{PB}$ were subjected to detailed flow-cytometry analysis to identify leukemic cells and infused T cells. For analysis of BM samples, a minimum of $1.8 \times 10^{5}$ events were acquired for all mice and at least $3 \times 10^{5}$ events were acquired for all but two T3-cell-treated mice to determine MRD levels according to NOPHO guidelines.

Cell counting of BM (two tibiae, two femora and two crista) and spleen from euthanized mice was performed using a Sysmex hematologic cell counter. TrueCount beads (BD Biosciences) were added to whole PB according to the manufacturer's instructions and stained for mouse CD45.1 and human CD45 to determine absolute MNC counts per microliter of blood. Leukemia burden and 
TCR-transduced CD8 ${ }^{+}$cells were quantified for each tissue based on the frequency of human $\mathrm{CD} 45^{+} \mathrm{CD} 19^{+} \mathrm{CD} 10^{+}$and human $\mathrm{CD} 45^{+} \mathrm{CD} 3^{+} \mathrm{CD} 8^{+} \mathrm{mTCR}-\beta^{+}$cells, respectively, in relation to total cell count.

\section{In vivo impact of T3-cell treatment on normal human hematopoiesis in} humanized NSG mice. Experiments were performed according to the guidelines and obtained permissions from the ethics committees at Stockholm Norra Djurförsöksetisks Nämd (no. 17978-2018). Experimental mice were housed at two to five per cage in IVC-Mouse GM 500 cages with a light cycle of 04.00-16.00, $21^{\circ} \mathrm{C}$ and $50 \%$ humidity. Female NSG mice stably engrafted with HLA-A2 ${ }^{\text {pos }}$ human cord blood cells were purchased from the Jackson Laboratory. Following confirmation of human engraftment 24 weeks after transplantation, single-cell suspensions from spleens of three engrafted NSG mice were transduced with 1G4 or T3 TCR constructs and expanded as described above. Activity of NSG-derived $1 \mathrm{G} 4$ and T3 cells was validated in vitro by performing flow-cytometry-based cytotoxicity assay on BV173 cells, in parallel with infusion of $10 \times 10^{6} \mathrm{~T}$ cells into the remaining humanized NSG mice through tail vein injection. Because engrafted mice also contained endogenous cord-blood-derived T cells with autocrine IL-2 production potential, supportive daily intraperitoneal infusion of $500 \mathrm{IU}$ of IL-2 was not included for half of the mice (since no differences were observed with or without IL-2 supportive infusions, the data from these groups were pooled). The persistence of infused T cells was monitored in $\mathrm{PB}$, and the impact of therapy on mature blood cell lineages was monitored in PB, spleen, thymus and BM by flow cytometry 17 days after T-cell infusion. The impact on human T-cell progenitors in mouse thymus was investigated through surface and intracellular FACS staining. Cell counting of BM (two tibiae, two femora and two crista) from terminated mice was performed using a Sysmex hematologic cell counter.

In vitro impact on clonogenic potential of normal hematopoietic progenitors. Bone marrow MNCs were obtained from four HLA-A2 ${ }^{\text {pos }}$ healthy donors collected at Karolinska University Hospital, with informed consent and ethical approval (no. EPN 2018/901-31). Two hundred and fifty viable single CD $34^{+}$progenitor cells, identified by DAPI and mature lineage exclusion, were sorted on a BD FACS Aria Fusion and cocultured with $500 \mathrm{CD}^{-} \mathrm{CD} 19^{-}$(sorted on BD FACS Aria Fusion) 1G4, T1 or T3 TCR-transduced T cells in StemSpan SFEM (StemCell Technologies), supplemented with 10\% BIT9500 (StemCell Technologies), penicillin/streptavidin ( $100 \mathrm{U} \mathrm{ml}^{-1}$; Hyclone Laboratories), $2-\beta$-mercaptoethanol (2-ME, $0.1 \mathrm{mM}$; Sigma-Aldrich), stem cell factor (SCF, $\left.10 \mathrm{ng} \mathrm{ml}^{-1}\right)$, flt 3 ligand (FL, $10 \mathrm{ng} \mathrm{ml}^{-1}$ ), thrombopoietin (TPO, $10 \mathrm{ng} \mathrm{ml}^{-1}$ ), interleukin-3 (IL-3, $5 \mathrm{ng} \mathrm{ml}^{-1}$ ), granulocyte colony-stimulating factor (G-CSF, $10 \mathrm{ng} \mathrm{ml}^{-1}$ ), granulocyte macrophage colony-stimulating factor (GM-CSF, $10 \mathrm{ng} \mathrm{ml}^{-1}$ ) and erythropoietin (EPO, $1 \mathrm{U} \mathrm{ml}^{-1}$ ) in $37^{\circ} \mathrm{C}, 5 \% \mathrm{CO}_{2}$. $\mathrm{CD} 4^{+}$lin $^{-}$progenitors cultured without $\mathrm{T}$ cells were used as controls. After $72 \mathrm{~h}$, cells from the cocultures were transferred to cytokine-containing methylcellulose (MethoCult, no. H4434, StemCell Technologies) in Iscove's modified Dulbecco's Medium (Gibco) supplemented with $20 \%$ fetal bovine serum (Sigma-Aldrich), L-glutamine ( $2 \mathrm{mM}$, Sigma-Aldrich), penicillin/streptavidin $\left(100 \mathrm{U} \mathrm{ml}^{-1}\right)$ and $2-\mathrm{ME}(0.1 \mathrm{mM})$, to facilitate colony generation. After 14 days in methylcellulose, colonies were scored under an inverted microscope as myeloid or erythroid. As a positive control, CD34 $4^{+}$inn $^{-}$ progenitors were externally loaded with $1 \mu \mathrm{M}$ peptide- 1 or peptide- 3 in StemSpan SFEM for $2 \mathrm{~h}$, followed by $48 \mathrm{~h}$ of coculture with or without transduced T cells in the presence of $100 \mathrm{nM}$ peptides, and colonies were scored 10 days after transfer of cells to methylcellulose as described above.

Peptide-HLA-A2 model generation. To generate models, a list of existing peptide-HLA-A2 structures from Protein Data Bank (PDB) was compiled. More than ten HLA-A2 structures were solved presenting nonameric peptides. We set out to use a high-resolution model (PDB accession code 5MEQ) ${ }^{56}$ in which HLA-A2 presents a peptide (ILAKFLHTL) from human telomerase reverse transcriptase. For the peptide-3HLA-A2 model, our options were much more limited since considerably fewer HLA-A2 structures were solved presenting undecamer peptides. We used a high-resolution model (PDB accession code 5D9S $)^{57}$ in which HLA-A2 presents an 11-mer peptide (FVLELEPEWTV) derived from Toxoplasma gondii. All peptide residues presented by HLA-A2 from these two models were replaced using pymol mutagenesis, and residues with minimum clashes with HLA-A2 were selected for generation of the peptide-1- and peptide-3-HLA-A2 models.

Statistical analysis. Statistical analysis was performed in GraphPad Prism v.6-8 (GraphPad Software). To compare more than two experimental groups, ordinary one-way analysis of variance (ANOVA) test with adjustment for multiple comparisons with Tukey's post-test was employed. Survival analysis was performed using log-rank (Mantel-Cox) test. To determine differences between in vivo treatment groups in the PDX and humanized NSG mouse models, Kruskal-Wallis ANOVA by Dunn's multiple comparisons test and two-tailed Mann-Whitney test were performed. $P<0.05$ was considered statistically significant.

Reporting Summary. Further information on research design is available in the Nature Research Reporting Summary linked to this article.

\section{Data availability}

The data that support the findings of this study are included in the manuscript and in the Supplementary information. Additional datasets used in the study are: PDB (accession codes: 5MEQ (https://www.rcsb.org/structure/5MEQ) and 5D9S (ttps://www.rcsb.org/structure/5D9S); Homo sapiens canonical database, using the Mascot search engine v.2.2.04 (www.matrixscience.com); Uniprot Homo sapiens database, using Mascot v.2.2.07 (www.matrixscience.com); curated human proteome databases UniProtKB/Swiss-Prot and PDB by ScanProsite tool (https:// prosite.expasy.org/scanprosite/); and peptide-MHC class I binding prediction algorithm NetMHC v.4.0 (http://www.cbs.dtu.dk/services/NetMHC/). Source data are provided with this paper.

\section{References}

48. Tosato, G. \& Cohen, J. I. Generation of Epstein-Barr Virus (EBV)-immortalized B cell lines. Curr. Protoc. Immunol. Chapter 7, Unit 7.22 (2007)

49. Toebes, M. et al. Design and use of conditional MHC class I ligands. Nat. Med. 12, 246-251 (2006).

50. Hadrup, S. R. et al. Parallel detection of antigen-specific T-cell responses by multidimensional encoding of MHC multimers. Nat. Methods $\mathbf{6}$, 520-526 (2009)

51. Scheper, W. et al. Low and variable tumor reactivity of the intratumoral TCR repertoire in human cancers. Nat. Med. 25, 89-94 (2019).

52. Stronen, E. et al. Targeting of cancer neoantigens with donor-derived $\mathrm{T}$ cell receptor repertoires. Science 352, 1337-1341 (2016).

53. Linnemann, C. et al. High-throughput identification of antigen-specific TCRs by TCR gene capture. Nat. Med. 19, 1534-1541 (2013).

54. Brochet, X., Lefranc, M. P. \& Giudicelli, V. IMGT/V-QUEST: the highly customized and integrated system for IG and TR standardized V-J and V-D-J sequence analysis. Nucleic Acids Res. 36, W503-W508 (2008).

55. Ran, F. A. et al. Genome engineering using the CRISPR-Cas9 system. Nat. Protoc. 8, 2281-2308 (2013).

56. Cole, D. K. et al. Structural mechanism underpinning cross-reactivity of a $\mathrm{CD}^{+} \mathrm{T}$-cell clone that recognizes a peptide derived from human telomerase reverse transcriptase. J. Biol. Chem. 292, 802-813 (2017).

57. McMurtrey, C. et al. Toxoplasma gondii peptide ligands open the gate of the HLA class I binding groove. eLife 5, e12556 (2016).

\section{Acknowledgements}

We thank Oslo University Hospital flow cytometry core facility for excellent technical assistance, and M. Saare and S. T. Flåm for isolation of human thymocytes. This work was supported by South-Eastern Regional Health Authority Norway; the Research Council of Norway; the Norwegian Cancer Society; the Norwegian Childhood Cancer Foundation; Stiftelsen Kristian Gerhard Jebsen; European Research Council under the European Union's Horizon 2020 research and innovation program (grant agreement no. 865805); the University of Oslo and Oslo University Hospital and Novo Nordisk Foundation (to J. O.); the Knut and Alice Wallenberg Foundation (nos. KAW 2016.0105 to S.E.W.J. and KAW 2015.0195 to P.W.); the Tobias Foundation (no. 4-1122/2014 to S.E.W.J.); the Center for Innovative Medicine at Karolinska Institutet (no. 613/06 to S.E.W.J.); The Swedish Research Council (nos. 538-2013-8995 to S.E.W.J. and 2015-03561 to P.W.); The UK Medical Research Council (no. MC_UU_12009/5 to S.E.W.J.); and the National Institutes of Health (no. RO1-AI103867-08 to K.C.G.).

\section{Author contributions}

M.A. conceived ideas for study design. M.A., E.G., Y.L., M. Lehander, S.V.C., W.Y., C.K., M.C.O. and M. Leisegang designed and performed experiments, analyzed data and wrote the manuscript. Z.F., M.-L.B., A.H. and S.M. performed experimental work and analyzed data. X.Y. and K.C.G. generated peptide-MHC models and wrote the manuscript. R.C.B., A.H.R., G.M.C.J. and P.A.V. generated and analyzed MS data and wrote the manuscript. G.E.T., M.G., S.L., L.T.O., B.A.L. and J.B. provided clinical samples and/or data. J.B. wrote the manuscript. E.T., E.S., M.T. and A.K. participated in the design of experiments and provided advice and support. T.N.S. and E.S. reviewed and wrote the manuscript. S.E.W.J. and P.W. contributed to experimental design, analysis of experiments and review and writing of the manuscript. J.O. was the principal investigator of the study and conceived the idea, designed, analyzed and supervised the study and wrote the manuscript.

\section{Competing interests}

J.O. has a research collaboration with Kite Pharma/Gilead, Inc., and a patent application by the institutional technology transfer office Inven2 was filed (patent application number 2113437.4) that covers TCR receptor sequences (inventors J.O. and M.A.) T.N.S. is a consultant for Adaptive Biotechnologies, AIMM Therapeutics, Allogene Therapeutics, Merus, Neogene Therapeutics, Neon Therapeutics and Scenic Biotech; is a recipient of grant/research support from MSD, Bristol-Myers Squibb and Merck $\mathrm{KGaA}$; is a stockholder in AIMM Therapeutics, Allogene Therapeutics, Merus, Neogene Therapeutics and Neon Therapeutics; and is venture partner at Third Rock Ventures. 
G.E.T. has received research support from Mundipharma and lecture honoraria from Mundipharma, Abbvie, Janssen-Cilag, Alexion Pharmaceuticals and Roche Norway. The remaining authors declare no competing interests.

\section{Additional information}

Extended data is available for this paper at https://doi.org/10.1038/s41587-021-01089-x.
Supplementary information The online version contains supplementary material available at https://doi.org/10.1038/s41587-021-01089-x.

Correspondence and requests for materials should be addressed to Johanna Olweus. Peer review information Nature Biotechnology thanks Sebastian Kobold, Sara Mastaglio and the other, anonymous, reviewer(s) for their contribution to the peer review of this work

Reprints and permissions information is available at www.nature.com/reprints. 
$\mathbf{a}$

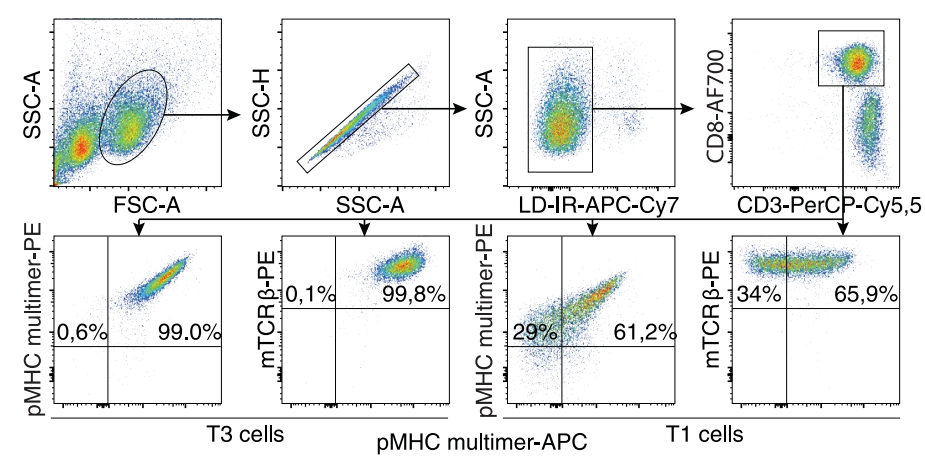

b
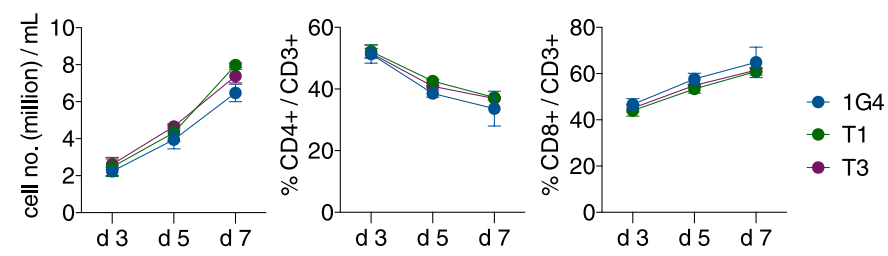

C

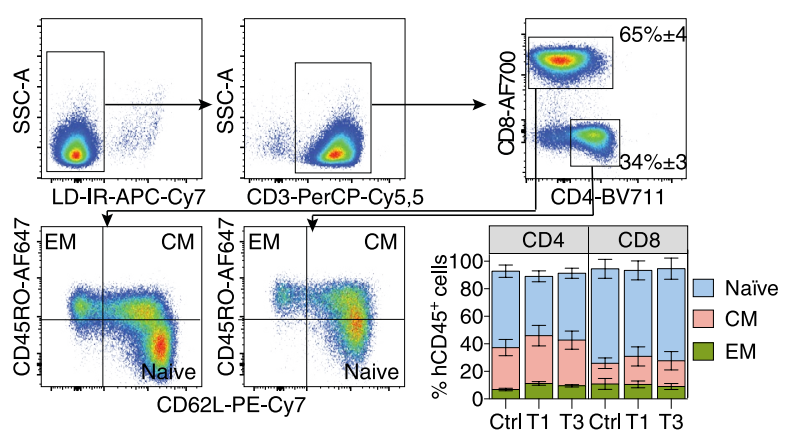

d

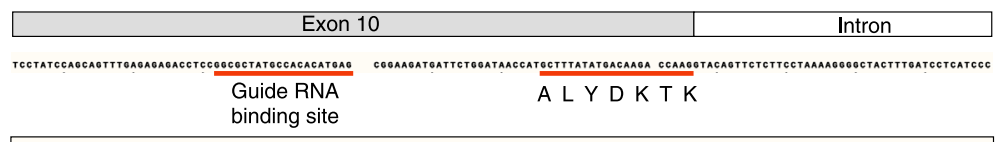

NALM-6

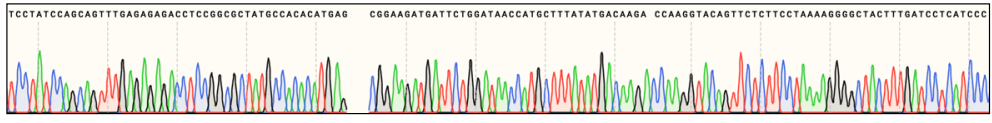

NALM-6-

$\triangle \mathrm{TdT}_{475-48}$

(Clone)

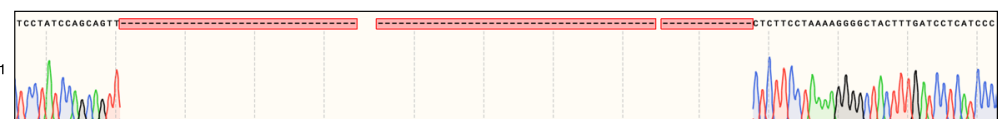

e

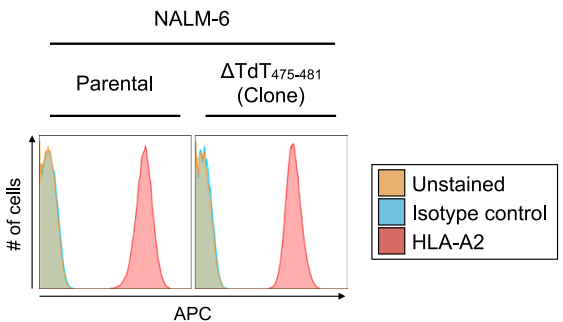

Extended Data Fig. 1 | T1 and T3 cells maintain a predominantly naïve/CM phenotype following expansion. (a) Gating strategy to analyze TCR transduced T cells (percentage of parental gate is shown). Top four panels: gating on FSC/SSChigh, Live/Dead Fixable Near-IR ${ }^{\text {neg, }}$ CD8 ${ }^{+}$singlets. Lower four panels: cells staining positively for APC and PE-labeled pMHC multimers complexed with either peptide-3 (left two plots: T3-transduced cells) or peptide-1 (right two plots: T1-transduced cells) and anti-mouse TCR- $\beta$ antibody. Although $>90 \%$ of T1 cells stain positively for the pMHC-PE multimer, a lower \% was positive for the corresponding APC multimer due to lower fluorescence intensity of APC relative to PE. (b) Expansion of PB T cells after transduction with T1, T3 or $1 \mathrm{G} 4$ at indicated days after retroviral transduction. Data shown as mean \pm s.e.m. of $n=3\left(\mathrm{~d} 3\right.$ and d7) or $n=2(\mathrm{~d} 5) \mathrm{HLA}-\mathrm{A} 2^{\text {pos }}$ donors. (c) FACS plots showing phenotyping of T cells transduced with T1 on day 7 after spinoculation, gating on FSC/SSChigh, Live/Dead Fixable Near-IR neg, $\mathrm{CD}^{+}$singlets, that are either $\mathrm{CD}^{+}$or $\mathrm{CD} 8^{+}$. Naïve, $\mathrm{CM}$ (central memory) and EM (effector memory) T cells. Bar graph in the lower right corner shows proportion of naïve, $\mathrm{CM}$ and $\mathrm{EM} \mathrm{CD} 4^{+}$or $\mathrm{CD} 8^{+} \mathrm{T}$ cells on $\mathrm{d} 7$ after retroviral transduction with $\mathrm{T} 1, \mathrm{~T} 3$ or control TCR. Inset numbers in the top right plot represent mean \pm s.e.m. of all groups analyzed together due to similar proportions of $C D 8^{+}$and $C D 4^{+} T$ cells in experimental groups. Data shown as mean \pm s.e.m. of $n=3$ donors. (d) Top: The region of gDNA in exon 10 which ends with the first 7 amino acids of the A2-presented epitope, selected as binding site for guide RNA. Middle: sequencing of gDNA from wild type NALM-6 cells, in which the TdT sequence corresponds to the sequence deposited in the database. Bottom: after cloning of CRISPR/Cas9-modified NALM- 6 cultures, the selected clone shows a clean deletion in both alleles of the TdT gene, including the first 7 amino acids of the A2-presented epitope. (e) HLA-A2 expression on NALM-6 cells with TdT knock out (right) is unaffected relative to parental cell line (left). 
a

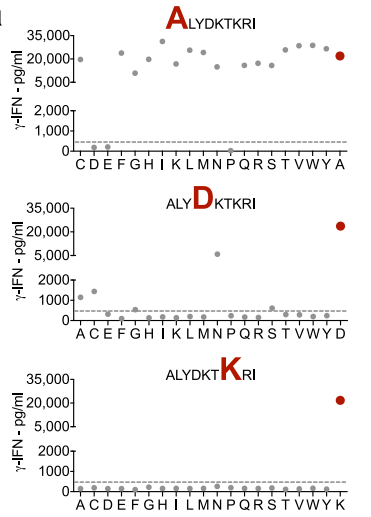

b

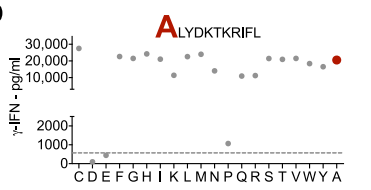

ALY $D_{\text {KTKRIFL }}$
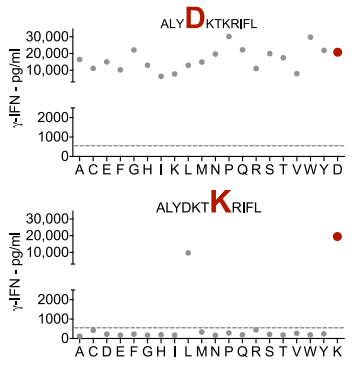

${ }_{\text {ALYDKTKRI }} \mathbf{F}_{\mathrm{L}}$

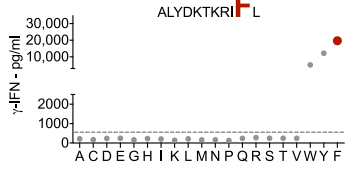

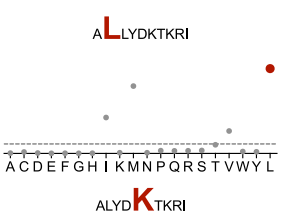

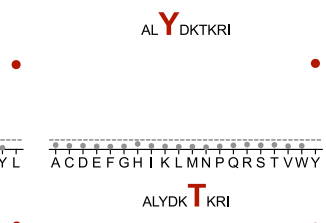

.

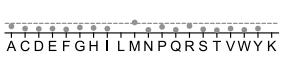

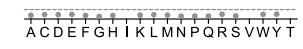
ALYKKTKR

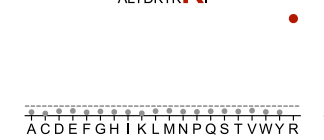

ALYDKTKR I .

\section{ACDEFGHKLMNQRSTVWYY}
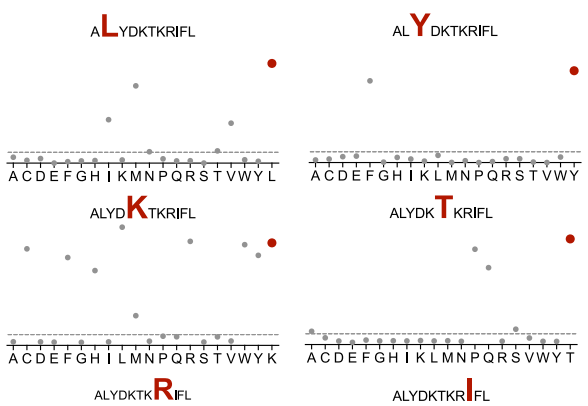

ALYDKTKR $I_{F L}$
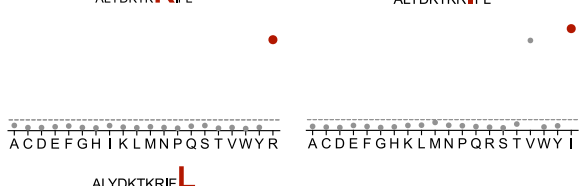
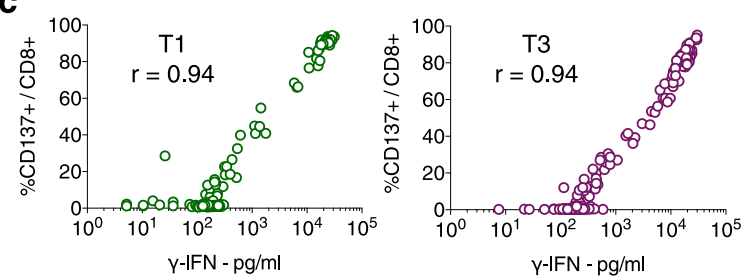

d

$$
\begin{aligned}
& \mathrm{T} 1=\mathrm{X} \text { [LIMV] Y [DACGNS] [KLM] [TIV] K R [ILMV] } \\
& \mathrm{T3}=\mathrm{X} \text { [LIMNTV] [YF]X[KCFHLMRWY] [TAPQS][KL] R } \\
& {[\text { IV] [FWY] [LGHIKPSTV] }}
\end{aligned}
$$

e

E5RII4_HUMAN Small integral membrane protein 19 (SMIM19):

MAGGYGVMGDDGSIDYTVHEAWNEATNVYLIVILVSF GLFMYAKRIFGFWRIEENHNGGTSLCCVKWMCSATF LTASYFLVKERIL

SMIM19 peptide $=$ GLFMYAKRIFG

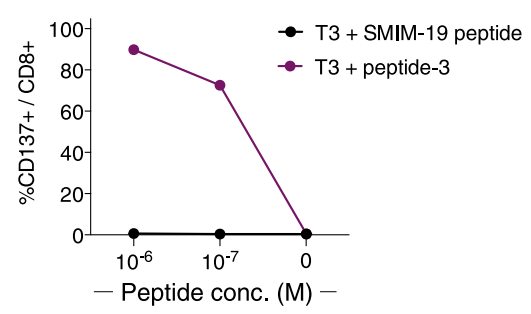

Extended Data Fig. 2 | Comprehensive mapping of peptide reactivity does not reveal naturally existing peptides to which T1 or T3 cells cross-react. $(a, b)$ Graphs depicting IFN- $\gamma$ response of T1 (a) and T3 (b) cells to EBV-LCLs loaded with individual peptides from mimotope libraries containing a total of 161 9-mers for peptide-1 and 20111-mers for peptide-3, at a concentration of ( $\left.2 \times 10^{-7} \mathrm{M}\right)$. Red dot in each graph represents response to wild-type peptide. Substituted amino acid in the original peptide is highlighted in red in the graph heading. IFN- $\gamma$ concentration range for positive reactions was 500 - $31254 \mathrm{pg} / \mathrm{mL}$ (cut-off indicated by horizontal lines, one replicate per condition). (c) Correlation analysis between data for IFN- $\gamma$ release, and percentage of cells expressing CD137, by T1 (left) and T3 cells (right) following activation by EBV-LCLs loaded with individual peptides from the mimotope libraries. $r=$ Pearson correlation coefficient. (d) Peptide reactivity motifs for T1 and T3 that were queried in the ScanProsite search tool against human proteome databases. $X$ indicates that any amino acid is allowed, while amino acids in square brackets [] indicate alternatives that are allowed for that given position in the peptide motifs. (e) Amino acid sequence of the human small integral membrane protein 19 (SMIM19), as listed in the non-curated TrEMBL database. (f) Percentage CD137+ events among T3 CD8 ${ }^{+} \mathrm{T}$ cells after $18 \mathrm{~h}$ co-culture with target cells loaded with indicated concentrations of either peptide-3 or SMIM19 peptide. Data are shown as mean of three technical replicates from one experiment representative of 2 performed. 
a

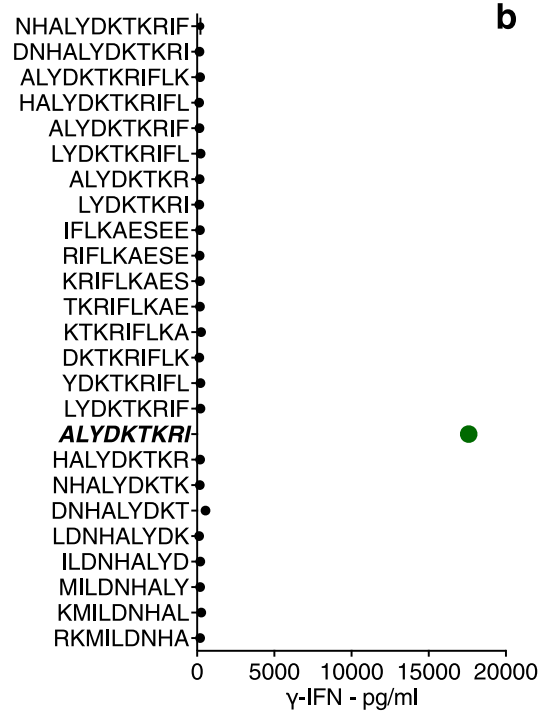

d

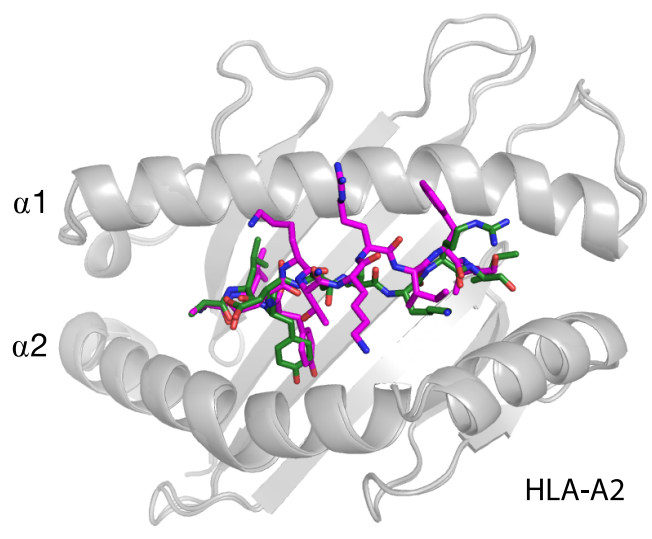

C
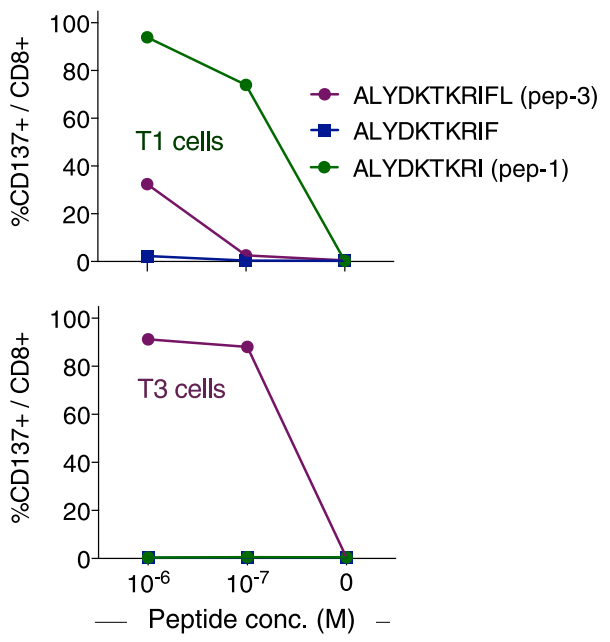

500010000150002000025000 $\gamma$-IFN pg/ml
Pep-1 : ALYDKTKRI

Pep-3 : ALYDKTKRIFL

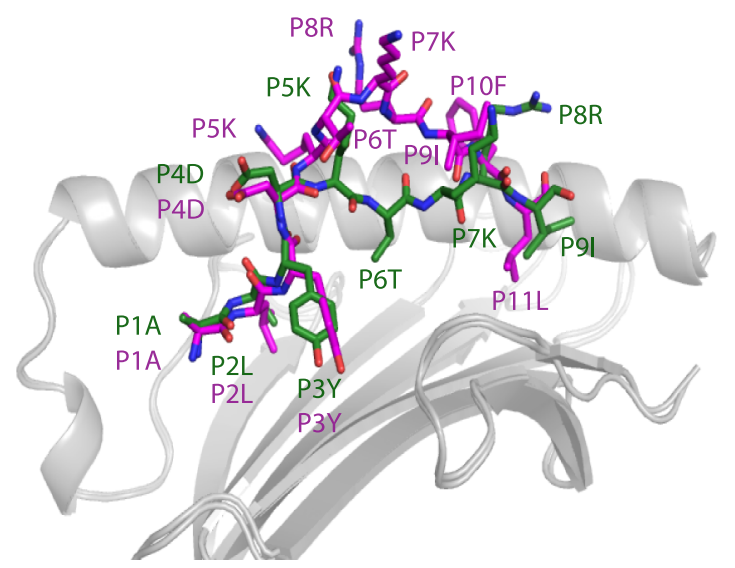

Extended Data Fig. 3 | T1 and T3 cell activation in response to length variants of cognate peptides and model of structural conformation of TdT peptides in the HLA-A2 peptide-binding groove. (a,b) IFN- $\gamma$ production by the $\mathrm{T} 1$ (a) and T3 (b) cells after co-culture with target cells loaded with indicated peptides. 9-mer and 11-mer peptides were included that contained amino acids upstream or downstream of the wild type peptide-1 and -3 in the TdT protein sequence. In addition, 8 to 12 amino acid long peptides were included that contained parts or all of peptide- 1 and -3. One replicate per condition. (c) Percentage of CD137+ events among T1 and T3 cells after co-culture with EBV-LCLs loaded with indicated concentrations of cognate, or non-cognate, peptide-1 (ALYDKTKRI) or -3 (ALYDKTKRIFL). Reactivity to a 10-mer peptide (ALYDKTKRIF) is also shown. One replicate per condition. Data shown are from one experiment representative of 2 performed. (d) Overlaid structural conformations of peptide-1 and -3 bound to the HLA-A2 molecule shown from top (left) and side view (right). HLA-A2 is shown as cartoon in gray. Peptide-1 (green) and peptide-3 (purple) are shown as stick. Individual amino acids are labeled as a number in the corresponding peptide followed by their symbol. The modeled peptide-1 on HLA-A2 displays a flattened conformation, and amino acids at position (P)4, P5, P7 and P8 are facing up for potential TCR engagement. The model is supported by the mimotope reactivity pattern, as T1 was intolerant to mutations in P4, P5, P7 and P8. Although P3Y is not facing upward for TCR contact, it is likely to stabilize the peptide-MHC interaction and is thus sensitive to mutations. The P4, P5, P7 and P8 as core TCR contact residues are salient features for 9-mer peptides presented by HLA-A2. Peptide-3 displays a bulged conformation, in which P4, P5, P6, P7, P8, P9 residues are facing upwards for TCR contact. P4D and P5K are less important for TCR engagement than for peptide-1, as P4D can be substituted by almost any amino acid and P5K can be substituted by amino acids with long or bulky side chains. P6-P10 are potentially important for TCR contact, and as shown in our cross-reactivity assay, T3 was less tolerant for substitutions of these amino acids. 
a

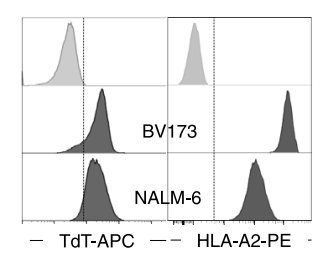

b

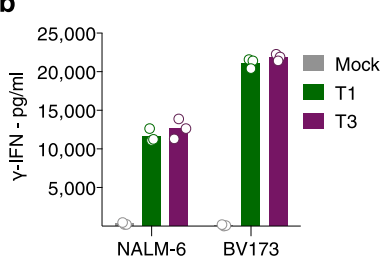

c

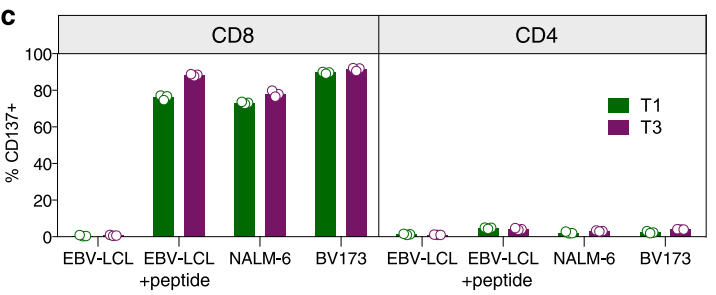

d

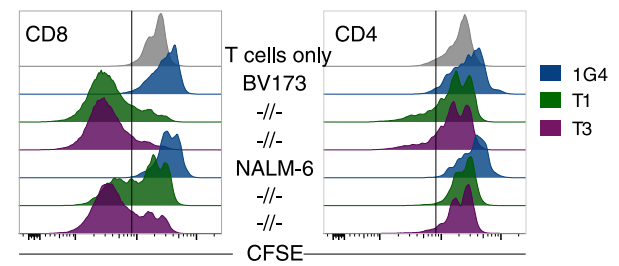

f
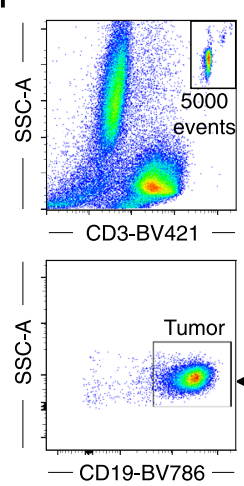
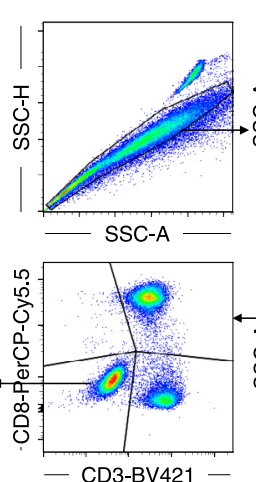
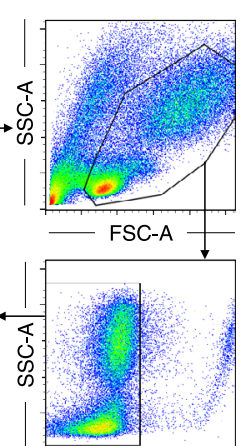

LD-IR-APC-Cy7 - e

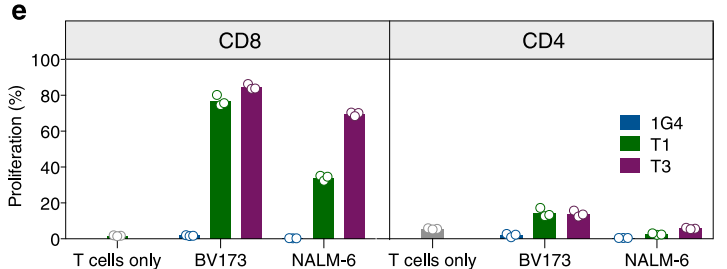

g

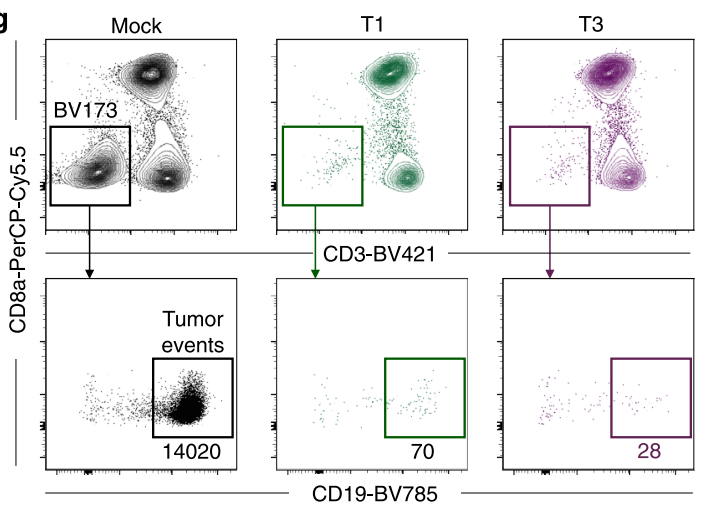

Extended Data Fig. 4 | T1 and T3 cells are activated by, and effectively kill, TdTpos HLA-A2pos leukemia cell lines. (a) Flow cytometry histograms showing natural TdT or HLA-A2 expression in the B-ALL cell lines NALM- 6 and BV173. (b) IFN- $\gamma$ production in the co-culture supernatants of T1, T3 or mock T cells with indicated cell lines as measured by ELISA. (c) Expression of CD137 measured by flow cytometry by T1 and T3 cells after $18 \mathrm{~h}$ co-culture with indicated cell lines, loaded or not with relevant peptide and gated on CD8 ${ }^{+}$or CD4+ ${ }^{+}$cells. (d,e) Proliferation of PB T cells following transduction of T1, T3 or 1G4 in response to NALM-6 and BV173 following 5 days of co-culture at an E/T ratio of 1/1. Percentage proliferating cells is calculated based on events with low Cell Trace Violet staining out of total events that are FSC/SSChigh, Live/Dead Fixable Near-IR ${ }^{\text {neg }}$, CD3 ${ }^{+}$singlets staining positively for either $\mathrm{CD} 8^{+}$or $\mathrm{CD}^{+}$. Bar graphs in (b), (c) and (e) show mean of three technical replicates from one experiment representative of 2 or 3 performed. ( $f$ ) Gating strategy for the flow cytometry-based killing assay to enumerate BV173 tumor cells, here co-cultured with mock-transduced cells. Fluorescent beads $(10,000)$ were added into each well and 5,000 beads were acquired for flow cytometry analysis. Live BV173 tumor cells were identified as Live/Dead Fixable Near-IR ${ }^{\text {neg }}$ singlet cells that were $\mathrm{CD}^{-}, \mathrm{CD}^{-}$and $\mathrm{CD}^{+}{ }^{+}$. (g) Flow cytometry plots of BV173 cells co-cultured with mock (black), $\mathrm{T} 1$ (green) and T3 cells (purple) for $48 \mathrm{~h}$. Inset numbers display event counts within the live tumor cell gate. 
a

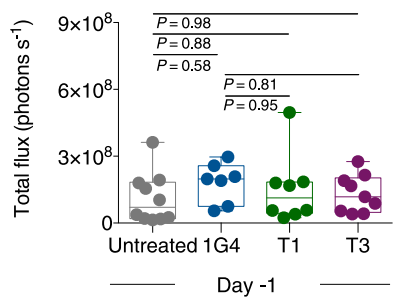

e

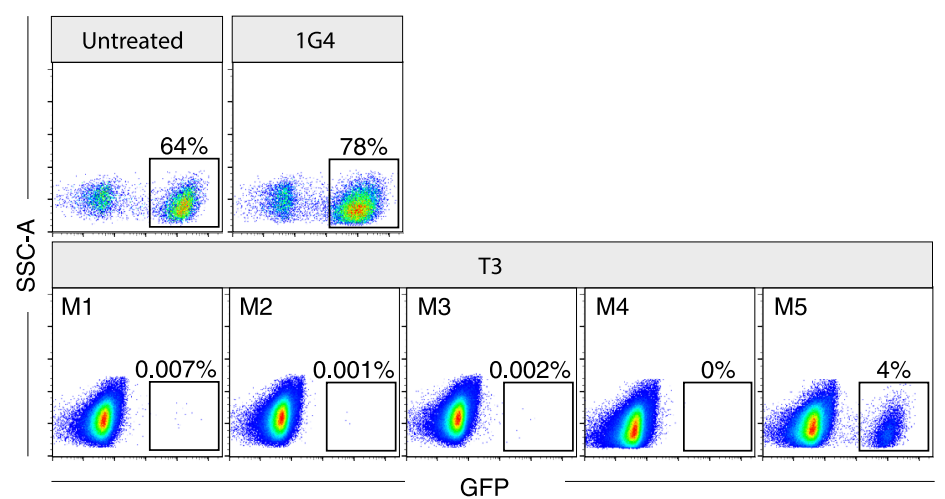

b

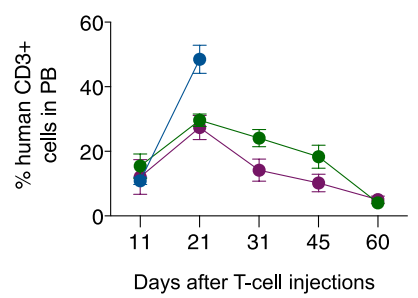

Days after T-cell injections
C

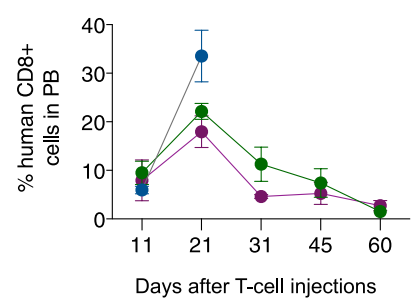

d

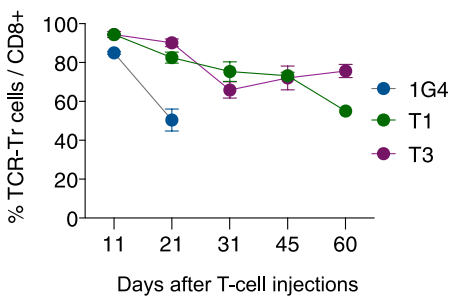

f

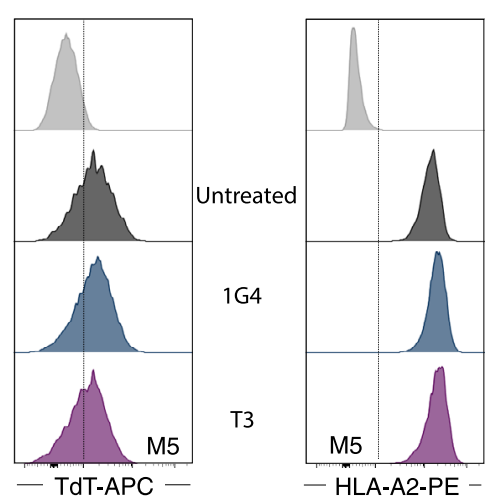

g

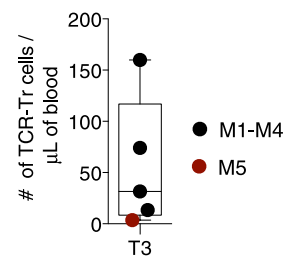

Extended Data Fig. 5 | T1 and T3 cells proliferate and control BV173 ALL in vivo. (a) Bioluminescence imaging (BLI) analysis of NSG mice on day 9 after BV173 $3^{\text {ffluc-eGFP }}$ cell injection, one day prior to T-cell therapy. Data were pooled from two independent experiments, untreated $n=10,1 G 4 n=7, T 1 n=8, T 3$ $n=9$. Data analysis by one-way ANOVA with Tukey's multiple comparison test. $(b, c)$ The percentage of human $C D 3^{+}(b)$ and $C D 8^{+}$cells $(c)$ in PB of mice analyzed at indicated time points out of total CD45+ human and CD45+ mice leukocytes. (d) Percent cells expressing T1, T3 and 1G4 TCRs among human $\mathrm{CD}^{+} \mathrm{T}$ cells throughout the experiment. Data in b-d are shown as mean \pm s.e.m., $n=5 /$ group. (e) Flow cytometry plots showing bone-marrow tumor burden in five T3-treated mice analysed on day 60 (M1-M5), compared to the 1G4-treated or untreated mouse at time of sacrifice (day 21). Threshold for positive leukemia detection was set as GFP+ cells $\geq 0.01 \%$ of live viable cells. (f) Expression of HLA-A2 and TdT in leukemia cells harvested from the bone marrow of untreated, 1G4 and the one T3-treated mouse with detectable tumor burden (M5). Light gray histograms represent negative control. (g) Numbers of TCR-transduced CD8+ cells in blood of T3-treated mice on day 60 ( $n=5$, M1-4: black circles, M5: red circle). Box plots in (a) and (g) show interquartile range $\left(25^{\text {th }}\right.$ to $75^{\text {th }}$ percentile) with central bar indicating the median and whiskers indicating the range while dots represent data from individual mice. 


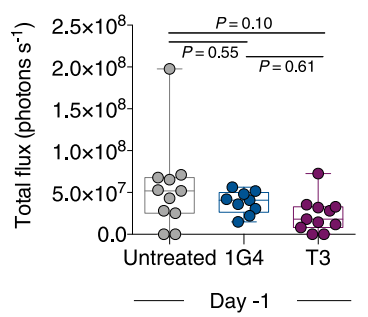

b

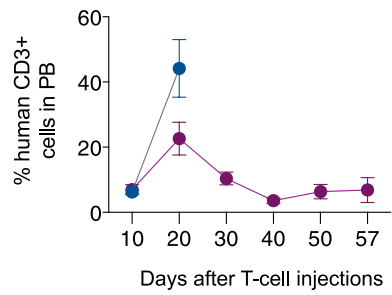

C

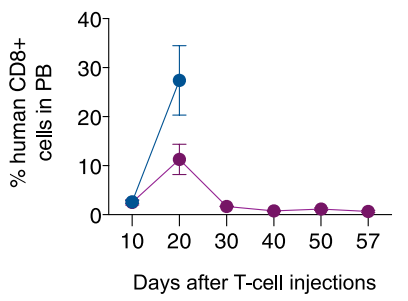

d

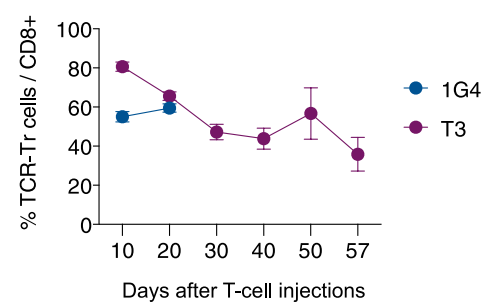

e

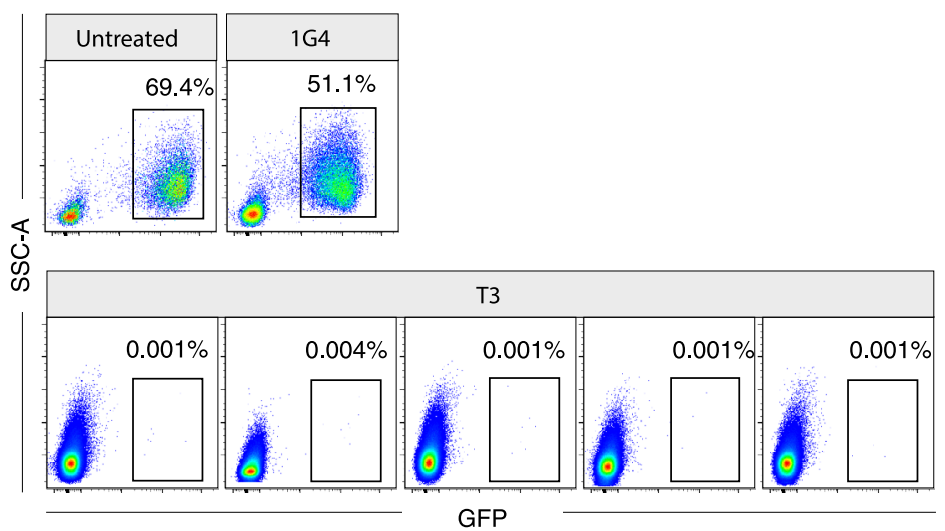

f
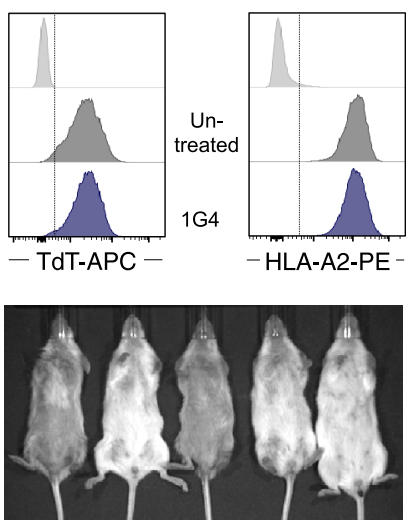

h
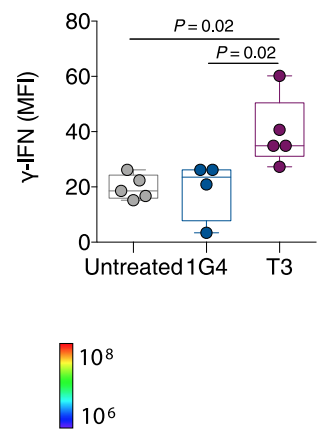

Total flux $\left(\right.$ photon $\mathrm{s}^{-1}$ )

Extended Data Fig. 6 | T3 cells proliferate and control NALM6 ALL in vivo. (a) BLI analysis of NSG mice on day 9 after NALM-6ffluc-eGFP cell injection, one day prior to T-cell therapy. Data were pooled from two independent experiments, untreated $n=11,1 G 4 n=9$, T3 $n=11$. Data analysis by one-way ANOVA with Tukey's multiple comparison test. (b,c) The percentage of human $\mathrm{CD}^{+}(\mathrm{b})$ and $\mathrm{CD} 8^{+}$cells $(\mathrm{c})$ in peripheral blood (PB) of mice analyzed at indicated time points out of total CD45+ human and CD45+ mouse leukocytes. (d) Percent cells expressing T3 and 1G4 TCRs among human CD8 ${ }^{+} \mathrm{T}$ cells throughout the experiment. Data in b-d are shown as mean \pm s.e.m., $n=5$ (1G4) and $n=6$ (T3). (e) Flow cytometry plots showing bone-marrow tumor burden in one untreated and one 1G4-treated mice at time of sacrifice (day 14), and in five T3-treated mice sacrificed at end of experiment (day 57). ( $f$ ) Expression of HLA-A2 and TdT in leukemia cells detected in bone marrow of untreated and 1G4-treated mice at the time of sacrifice. Light gray histograms represent negative control. (g) BLI images of mice in T3-treated group on day 57. Two of the T3-treated mice died for unknown reasons on day 35 and day 55 after T cell therapy, following negative BLI and PB flow cytometry analysis 5 days earlier, respectively, indicating that death was not related to leukemia. (h) IFN- $\gamma$ levels in sera from untreated $(n=5), 1 \mathrm{G} 4(n=4)$ or T3 $(n=5)$ cell-treated mice on day 2 after T cell therapy, measured as mean fluorescence intensity (MFI) of IFN- $\gamma$ capturing fluorescent beads. Statistical analysis by one way ANOVA with Tukey's multiple comparison test. Box plots in (a) and (h) show interquartile range ( $25^{\text {th }}$ to $75^{\text {th }}$ percentile) with central bar indicating the median and whiskers indicating the range, while dots represent data from individual mice. 
a

b
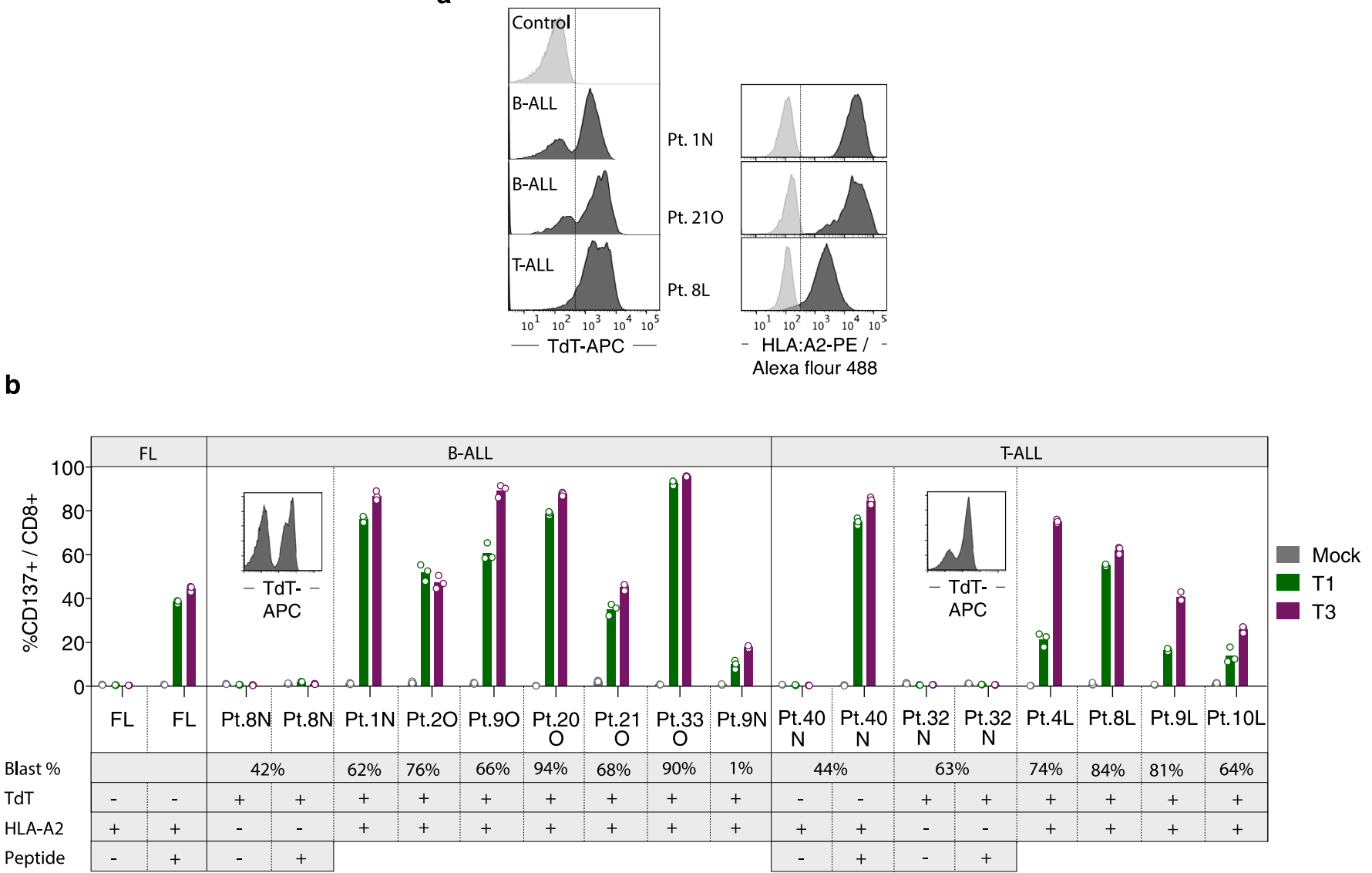

Extended Data Fig. 7 | Primary patient B-ALL and T-ALL cells express TdT and activate T1 and T3 cells. (a) TdT and HLA-A2 expression in diagnostic samples (PBMC) from three representative patients with B-ALL and T-ALL as analyzed by flow cytometry. Cytotoxicity data for these patients are shown in Fig. 4a, b. (b) Percentage of CD137+ events among T1, T3 and mock-transduced CD8 ${ }^{+}$cells after $18 \mathrm{~h}$ culture with primary B-ALL and T-ALL patient samples that were either HLA-A2 ${ }^{\text {pos }} T d T^{\text {neg }}$, HLA-A2 $2^{\text {neg }} T d T^{\text {pos }}$ or HLA-A2 ${ }^{\text {pos }} T T^{\text {pos }}$. Single positive patient samples were loaded or not with peptide-1 or -3 (concentration $10^{-7} \mathrm{M}$ ) for $1 \mathrm{~h}$ before co-culture with corresponding $\mathrm{T} 1$ or $\mathrm{T} 3$ cells. FL: Follicular lymphoma, included as an HLA-A2 ${ }^{\text {pos }} \mathrm{TdT} \mathrm{T}^{\text {neg }}$ control. Numbers below patient index show percentages of malignant blasts in the patient sample as analyzed by flow cytometry. Bar graphs in (b) show mean of 2-3 technical replicates run for individual patients. 
a

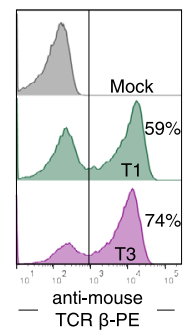

b

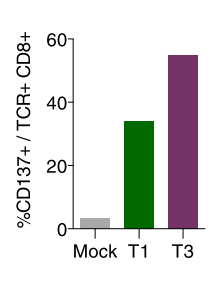

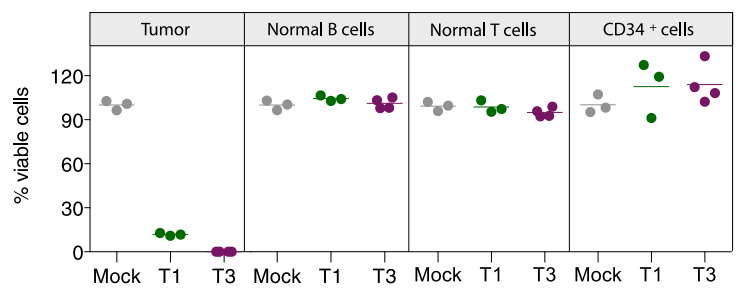

d

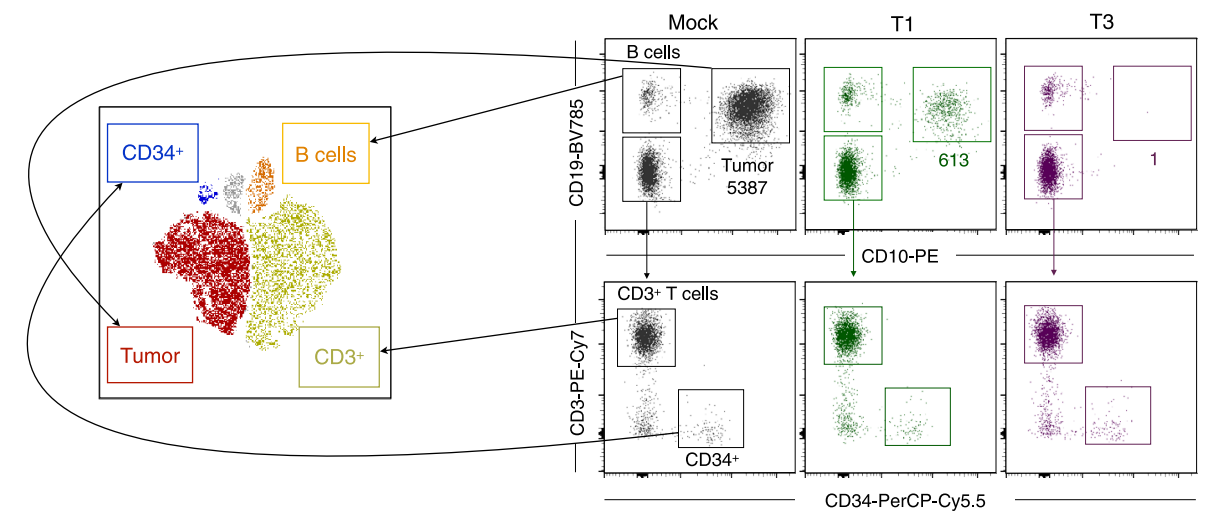

Extended Data Fig. 8 | Patient-derived CD8 ${ }^{+}$T cells transduced with T1 or T3 efficiently kill autologous B-ALL cells. (a) Transduction efficiency of the T1 (green) and T3 (purple) TCRs in normal CD8 ${ }^{+} \mathrm{T}$ cells from an HLA-A2 $2^{\text {pos }}$ TdT ${ }^{\text {pos }}$ patient diagnosed with B-ALL. Histograms are gated on live, CD8 ${ }^{+} \mathrm{T}$ cells. (b) T1 and T3 cell activation after $18 \mathrm{~h}$ co-culture with the autologous blasts. (c) Quantification of malignant cells, normal B, T and CD34+lin- progenitor cells after performing the flow cytometry-based cytotoxicity assay for $72 \mathrm{~h}$. Data points represents technical replicates (3-4) from one representative experiment out of 2 performed and horizontal lines show mean. (d) Flow cytometry dot plots from one of the test replicates in c, showing gating strategy to identify tumor blasts $\left(C D 19^{+} \mathrm{CD} 10^{+}\right)$, normal B cells $\left(\mathrm{CD} 19^{+} \mathrm{CD} 10^{-}\right)$, T cells $\left(\mathrm{CD} 19^{-} \mathrm{CD} 10^{-} \mathrm{CD} 3^{+}\right)$and $\mathrm{CD} 34^{+}$progenitor cells $\left(\mathrm{CD} 19^{-} \mathrm{CD} 10^{-} \mathrm{CD} 3^{-} \mathrm{CD} 34^{+}\right)$. Populations were gated and overlaid on a t-SNE plot with designated colors as indicated (left view). Inset numbers in d show event count of tumor cells after co-culture with mock, $\mathrm{T} 1$ and $\mathrm{T} 3$ cells. 
a
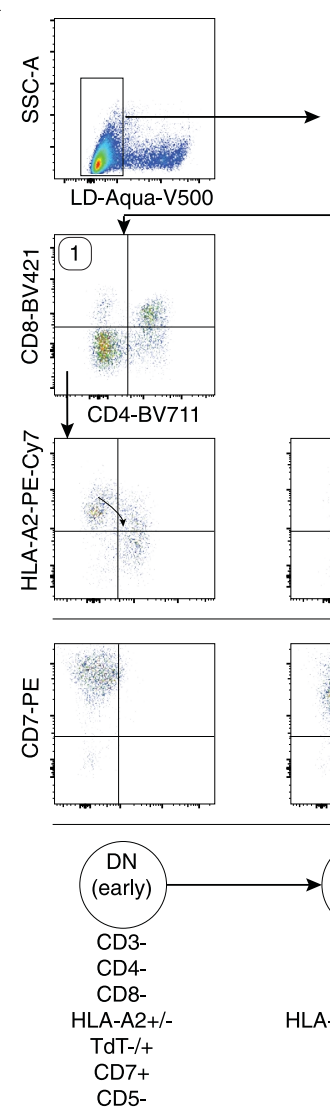
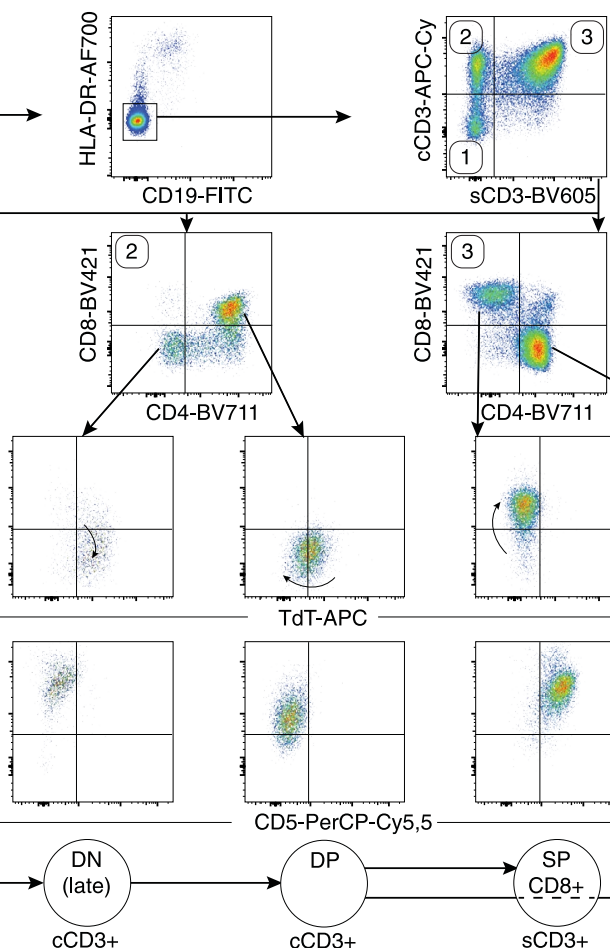

cCD3+

CD4-

HLA-A2 low/neg

$\mathrm{TdT}+$

CD7+

CD7+
CD5-
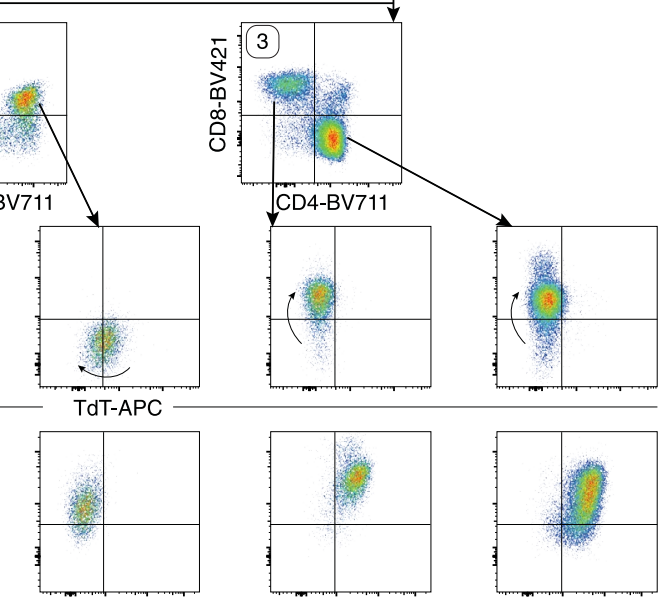
CD5-PerCP-Cy5,5
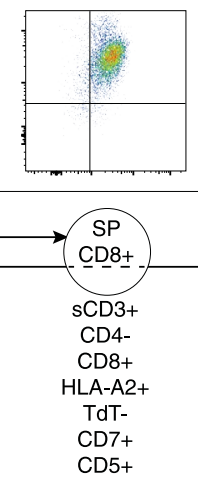
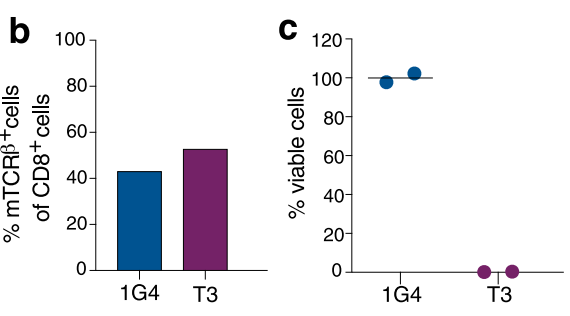

d
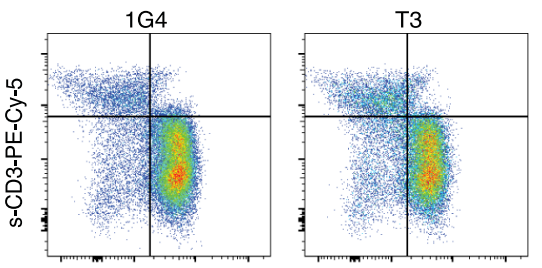

TdT-APC

e

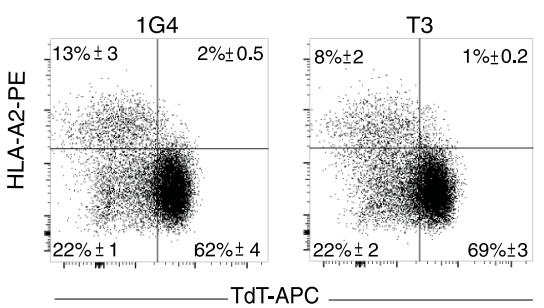

Extended Data Fig. 9 | Expression of TdT and HLA-A2 during T cell development. (a) Flow cytometric analysis of thymocytes from normal human thymus removed from a four months old child with congenital cardiac defect (but otherwise healthy) in conjunction with cardiac surgery. Flow plots show gating strategy to define four key developmental stages during thymocyte differentiation, including 1) early double negative (DN), 2) late double negative, 3) double positive (DP) and 4) single positive (SP, either $C D 4^{+}$or $C D 8^{+}$) thymocytes. Arrows within the FACS plots in third row indicate direction of differentiation with regard to expression of TdT and HLA-A2 during these developmental stages, illustrated with continuous lines in Fig. 6b. (b) Percentage of TCR-transduced cells among CD8 ${ }^{+} T$ cells isolated from spleens of three humanized NSG mice engrafted with healthy cord blood following transduction and expansion, prior to infusion into littermates day 0 , as illustrated in Fig. 6c. (c) Functionality of human TCR-transduced T cells shown in (b), as determined by the number of viable BV173 cells present after $72 \mathrm{~h}$ co-culture, in percent of corresponding numbers following treatment with $1 \mathrm{G} 4$ cells, quantified by flow cytometry (E/T ratio of 1/1). Co-cultures were started on the same day as T cells were injected into mice. Data points show 2 technical replicates from one experiment. (d,e) Representative FACS plots of viable single hCD45+CD19-CD33- thymocytes from 1G4 or T3-treated humanized NSG mice at terminal analysis on day 17 post T cell infusion illustrating surface CD3 and TdT expression (d) and HLA-A2 and TdT expression (e). Inset numbers show mean \pm s.e.m., $n=8$ per group. 


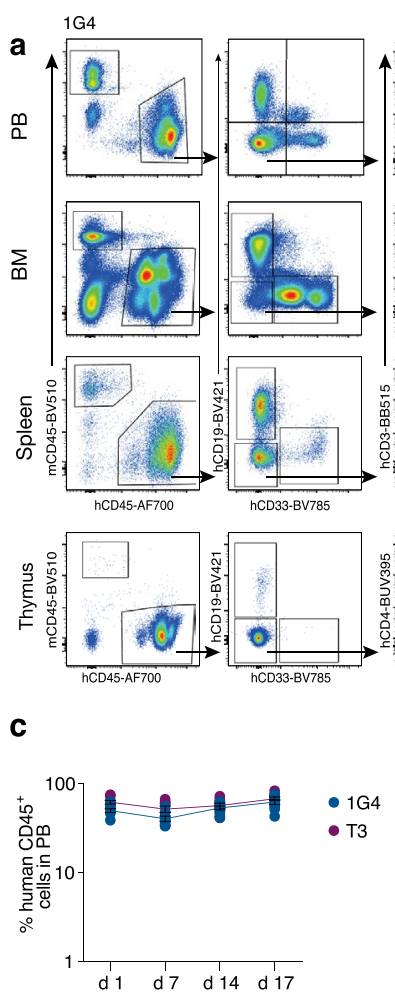

f

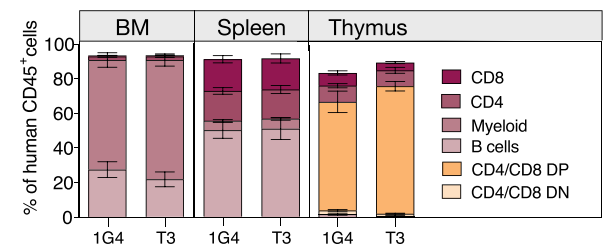

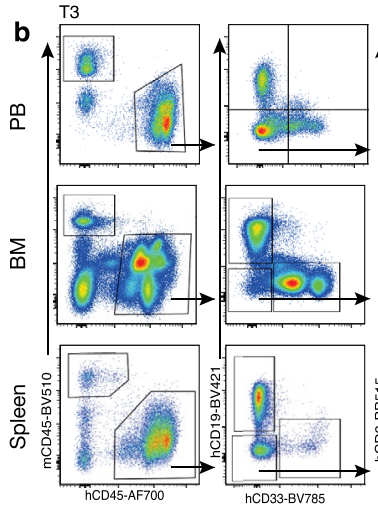
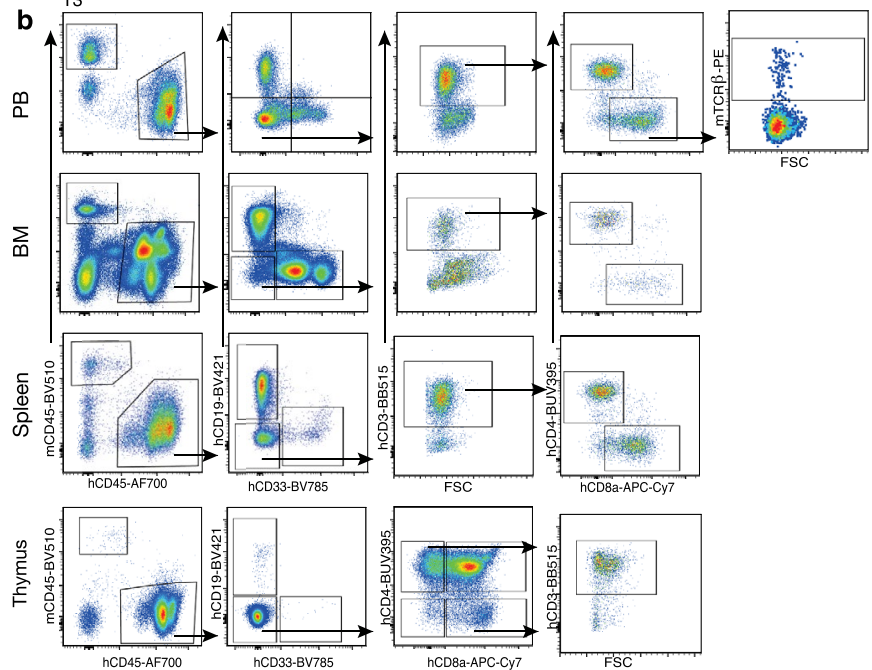

e

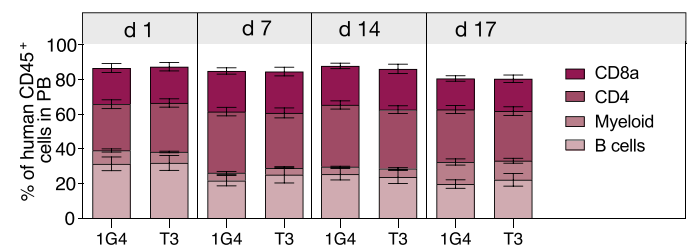

g

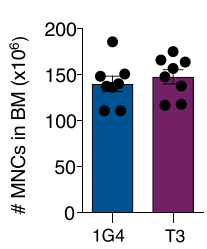

h

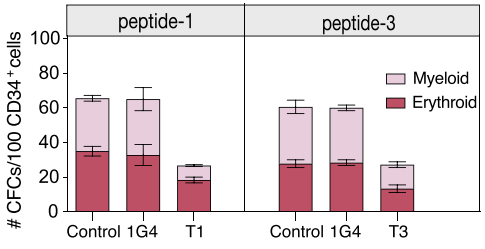

Extended Data Fig. 10 | TdT TCR-transduced cells do not impact normal hematopoiesis in vitro or in vivo. (a,b) Representative FACS plots from terminal analysis of viable single MNCs in PB, BM, spleen and thymus from humanized NSG mice treated with 1G4 (a) and T3 (b) cells, to determine the percentage of TCR-transduced T cells and lineage distribution (myeloid: $C D 33^{+}, \mathrm{B}$ cells: $\mathrm{CD} 19^{+}, \mathrm{T}$ cells: $\mathrm{CD} 3^{+} \mathrm{CD} 4^{+}$or $\left.\mathrm{CD} 3^{+} \mathrm{CD} 8^{+}\right)$. $(\mathrm{c}, \mathrm{d})$ Percentage human $\mathrm{CD} 45^{+}$cells (out of human and mouse $\mathrm{CD} 45^{+}$cells) in PB at indicated days after T cell infusion (c), and in BM, spleen and thymus of humanized NSG mice treated with $1 G 4$ or T3 cells at terminal analysis on day 17 (d). (e,f) Percentage lineage distribution within human CD45+ engrafted cells in PB at indicated days after T cell infusion (e) and in BM, spleen and thymus at terminal analysis (f). (g) BM cellularity at terminal analysis. $n=8$ per group in c-g. (h) Myeloid and erythroid colonies generated from sorted normal human BM CD34+Lineage ${ }^{-}$cells from one donor co-cultured without T cells (control), or with $1 \mathrm{G} 4, \mathrm{~T} 1$ or $\mathrm{T} 3$ cells for $48 \mathrm{~h}$ in the presence of peptide- 1 or peptide- 3 at an $\mathrm{E} / \mathrm{T}$ ratio of $2 / 1$ ( 2 technical replicates per group). Data are presented as mean \pm s.e.m. 


\section{Reporting Summary}

Nature Research wishes to improve the reproducibility of the work that we publish. This form provides structure for consistency and transparency in reporting. For further information on Nature Research policies, see our Editorial Policies and the Editorial Policy Checklist.

\section{Statistics}

For all statistical analyses, confirm that the following items are present in the figure legend, table legend, main text, or Methods section.

$\mathrm{n} / \mathrm{a}$ Confirmed

$\bigotimes$ The exact sample size $(n)$ for each experimental group/condition, given as a discrete number and unit of measurement

\ A statement on whether measurements were taken from distinct samples or whether the same sample was measured repeatedly

The statistical test(s) used AND whether they are one- or two-sided

Only common tests should be described solely by name; describe more complex techniques in the Methods section.

\ $\square$ A description of all covariates tested

\A description of any assumptions or corrections, such as tests of normality and adjustment for multiple comparisons

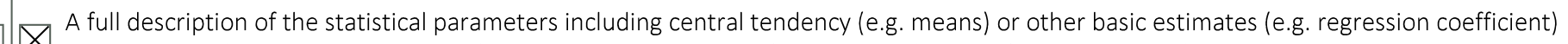

AND variation (e.g. standard deviation) or associated estimates of uncertainty (e.g. confidence intervals)

For null hypothesis testing, the test statistic (e.g. $F, t, r$ ) with confidence intervals, effect sizes, degrees of freedom and $P$ value noted

Give $P$ values as exact values whenever suitable.

Х $\square$ For Bayesian analysis, information on the choice of priors and Markov chain Monte Carlo settings

Х $\square$ For hierarchical and complex designs, identification of the appropriate level for tests and full reporting of outcomes

$\square$ Estimates of effect sizes (e.g. Cohen's d, Pearson's $r$ ), indicating how they were calculated

Our web collection on statistics for biologists contains articles on many of the points above.

\section{Software and code}

Policy information about availability of computer code

Data collection 1. Flow cytometry was performed on BD LSR II Cytometer (BD Biosciences) and data was acquired with the help of BD FACSDIVA V8.0.1 software.

2. Cell sorting was performed with SH800 (Sony Biotechnology), BD FACSAria II (BD Biosciences) or BD FACS AriaFusion (BD Biosciences) cell sorter.

3a. High performance liquid chromatography (HPLC) and mass spectrometry (MS) data was acquired by a system consisting of Agilent 1100 gradient HPLC system (Agilent) and an LTQ-FT Ultra mass spectrometer (Thermo Scientific).

3b. Immunoprecipitation- targeted Mass spectrometry (IP-MS) data was acquired using an Ultimate 3000 nano-UHPLC system (Dionex, Sunnyvale, CA, USA) connected to a Q Exactive mass spectrometer (ThermoElectron, Bremen, Germany) equipped with a nano electrospray ion source.

4. Bioluminescence imaging of mice was performed with IVIS Spectrum in vivo imaging system (PerkinElmer).

Data analysis $\quad$ 1. Flow cytometry data were analyzed with FlowJo versions 9 and 10.

2. Numerical data were statistically analyzed and graphs were generated with the help of GraphPad Prism versions 6,7 and 8 software. 3a. The tandem mass spectra were matched against the homo sapiens canonical (70,000 entries) database using the Mascot search engine (version 2.2.04, Matrix Science, London, UK, www.matrixscience.com). Post-analysis of the HPLC-MS data was performed with Proteome Discoverer version 2.2 (Thermo Electron), and then submitted to the Uniprot Homo sapiens database (71591 entries), using Mascot v. 2.2.07 (www.matrixscience.com) as described in the methods section.

3b. Raw data from targeted MS analysis were analyzed using the XcaliburTM software version 4.1.

4. Bioluminescence imaging was analyzed with Living image software version 4.5.2 (PerkinElmer)

5. Peptide-MHC complex models were visualized by the PyMOL molecular visualization system (version 2.3). Peptide (ILAKFLHTL) from human telomerase reverse transcriptase (PDB accession code: 5MEQ) and (FVLELEPEWTV) derived from Toxoplasma gondii (PDB accession code: 5D9S) were utilized to generate peptide- 1 and -3 HLA-A2 models respectively as described in the methods section.

6. Curated human proteome databases UniProtKB/Swiss-Prot and Protein Data Bank were queried by employing ScanProsite tool (https:// 
Policy information about availability of data

All manuscripts must include a data availability statement. This statement should provide the following information, where applicable:

- Accession codes, unique identifiers, or web links for publicly available datasets

- A list of figures that have associated raw data

- A description of any restrictions on data availability

The data that support the findings of this study are included in the manuscript and in supplementary information. Source data are also provided. Additionally, datasets used in the study are: Protein Data Bank (accession codes: 5MEQ, https://www.rcsb.org/structure/5MEQ and 5D9S, https://www.rcsb.org/structure/5D9S), Homo sapiens canonical database using the Mascot search engine (version 2.2.04, www.matrixscience.com), Uniprot Homo sapiens database, using Mascot v. 2.2.07, www.matrixscience.com), curated human proteome databases UniProtKB/Swiss-Prot and Protein Data Bank by ScanProsite tool (https://prosite.expasy.org/ scanprosite/), peptide-MHC class I binding prediction algorithm NetMHC version 4.0 (http://www.cbs.dtu.dk/services/NetMHC/)

\section{Field-specific reporting}

Please select the one below that is the best fit for your research. If you are not sure, read the appropriate sections before making your selection.

$\bigotimes$ Life sciences $\quad \square$ Behavioural \& social sciences $\quad \square$ Ecological, evolutionary \& environmental sciences

For a reference copy of the document with all sections, see nature.com/documents/nr-reporting-summary-flat.pdf

\section{Life sciences study design}

All studies must disclose on these points even when the disclosure is negative.

Sample size In experiments with xenograft B-ALL cell line models, we expected to see $25 \%$ decrease in the tumor bioluminescence signal in the treated animals compared to the control T-cell treated mice. In order to reach a p value of 0.05 with $95 \%$ power, we chose to have at least 7 mice per group. We used this as a guideline to choose numbers of mice per T cell treatment group (DMF5 or T3) in primary human B-ALL xenograft model (to observe therapeutic efficacy) and humanized mouse model engrafted with HLA-A2pos human cord blood cells (to observe lack of toxicity) without employing power calculations. Samples sized were expected to be sufficient based upon our previous observations with cell line based B-ALL models.

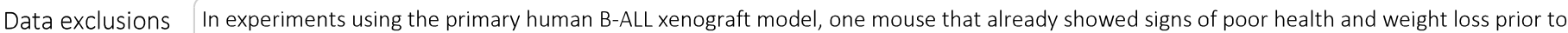
T-cell injections, had to be euthanized 2 days post treatment according to the guidelines from institutional animal experimentation ethics committee and excluded prior to any post-treatment analysis. For the experiments using the humanized mouse model engrafted with HLAA2pos human cord blood cells, one mouse was excluded due to the reconstitution failure of B cell lineage prior to T-cell treatment, according to the pre-determined exclusion criteria.

Replication $\quad$ Replication of experiments is described in detail in all figure legends. Briefly, survival and tumor bioluminescence imaging data from two independent experiments were pooled and shown in the manuscript. Two independent experiments were performed using the primary human B-ALL xenograft model with very similar results. One experiment was performed using the humanized mouse model engrafted with HLA-A2pos human cord blood cells.

ALL patient samples were tested at least twice to verify reproducibility and all technical replicates are shown for one representative experiment. The experiment shown in Fig. $2 \mathrm{~g}$ was performed once, but the functional analysis was performed with two independent methods with different readouts (i) ELISA (IFN-gamma secretion) and (ii) flow cytometric measurements of T cell activation by CD137 upregulation. Results from both T-cell functional readouts correlated strongly with each other, as shown in the manuscript in Extended Data Fig. 2c.

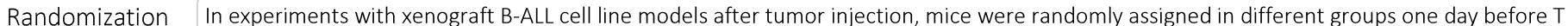
cell therapy. There was no difference in the tumor BLI signal between experimental groups before start of therapy (by one-way ANOVA with Tukey's multiple comparison test).

There is generally a relatively large variation in the tumor/human cell engraftment in the primary human B-ALL xenograft model and in the humanized mouse model engrafted with HLA-A2pos human cord blood cells. Therefore mice were assigned to different groups based upon their pre-treatment tumor burden or human cell engraftment in the bone marrow/blood to ensure that experimental groups had similar means prior to T-cell treatment. For the in vitro experiments, randomization was not performed as it is not applicable and is not generally performed in the field.

Blinding Colonies generated from sorted normal adult human BM CD34+ cells were counted blindly. The investigators were not blinded during group allocation or analysis in mice experiments. Blinding would be impractical due to the limitations related to manpower, and because of the potential alloreactivity that needed to be closely assessed. Due to the nature of the other in vitro experiments, blinding was not possible and is not generally performed in the field as data acquisition is quantitative (flow cytometry or MS) rather than qualitative, and therefore less influenced by observer bias. 


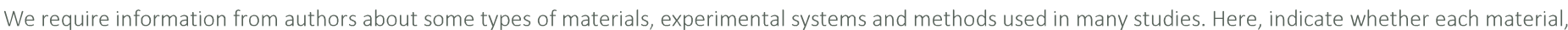

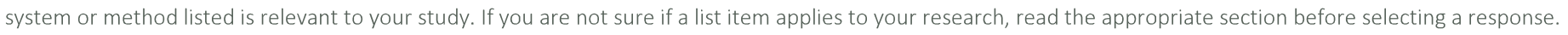

\begin{tabular}{|c|c|c|c|}
\hline \multicolumn{2}{|r|}{ Materials \& experimental systems } & \multicolumn{2}{|c|}{ Methods } \\
\hline $\mathrm{n} / \mathrm{a}$ & Involved in the study & $\mathrm{n} / \mathrm{a}$ & Involved in the study \\
\hline \begin{tabular}{|r} 
\\
\end{tabular} & $\bigotimes$ Antibodies & Х & $\square$ ChIP-seq \\
\hline - & $\bigotimes$ Eukaryotic cell lines & $\square$ & $\bigotimes$ Flow cytometry \\
\hline Х & $\square$ Palaeontology and archaeology & $\bigotimes$ & $\square$ MRI-based neuroimaging \\
\hline$\square$ & $\bigotimes$ Animals and other organisms & & \\
\hline - & \ Human research participants & & \\
\hline Х & $\square$ Clinical data & & \\
\hline$\square$ & $\bigotimes$ Dual use research of concern & & \\
\hline
\end{tabular}

\section{Antibodies}

Antibodies used The following fluorescently conjugated anti-human antibodies were acquired from BD Biosciences or BioLegend unless otherwise
specified: Anti-HLA-A2 (BB7.2), -CD62L (DREG-56), CD56 (HCD56, B159), -CD57 (HNK-1), -CD45RO (UCHL1), -CD45RA (HI100), -CCR7
(150503), -CD137 (4B4-1),-CD45 (HI30), -TdT (E17-1519), -CD10 (HI10a), -CD19 (HIB19, SJ25C1), -CD38 (HIT2), -CD34 (581, 8G12), -
CD1a (HI149), -CD2 (S5.2, RPA-2.10),-CD3 (UCHT1, OKT3, HIT3a), -CD8a (RPA-T8), -CD4 (RPA-T4), -CD5 (UCHT2, L17F12), -CD7 (M-
T701), -CD33 (WM-53), -CD11b (ICRF44),-CD14 (61D3), -CD20 (clone 2H7), -CD56 (B159), -CD235a,b (HIR2), -CD99 (DN16, Bio Rad), -
CD3 (SK7, eBioscience),-CD14 (HCD14, 61D3 eBioscience), anti-pan HLA class I antibody (W6/32). Anti-mouse antibodies: CD45 (30-
F11), TCRß chain (H57-597) and Ter119 (TER-119). Live/Dead Fixable Near-IR Dead Cell Stain kit (Life Technologies), 7AAD (Sigma
Aldrich) or DAPI (Thermofisher) were used to exclude dead cells in all flow cytometry experiments. The following antibodies for ELISA
were acquired from BD Pharmingen or R\&D Systems: mouse anti-human IFN- $y$ capture antibody (NIB42) and Biotin Mouse Anti-
Human IFN- $y$ detection antibody (4S.B3). Supplementary Table 5 lists all antibodies with supplier, species, catalogue number, clone
name, fluorochromes, validated research application and dilution factor used.

Validation

All antibodies used in the study are available commercially and have been validated by commercial suppliers for use in diagnostics/ research on samples generated from humans or mice, and for flow cytometry or ELISA as applicable. Detailed information is provided in Supplementary Table 5.

\section{Eukaryotic cell lines}

Policy information about cell lines

Cell line source(s)

The following cell lines were used in the study: NALM-6, BV173, EBV-LCL, HPB-ALL, T2, REH, RD, U-2 OS, FM-6, HeLa, HaCaT, COLO688, EA.hy926, U-87 MG, Daoy, HCT-116, CHP-212, MCF7, K562, RS4;11, Phoenix AMPHO. Cell lines were obtained from American Tissue Culture Collection (ATCC) and Deutsche Sammlung von Mikroorganismen und Zellkulturen (DSMZ). EBV-LCL cell lines were generated in-house by immortalizing human PBMC's from HLA-A2 positive and negative donors with Epstein-Barr viral supernatants. Epstein-Barr viral supernatants were produced in B95-8 cell line.

Authentication

Authenticated cell lines were purchased from ATCC and DSMZ (RS4;11, T2, RD, K562, EA.hy926, Daoy, HCT-116, CHP-212, Phoenix AMPHO), and cryopreserved aliquots labeled according to passage. Only low passages (1-4 passages) were used to start cultures. The identity of the passage used ( 5 or higher) experimentally of the cell lines NALM-6, BV173, REH, HPB-ALL, U-2 OS, FM-6, HeLa, HaCaT, MCF7, COLO 688, U-87 MG was ascertained by short tandem repeat DNA profiling, a service provided by Labcorp DNA Identification Lab, NC, USA (formerly Genetica, https://celllineauthentication.com/). In-house immortalized EBV-LCL cells were regularly tested for CD20 or CD19 staining to confirm their B cell origin.

Mycoplasma contamination

Cells were tested regularly for mycoplasma contamination, and were confirmed negative before experimental use.

Commonly misidentified lines (See $\underline{I C L A C}$ register)

HPB-ALL was used as a T-ALL cell line with desirable characteristics (TdT pos, HLA-A2 neg). Although registered in the ICLAC database as a commonly misidentified cell-line, we acquired an authenticated cell line from DSMZ stock (ACC-483). The ICLAC database also states that DSMZ has a correct version of non-contaminated cell line.

\section{Animals and other organisms}

Policy information about studies involving animals; ARRIVE guidelines recommended for reporting animal research

Laboratory animals

For establishing xenograft B-ALL cell line models, 8- to 10-weeks-old female and male NOD-scid IL2Rgnull (NSG) mice were used. To establish primary human B-ALL xenografted mice, 9-15 weeks old female NOD.Cg-Prkdcscid II $\mathrm{rgtm} 1 \mathrm{Wj} / \mathrm{SzJ}$ (NSG; Jackson Laboratory stock 005557) was used. Female NSG mice stably engrafted with HLA-A2pos human cord blood cells were purchased from the Jackson laboratory at 27 weeks of age (transplanted at 3 weeks of age).

Mice were housed in IVC-Mouse GM 500 cages with a light cycle of 4 a.m -4 p.m in $21^{\circ} \mathrm{C}$ with $50 \%$ humidity.

Wild animals

No wild animals were used.

Field-collected samples

No field-collected samples were used. 


\section{Human research participants}

Policy information about studies involving human research participants

Population characteristics

Recruitment

Ethics oversight
Patient demographics, disease characteristics and sample characteristics have been shown in the manuscript (Supplementary Tables 2, 3 and 4).

Pediatric and young adult relapsed/refractory (r/r) B-ALL patients were enrolled into and treated according to the Chimeric Antigen Receptor (CAR) T cell trials ClinicalTrials.gov Identifier NCT02435849 and NCT03123939; pediatric T-ALL patients according to NOPHO-ALL-2008 (NCT00816049). Institutional Review Board and ethical approvals to use primary human diagnostic blood and bone marrow samples, from pediatric and adult patients were obtained, as were informed written consent from patients or their guardians. Patient samples were selected for inclusion in our study after reviewing available diagnostic information from institutional biobanks. TdT and HLA-A2 status was determined as part of the diagnostic workup, and confirmed by flow cytometric measurements in our experiments. There was no self-selection bias. PB mononuclear cells (PBMCs) from healthy donor buffy coats were obtained from the blood bank of Oslo University Hospital, and PB or bone marrow mononuclear cells from leukemia patients were from biobanked, cryopreserved material (ethical approval numbers: REK 2018/879, REK 2018/1246).

Thymocytes were isolated from human thymus removed as a consequence of routine procedure for open cardiac surgery to correct a congenital cardiac defect (in an otherwise healthy child) following informed written consent from guardians and ethical approval (ethical approval number: REK 2019/31516). PB mononuclear cells (PBMCs) from healthy donor buffy coats were obtained from the blood bank of Oslo University Hospital.

Bone marrow mononuclear cells were obtained from four HLA-A2pos healthy donors collected at the Karolinska University Hospital with informed consent and ethical approval (EPN 2018/901-31).

This study was approved by the Regional Committee for Medical and Health Research Ethics (REC) South-East, Norway (2018/879, 2018/1246, 2019/31516), the Institutional Review Board and the Data Protection Officer, Oslo University Hospital, Swedish Ethical Review Authority, Stockholm (EPN 2018/901-31), and performed in accordance with the Declaration of Helsinki.

Note that full information on the approval of the study protocol must also be provided in the manuscript.

\section{Dual use research of concern}

Policy information about dual use research of concern

\section{Hazards}

Could the accidental, deliberate or reckless misuse of agents or technologies generated in the work, or the application of information presented in the manuscript, pose a threat to:

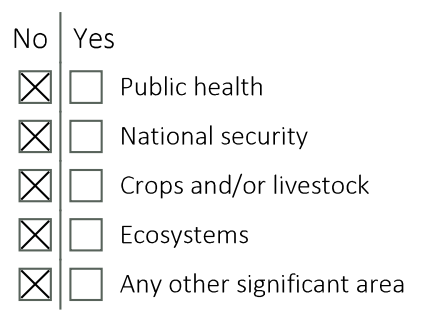

\section{Experiments of concern}

Does the work involve any of these experiments of concern:

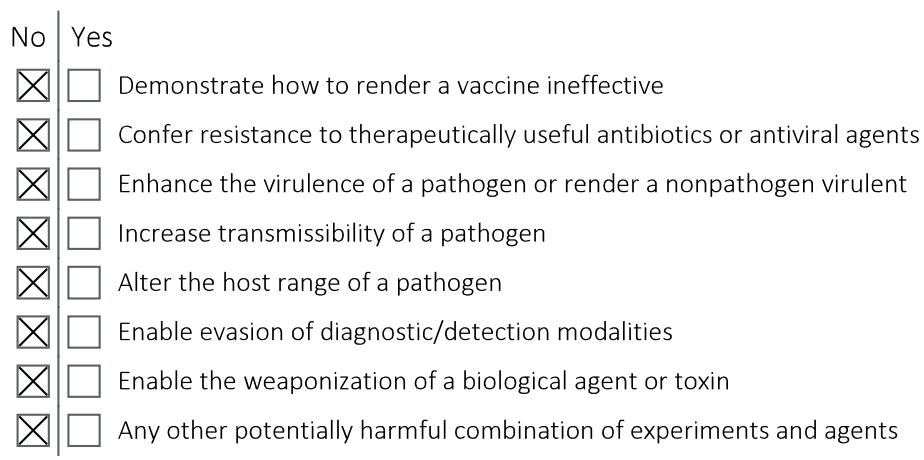


Plots

Confirm that:

\The axis labels state the marker and fluorochrome used (e.g. CD4-FITC).

The axis scales are clearly visible. Include numbers along axes only for bottom left plot of group (a 'group' is an analysis of identical markers).

$\square$ All plots are contour plots with outliers or pseudocolor plots.

\A numerical value for number of cells or percentage (with statistics) is provided.

\section{Methodology}

Sample preparation

Instrument

Software

Cell population abundance
Samples analyzed by flow cytometry contained mononuclear cells in suspension isolated from buffy coats by density gradient centrifugation that were cultured with standard media as detailed in the manuscript. Patient blood or bone marrow samples were harvested for diagnostic purposes and mononuclear cells were isolated by leukapheresis. Samples were then cryopreserved and stored in liquid nitrogen in designated cell biobanks in our institutions before use in the experiments as detailed in the methods. Cell lines utilized were cultured for variable amounts of time in recommended media prior to experiments.

Blood samples and bone marrow were harvested from murine xenograft B-ALL cell line models. RBC lysis was performed by ACK lysis buffer, followed by washing and surface or intracellular staining with different antibodies for flow cytometry analysis.

Peripheral blood, spleen, thymus and bone marrow was harvested from the primary human B-ALL xenograft model or humanized mouse model engrafted with HLA-A2pos human cord blood cells and prepared into single cell suspensions in PBS supplemented with fetal calf serum. All samples were incubated with Fc-Block prior to staining with monoclonal antibodies.

BD LSR II (BD Biosciences) equipped with high throughput sampler (HTS). Cell sorting was performed with SH800 (Sony Biotechnology), BD FACSAria II or BD FACS AriaFusion (BD Biosciences) cell sorter.

For data collection for all experiments, BD FACSDiva V8.0.1 was used. For data analysis FlowJo versions 9 and 10 were used.

Single cytotoxic T cells were sorted by FACS and expanded into T cell clones. Purity was confirmed with relevant peptide-MHC tetramer staining after ( $86 \%-100 \%$ purity). Tumor cell lines that were transduced with HLA-A2 or GFP and firefly luciferase, were purified by FACS (purity > 95\%). CD34+ cells used for CFC culture were sorted on a BD FACS AriaFusion (purity > 95\%). 
For pMHC multimer staining: From the live cell gate, CD8+ events were gated and subsequently, $\mathrm{pMHC}$ multimer+ events were identified as double positive for PE and APC conjugated pMHC multimers.

For flow cytometry-based cytotoxicity assays and T cell activation assays, tumor cell lines and transduced T cells were stained with surface antibodies and were gated as detailed in the methods and shown in Extended Data figures 4 and 8 . Primary patient samples were also stained with surface antibodies for the presence of tumor blasts, mature T/B cells or CD34+lincells. Effector cells in the same well were pre-labeled with CellTrace Violet (CTV, Life Technologies) for separation from live target cells. Tumor blasts, mature B and T cells, and CD34+lin- cells were gated and overlaid to be visualized as T-Distributed Stochastic Neighbor Embedding (tSNE) plots. CountBright Absolute Counting Beads were utilized to acquire equal numbers of events in each tested well.

To analyze presence of transduced T cells in blood and bone marrow of xenograft B-ALL cell line models, single live cells were gated as described in methods. Total leukocytes were defined as antigen positive for both human and mouse CD45. From CD45+ gate, human TCR-transduced T cells were identified as anti-human CD3+, CD8+ and anti-mouse TCR- $\beta+$. The murine constant part introduced into the TCR- $\beta$ chain served as a reporter for TCR transduction.

For the flow cytometric analysis of thymocytes, samples from normal human thymus removed from a four months old child with congenital cardiac defect were used. Flow plots displaying the gating strategy to identify the four key developmental stages during thymocyte differentiation (early double negative, late double negative, double positive and single positive) are shown in Extended data Fig. 9.

To generate the patient-derived xenograft model, viable T cell-depleted bone marrow cells from an HLA-A2pos B-ALL patient was identified through 7AAD and CD3 exclusion and yield sorted for transplantation. Upon termination, peripheral blood, bone marrow and spleen was subjected to detailed flow cytometry analysis using anti-human CD45, -CD8a, -CD4, -CD3, CD19, -HLA-A2, -CD10 and anti-mouse CD45, -Ter119 and -TCR $\beta$. The same panel was used to monitor the engraftment levels before and after $\mathrm{T}$ cell infusion.

To investigate the impact of T3 cells on normal human hematopoiesis in humanized NSG mice the persistence of infused T cells was monitored in PB, and impact of therapy on mature blood cell lineages was monitored in PB, spleen, thymus and bone marrow by flow cytometry with anti-human CD45, -CD8a, -CD19, -CD33, -CD4, -CD3 and anti-mouse CD45, -Ter119 and TCR $\beta$. The impact on human T cell progenitors in mouse thymus was investigated through surface and intracellular staining with anti-human TdT, -CD45, -CD8 -CD19, -CD3 (intracellular and surface), -CD4, -HLA-A2 and anti-mouse CD45.

Tick this box to confirm that a figure exemplifying the gating strategy is provided in the Supplementary Information. 